\title{
A guide to curating New World Melastomataceae collections with a linear generic sequence to world-wide Melastomataceae
}

\author{
Fabián A. Michelangeli ${ }^{1}$, Frank Almeda ${ }^{2}$, Renato Goldenberg ${ }^{3}$, Darin S. Penneys ${ }^{4}$ \\ ${ }^{1}$ Institute of Systematic Botany, New York Botanical Garden, 2900 Southern Blvd, Bronx, NY \\ 10458-5126 USA; https://orcid.org/0000-0001-7348-143X; fabian@nybg.org \\ ${ }^{2}$ California Academy of Sciences, Institute for Biodiversity Science and Sustainability, \\ Department of Botany, 55 Music Concourse Drive, Golden Gate Park, San Francisco, CA \\ 94118-4599, USA; https://orcid.org/0000-0001-5091-6875; falmeda@ calacademy.org \\ ${ }^{3}$ Departamento de Botânica, Universidade Federal do Paraná, R. Francisco H. dos Santos, s.n., \\ Campus do C. Politécnico, Curitiba 81531-980 BRAZIL; https://orcid.org/0000-0002-7047- \\ 6720; rgolden@ufpr.br
}

${ }^{4}$ University of North Carolina Wilmington, Department of Biology and Marine Biology, 601 S. College Rd., Wilmington, NC, 28403, USA; https://orcid.org/0000-0003-0727-2829; penneysd@uncw.edu

\begin{abstract}
The following guide is aimed at aiding in the curation of herbarium collections in Melastomataceae, with an emphasis on the New World species. It contains a summary of the taxonomic realignments at the tribal and generic level within Neotropical taxa of

Melastomataceae, as well as some general comments for other groups. A table with a generic linear sequence is also provided, as well as tables with new and synonymized New World genera since 2005 and all currently accepted species. Lastly, a table with the synonyms of over 1000 accepted Neotropical species that have been impacted by these generic realignments is also provided.
\end{abstract}

Resumen: La siguiente guía esta dirigida a ayudar la curaduría de las colecciones de herbario de Melastomataceae, con énfasis en las especies del Nuevo Mundo. Contiene un resumen de las realineamientos taxonómicos a nivel tribal y genérico en los taxones Neotropicales de Melastomataceae, así como comentarios generales de otros grupos. Se provee una tabla con una secuencia linear para los géneros, así como tablas con los géneros Neotropicales nuevos y sinonimizados desde 2005 y todas las especies aceptadas en este momento. Finalmente, también se incluye una tabla con los sinónimos de mas de 1000 especies que han sido afectadas por estos realineamientos genéricos.

\section{Introduction}

For most of the $20^{\text {th }}$ century Melastomataceae taxonomy relied on the tribal and generic concepts of Cogniaux's two main works, his treatment of the family for Flora Brasiliensis (Cogniaux 1885, 1886) and his global monograph for the Prodromus organized by the de Candolle dynasty (Cogniaux 1891). Cogniaux based many of his concepts (particularly the tribal ones) on Triana's 
global monograph (Triana 1871), which were in part formally made by Bentham (Bentham and Hooker 1867), and the latter was largely based on work by Triana that was presented at the world botanical congress (Triana 1866). Based on a morphological phylogenetic analysis, Renner (1993) proposed the first real overhaul of Cogniaux's system. However, shortly after, molecular phylogenetic analyses started to show that changes at both the tribal and generic level would have to be implemented as more data became available (Clausing et al. 2000, Clausing and Renner 2001b, Renner et al. 2001, Renner and Meyer 2001, Michelangeli et al. 2004, Renner 2004a, b).

During the $21^{\text {st }}$ Century, there has been a great advancement in our knowledge of Melastomataceae systematics, with both family-wide and more clade-specific phylogenies. Many of these changes have clarified the tribal placements of several genera, resulted in the recognition of new tribes, and changed the generic limits and concepts for several groups (details and citations below).

Unfortunately, translating these changes of Melastomataceae classification into curated herbarium collections is a challenge. Depending on the size of the herbarium, it can take a substantial effort to reorganize vouchers and folders. Another challenge is that in many cases when a plant family, or parts thereof, has been rearranged in publications, the new taxonomy and synonymy is not centralized. Curators are thus required to consult several sources (sometimes conflicting) in order to decide how to organize their collection. Some herbaria are organized alphabetically within family (e.g. NY, RB) and others use an evolutionary order based on monographs or phylogenetic hypotheses (e.g. BM, K, US). This latter approach was traditionally based on the linear sequences presented by the authors of large monographs, Cogniaux (1891) being the standard for Melastomataceae. This approach requires that the linear sequence used for curating collections is constantly updated to keep up with phylogenetic research. Finally, if a collection is partially or completely digitized, the new names all have to be entered in the database and the species determinations updated, or at least some indication of where the specimen is filed must be included.

We are in a point in time in which the tribal placement of almost every genus of Melastomataceae has been elucidated by molecular analyses, especially in the New World. This guide has been prepared as an aid for the curation and updating of Melastomataceae herbarium collections. As our knowledge of Old World Melastomataceae increases, we hope to update this guide with that knowledge. We present here a summary of the accepted genera in Melastomataceae worldwide in a linear sequence, a summary of the accepted species for the New World, and a table of traditionally accepted species names and their placement according to new generic realignments. References to generic realignments, checklists, floras, and monographs are also provided where appropriate.

\section{Methods}

The linear sequence provided here is based on a compilation of recent published and unpublished phylogenetic analyses (Clausing et al. 2000, Clausing and Renner 2001a, Clausing and Renner 2001b, Michelangeli et al. 2004, Bécquer-Granados et al. 2008, Martin et al. 2008, Amorim et al. 2009, Penneys et al. 2010, Michelangeli et al. 2011, Goldenberg et al. 2012, Michelangeli 2013, Penneys 2013, Penneys and Judd 2013a, Michelangeli et al. 2014, Goldenberg et al. 2015, Kriebel et al. 2015, Almeda et al. 2016, Reginato et al. 2016, Rocha et al. 2016a, Rocha et al. 2016b, Veranso-Libalah et al. 2017, Dellinger et al. 2018, Goldenberg et al. 2018, Rocha et al. 2018, Veranso-Libalah et al. 2018, Bacci et al. 2019, Bochorny et al. 2019, Guimarães et al. 
2019, Veranso-Libalah et al. 2019, Wurdack and Michelangeli 2019, Zhou et al. 2019a, Zhou et al. 2019b). The number of species per genus and comments on generic limits are based on the systematic and/or monographic studies cited below. Additionally, regional floras and checklists were consulted for determining numbers of accepted species in Neotropical groups (Macbride 1941, Alain 1957, Wurdack 1973, 1980, Howard 1989, Wurdack et al. 1993, Jørgensen and León-Yánez 1999, Liogier 2000, Almeda 2001, Berry et al. 2001, Leon 2006, Almeda et al. 2007a, Almeda et al. 2007b, Almeda 2009, Baumgratz et al. 2010, Cardoso et al. 2017, Ulloa Ulloa et al. 2017).

We provide references to published monographs in the last 40 years for Neotropical taxa and discuss which genera are currently assigned to the different tribes, but there is no explicit mention of the characters that support each group or genera within the tribes as it is not the scope of this guide.

\section{Results}

The Olisbeoideae is not currently divided into tribes. The number of currently recognized tribes in the Melastomatoideae is 18 (Table 1) with one resurrected tribe (Cyphostyleae) and six that have been proposed in the last 10 years: Cambessedesieae, Eriocnemeae, Henrietteeae, Lithobieae, Marcetieae, and Trioleneae. Additionally, there are a few genera that have been recovered in isolated positions in recent phylogenetic analyses that have no formal tribal placement as of this writing (see below).Dissochaeteae and Kibessieae are found exclusively in the Old World; Astronieae, Melastomateae, Olisbeoideae, and Sonerileae are pantropical; and all remaining tribes are New World endemics.

The total number of Melastomataceae genera is ca. 177 with ca. 5750 species (Table 1). This represent an increase of $13-15 \%$ in the number of species over those previously provided (Renner 1993, Christenhusz and Byng 2016). There are 88 genera currently recognized in the New World. Of these, six genera have been resurrected, and six newly described since 2014 (Table 2). A total of 36 genera recognized 15 years ago are now synonymized (Table 3 ). These changes in generic delimitations have resulted in the reclassification of over 1100 species, ca. $30 \%$ of all New World species (Table 4). There are currently ca. 3700 accepted neotropical melastome species (Table 5). CSV or excel versions of all these tables are also available upon request from the first author.

There are ca. 86 genera in the Old World comprising ca. 2000 species (Table 1). Generic delimitation problems, especially within the Sonerileae, prevent a clear estimate of the number of genera and species, and the lack of monographs and large regional floras hinders our estimates on species numbers.

The organization of the linear sequence presented in Table 1 follows the best current phylogenetic estimates of the Melastomataceae. The table is ordered first by a tribe number (and name), followed by a genus number. The genus numbers are in increments of 10 in order to accommodate any future generic realignments. For practical reasons, groups that contain both Old and New World genera have been divided first by geography and then organized based on either phylogenetic data or alphabetically when unavailable or problematic.

The following is a brief summary of the currently recognized tribes with notes on the number of genera, recent taxonomic changes, and sources of monographs and revisions. 


\section{SUBFAMILY OLISBEOIDEAE}

The subfamily Olisbeoideae, not formally divided into tribes (although some classifications recognize this group at the tribal rank as the Memecyleae), has four genera in the Old World and two in the New World (Stone 2005). Mouriri and Votomita, the two neotropical genera, were last monographed over 40 years ago (Morley 1976).

\section{SUBFAMILY MELASTOMATOIDEAE}

\section{Astronieae}

A tribe of four Old World genera (Penneys 2013) and one neotropical genus, Tessmannianthus, (Maurin et al. in prep.; Penneys et al. in prep.) that has not been monographed.

\section{Bertolonieae}

The tribe Bertolonieae historically comprised several genera in the New and Old World, but recent phylogenetic analyses have recovered Bertolonia in an isolated position, thus, the tribe is currently recognized as including only that genus (Bacci et al. 2019). Bertolonia was last revised just over 30 years ago (Baumgratz 1989), but since then the genus has almost doubled in size (Bacci et al. 2018).

\section{Blakeeae}

A strictly neotropical tribe with two genera, Blakea and Chalybea. Blakea and Topobea were found to be polyphyletic (Penneys and Judd, 2013a; Penneys and Judd, 2013b), thus Topobea was placed in synonymy.. Chalybea along with Huilaea were placed in Miconieae (Cogniaux 1891, Renner 1993), but both morphological and molecular data have shown that together they form a clade sister to Blakea (Penneys and Judd 2013a). Moreover, the distinction of the two genera as separate entities is not supported by morphological or molecular data (Morales and González 2005, Morales 2010), resulting in all species of Huilaea being transferred to Chalybea (Bernal et al. 2015). There are no published monographs for Blakea or Chalybea, although one is available for the latter as part of an unpublished thesis (Morales 2010).

\section{Cambessedesieae}

A strictly neotropical and recently described tribe that includes three genera: Cambessedesia, Huberia and Merianthera (Bochorny et al. 2019). Cambessedesia was traditionally placed in the Microliceae (Cogniaux 1891, Renner 1993), but seed morphology (Almeda and Martins 2001) and molecular data (Fritsch et al. 2004, Goldenberg et al. 2012) have shown that it did not belong in this group (Goldenberg et al. 2012). Huberia and Merianthera, along with Behuria and Dolichoura were placed in the Merianieae (Cogniaux 1891, Renner 1993). The latter two genera have been included within a larger Huberia (Bochorny et al. 2019). There is a monograph for Merianthera (Goldenberg et al. 2012), and a synopsis for Huberia sensu stricto (Baumgratz 2004). The two species formerly in Dolichoura were discussed by Goldenberg and Tavares (2007) and the monotypic Bisglaziovia by Baumgratz et al. (2004). Unpublished theses contain monographs for Behuria (Tavares 2005) and Cambessedesia (Martins 1984, Fidanza 2009).

\section{Cyphostyleae}

A recently resurrected and strictly Neotropical tribe with four genera endemic to the interAndean valleys from Colombia to Peru (Michelangeli et al. 2011). Alloneuron was revised by 
Wallnöfer (1996) with some species added later (Wallnöfer 1999, Michelangeli and Ulloa Ulloa 2016). Wallnöfer (1996) also segregated some species into the newly described Wurdastom, which has now been monographed (Mendoza-Cifuentes 2020). A key to the species of Allomaieta (including Cyphostyla) is available (Lozano and Becerra-de-Lozano 1999). Quipuanthus, a monotypic herbaceous genus from Ecuador and Peru was recently described (Michelangeli et al. 2014).

\section{Dissochaeteae}

A tribe restricted to the Old World mainly of vines and climbers, currently undergoing both tribal and generic recircumscriptions (Kartonegoro et al. submitted). Renner (1993) had a broader concept and suggested that it should be grouped within the Miconieae based on the berry fruit type. However, early molecular systematics studies suggested that it may be more closely related or even embedded within the mostly Paleotropical Sonerileae (Clausing and Renner 2001b, Clausing and Renner 2001a). More recent analyses with increased taxon and loci sampling have placed it outside the Sonerileae (Zhou et al. 2019a). Kartonegoro et al. (submitted) provides a well-supported molecular phylogeny of the tribe supporting the recognition and circumscription of six genera of Dissochaeteae. Recent monographs are available for Creochiton (Kartonegoro and Veldkamp 2013), Dissochaeta (Kartonegoro et al. 2018) and Macrolenes (Kartonegoro et al. 2019).

\section{Eriocnemeae}

A recently described tribe with three genera, two of them monotypic (Eriocnema and Ochthephilus) (Penneys et al. 2020). The third genus, Physeterostemon, was only relatively recently described (Goldenberg and Amorim 2006)

\section{Henrietteeae}

A recently described neotropical tribe that includes genera formerly included in the Miconieae (Penneys et al. 2010). Currently three genera are recognized, with Loreya and Myriaspora synonymized under Bellucia, and Henriettella and Llewellynia under Henriettea. Kirkbridea is assigned to the tribe based on morphology alone. Bellucia and Loreya were monographed by Renner (Renner 1989).

\section{Kibessieae}

A strictly paleotropical tribe composed of a single genus, Pternandra. At times it has been placed within the Astronieae (Triana 1871, Cogniaux 1891) or it has placed in its own subfamily Kibessiodeae (Renner 1993). However, these two groups form separate, non-sister clades (Clausing and Renner 2001b, Bacci et al. 2019). Pternandra was last revised by Maxwell (1981).

\section{Lithobieae}

A monotypic tribe recently described to accommodate one herbaceous species of the Brazilian campos rupestres (Penneys et al. 2020).

\section{Marcetieae}

A strictly neotropical and recently described tribe that includes some 20 genera of mostly herbaceous South American species formerly in the Melastomateae and one genus formerly in 
the Microlicieae (Rocha et al. 2016a, Rocha et al. 2018). Several generic realignments, resurrections, and descriptions have occurred across of the tribe (Almeda et al. 2016, Rocha et al. 2018). Monotypic Mallophyton is assigned here based on morphology. Recent monographs exist for Aciotis (Freire-Fierro 2002), Macairea (Renner 1989), Sandemania (Renner 1987) and Siphanthera (Almeda and Robinson 2011). There are unpublished revisions for Acisanthera (Kriebel 2008), Comolia (Seco 2006), and Marcetia (Martins 1989).

\section{Melastomateae}

A group with world-wide distribution in tropical and subtropical environments including 45 genera. The limits of the tribe correspond largely with those of the Melastomateae sensu Renner (1993) with the exclusion of the genera now in Marcetieae and other minor modifications (Michelangeli 2013, Rocha et al. 2016a, Veranso-Libalah et al. 2017). The circumscription the African genera has recently been addressed (Veranso-Libalah et al. 2017, Veranso-Libalah et al. 2019, Veranso-Libalah et al. 2020). Within Neotropical Melastomateae the large genus Tibouchina has been recently segregated into four smaller genera, Andeasanthus, Chaetogastra, Pleroma, and Tibouchina (Guimarães et al. 2019), with Pleroma also including Itatiaia, Microlepis, Svitramia, and Tibouchinopsis (Da Silva et al. 2014, Romero and Alves Versiane 2014, Guimarães et al. 2019). Monographs for neotropical genera are available for Andesanthus (as Tibouchina sect. Lepidotae) (Todzia and Almeda 1991), Centradenia (Almeda 1977), Chaetolepis (Grimm and Almeda 2013), Pilocosta (Almeda and Whiffin 1980, Almeda 1993a), Pterolepis (Renner 1994a), and Pterogastra (Renner 1994b). An unpublished thesis contains a revision of Heterocentron (Whiffin 1972), and a checklist for the accepted names in Monochaetum was recently published (Alvear and Almeda 2019). Wurdack (1953) monographed Brachyotum, but the genus needs a new revision because several species have been described since then.

\section{Merianieae}

A strictly Neotropical tribe with six capsular-fruited genera. Compared to Renner's circumscription (Renner, 1993), it now contains genera formerly in the Bertolonieae (Macrocentrum and Salpinga) and excludes some genera now recovered in the Sonerileae or Cambessedesieae (Goldenberg et al. 2008, Cotton et al. 2014, Dellinger et al. 2018, Bacci et al. 2019, Bochorny et al. 2019). Issues persist on the systematics of every genus in the tribe (Mendoza-Cifuentes and Fernandez-Alonso 2010, 2012, Dellinger et al. 2018), thus the current taxonomy will surely change in the near future. The only genus that has been recently monographed is Axinaea (Cotton et al. 2014) and Adelobotrys was the subject of a detailed morphological analysis (Schulman and Hyvonen 2003).

\section{Miconieae}

A strictly neotropical tribe of berry-fruited plants. Its circumscription is largely that of Triana (1866, 1871) and Cogniaux (1891), but excluding some taxa currently transferred to Blakeeae and Henrietteeae (see above) (Penneys 2007, Penneys et al. 2010, Penneys and Judd 2013a, Penneys and Judd 2013b).

Recent work has reduced the number of genera in the Miconieae from ca. 25, to just one. Phylogenetic analyses have shown that the taxonomy of Miconieae was in desperate need of an overhaul (Michelangeli et al. 2004, Bécquer-Granados et al. 2008, Goldenberg et al. 2008, Martin et al. 2008, Michelangeli et al. 2008, Gamba and Almeda 2014, Kriebel et al. 2015, 
Reginato and Michelangeli 2016, Goldenberg et al. 2018). Miconia has been found to be paraphyletic with all other genera nested within it. Moreover, of the remaining genera with more than three species, only Charianthus, Maieta, and Mecranium have been recovered as monophyletic. This has led to the recognition of a single genus within the Miconieae, Miconia (Ionta et al. 2012, Majure and Judd 2013, Michelangeli and Meier 2013, Gamba and Almeda 2014, Michelangeli 2014, Michelangeli et al. 2016, Michelangeli and Goldenberg 2016, Skean et al. 2018, Michelangeli et al. 2019).

Recent monographs include taxa formerly in Charianthus (Penneys 2001), Conostegia (Kriebel 2016), Leandra section Leandra (Reginato 2016), Mecranium (Skean 1993), Pachyanthus sensu stricto (Bécquer Granados 2012), Tococa (Michelangeli 2005), Miconia section Chaneopleura for the Greater Antilles (Judd 2007), the Octopleura clade (mostly of species in formerly in Ossaea and Miconia) (Gamba and Almeda 2014), and a few clades endemic to the Antilles of species in Clidemia, Leandra, Miconia, Ossaea, Sagraea, and the endemic genera Calycogonium, Pachyanthus, and Tetrazygia (Judd and Ionta 2013, Judd et al. 2014a, Judd et al. 2014b, Majure et al. 2015a, Majure et al. 2015b, Judd et al. 2018a, Judd et al. 2018b). Unpublished theses contain monographs for three different groups of Miconia, mostly from Eastern Brazil (Goldenberg 2000, Caddah 2013, Meirelles 2015). Added together these monographs represent less than $17 \%$ of the species in the tribe underscoring the need for further taxonomic work.

\section{Microlicieae}

A strictly neotropical tribe of six genera, mostly from open areas in Brazil (Fritsch et al. 2004). The tribal concept was larger in the past (Cogniaux 1891, Renner 1993), but based on seed and anther morphology, and later on molecular data, the size of the tribe has been dramatically reduced (Fritsch et al. 2004). Poteranthera was assigned to the Melastomateae by Renner (1993) but it had been placed in the Microlicieae by all previous classifications of that tribe. Its placement in the Microlicieae has recently been corroborated by both molecular and morphological data (Rocha et al. 2016b). Revisions exist for Chaetostoma (Koschnitzke and Martins 2006), Rhynchanthera (Renner 1990), and Lavoisiera (Martins and Almeda 2017). There is a synopsis for Poteranthera (Kriebel 2012, Almeda and Pacifico 2018), and there is an unpublished revision for Trembleya (Martins 1997). That leaves the ditypic Stenodon, and the large and complex Microlicia, as the only groups not revised.

\section{Rhexieae}

A new world tribe of three genera of small shrubs and herbs. Two of the genera (Arthrostemma and Pachyloma) had been placed in Melastomateae (Renner 1993), but molecular and seed morphology place it with Rhexia (Michelangeli 2013, Rocha et al. 2016a, Guimarães et al. 2019). Arthrostemma does not have a formal revision, but all four species occur in Mesoamerica, thus the treatment for the family of this area serves as a revision (Almeda 2009). Rhexia is the only group in the family more diverse in North America than Central and South America and it was last revised over 50 years ago (Kral and Bostick 1969) with a recent phylogenetic analysis demonstrating the hybrid origin of some of the species (Ionta et al. 2007).

\section{Sonerileae}

A predominantly Old World tribe, with some Neotropical species recently recovered within this clade (Bacci et al. 2019, Wurdack and Michelangeli 2019). The group has a complicated 
taxonomic history with several small genera segregated and combined through the years (Cellinese 1997, Cellinese 1998). Recent phylogenetic analyses have shown that most of the currently recognized Old World genera are not monophyletic and the tribe is in dire need of a new taxonomic arrangement (Cellinese 1998, Kokubugata et al. 2019, Zhou et al. 2019a, Zhou et al. 2019b). In light of these issues we have opted for separating the genera geographically and then order them alphabetically until the taxonomy is updated. We have also included some genera that have been synonymized when we suspect they could be resurrected (in this case the number of species in Table 1 is blank). Neblinanthera and Tateanthus are included here in the Sonerileae based on unpublished molecular and morphological data (Penneys et al. in prep).

\section{Trioleneae}

A strictly neotropical and recently described tribe that includes two genera: Monolena and Triolena (Bacci et al. 2020). These genera were considered as part of the Bertolonieae based on fruit morphology (Cogniaux 1891, Renner 1993), but it seems that these are convergent characters (Bacci et al. 2020). As currently circumscribed, Triolena also includes Diolena and Diplarpea (Wurdack 1977, Bernal et al. 2015). There is a monograph for Monolena (Warner 2002) in Central America.

\section{Unplaced Taxa}

Rupestrea a genus of two species from Eastern Brazil has been recovered in an isolated position sister to a large clade containing all the groups with capsular fruits and developed pedoconnectives (Goldenberg et al. 2015). Centradeniastrum and Stanmarkia, two genera with two species each from Andean South America and northern Mesoamerica, respectively, have been recovered forming an isolated clade (Penneys et al. in prep.), but more data is necessary to ascertain their exact phylogenetic position within the family. For details on Centradeniastrum see Almeda (1997), and for Stanmarkia Almeda (1993b).

The African genera Dinophora and Phaeoneuron (which had been merged with the Asian Ochthocharis) have been recovered forming a clade sister to the exclusively Neotropical Marcetieae (Veranso-Libalah et al. 2018). Based on geography and morphology it is unlikely that these genera will be considered as part of the Marcetieae and their taxonomic status still needs clarification.

The monotypic Feliciadamia is an African genus of uncertain affinities. It was placed in the Sonerileae by Renner (1993), but Jacques-Félix (1994) proposed a new tribe "Feliciadamieae" for it. However, this name has not been validly published. Given its unusual morphology, it probably does not belong in the Sonerileae and it is included here as unplaced.

\section{Acknowledgements}

We wish to thank Ricardo Pacifico for his review of the list of Microlicia and Trembleya. This research was supported by the National Science Foundation DEB-0515665, DEB-0818399 DEB-1146409, DEB-1343612. We also thank the Plant and Fungal Tree of Life Project (PAFTOL) at the Royal Botanic Gardens, Kew, for access to unpublished data. 


\section{Literature Cited}

Alain, H. 1957. Flora de Cuba. Part. 3, Melastomataceae. Pages 9-62 in H. León, and Alain, Hmo, editor. Contribuciones ocasionales del Museo de Historia Natural del Colegio "De La Salle".

Almeda, F. 1977. Systematics of the Neotropical genus Centradenia (Melastomataceae). Journal of the Arnold Arboretum 58:73-108.

Almeda, F. 1993a. Pilocosta (Melastomataceae), revisited: A new species, polyploidy, and the base chromosome number of the genus. Novon 3:311-316.

Almeda, F. 1993b. Stanmarkia, new genus, of Melastomataceae from the volcanic highlands of western Guatemala and adjacent Mexico. Brittonia 45:187-203.

Almeda, F. 1997. Systematics of the Andean genus Centradeniastrum (Melastomataceae). BioLlania Ed. Esp. 6:153-166.

Almeda, F. 2001. Melastomataceae. Pages 1339-1419 in W. D. Stevens, C. Ulloa Ulloa, A. Pool, and O. M. Montiel, editors. Flora de Nicaragua. Missouri Botanical Garden Press, Saint Louis, Missouri.

Almeda, F. 2009. Melastomataceae. Pages 164-338 in G. Davidse, M. Sousa S, S. Knapp, and F. Chiang, editors. Flora Mesoamericana. Universidad Nacional Autonoma de Mexico, Mexico City.

Almeda, F., P. E. Berry, A. Freire-Fierro, A. Gröger, B. K. Holst, N. G. Luckana, F. A. Michelangeli, T. Morley, D. Penney, S. S. Renner, O. R. Robinson, J. J. Wurdack†, and K. Yatskievych. 2007a. Melastomataceae. Pages 397-417 in V. Funk, T. Hollowell, P. Berry, C. Kelloff, and S. N. Alexander, editors. Checklist of the Plants of the Guiana Shield (Venezuela: Amazonas, Bolivar, Delta Amacuro; Guyana, Surinam, French Guiana). Contrib. U.S. Natl. Herb. Washington, DC.

Almeda, F., R. Kriebel, and G. Umaña. 2007b. Melastomataceae. Pages 395-574 in B. E. Hammel, M. H. Grayum, C. Herrera, and N. Zamora, editors. Manual de Plantas de Costa Rica. Vol. VI. Dicotiledóneas - Haloragaceae - Phytolaccaceae. Missouri Botanical Garden Press, Saint Louis, MO.

Almeda, F., and A. B. Martins. 2001. New combinations and new names in some Brazilian Microlicieae (Melastomataceae), with notes on the delimitation of Lavoisiera, Microlicia, and Trembleya. Novon 11:1-7.

Almeda, F., F. A. Michelangeli, and P. L. Viana. 2016. Brasilianthus (Melastomataceae), a new monotypic genus endemic to ironstone outcrops in the Brazilian Amazon. Phytotaxa 273:269-282.

Almeda, F., and R. Pacifico. 2018. Neotropical Poteranthera (Melastomataceae: Microlicieae) Revisited. Systematic Botany 43:552-556.

Almeda, F., and O. R. Robinson. 2011. Systematics and Phylogeny of Siphanthera (Melastomataceae). Systematic Botany Monographs 93:1-101.

Almeda, F., and T. Whiffin. 1980. Pilocosta, a new genus of tropical American Melastomataceae. Systematic Botany 5:294-311.

Alvear, D. M., and F. Almeda. 2019. Revision of Monochaetum (Melastomataceae: Melastomateae) in Colombia. Systematic Botany Monographs 109:1-155.

Amorim, A. M., R. Goldenberg, and F. A. Michelangeli. 2009. A new species of Physeterostemon (Melastomataceae) from Bahia, Brazil, with notes on the phylogeny of the genus Systematic Botany 34:324-329. 
Bacci, L. F., A. M. Amorim, F. A. Michelangeli, and R. Goldenberg. 2018. Increased sampling in under-collected areas sheds new light on the diversity and distribution of Bertolonia, an Atlantic Forest endemic genus. Systematic Botany 43:767-792.

Bacci, L. F., A. M. Amorim, F. A. Michelangeli, and R. Goldenberg. 2020. Flower morphology is correlated with distribution and phylogeny in Bertolonia (Melastomataceae), an herbaceous genus endemic to the Atlantic Forest. Molecular Phylogenetics and Evolution 149:106844.

Bacci, L. F., F. A. Michelangeli, and R. Goldenberg. 2019. Revisiting the classification of Melastomataceae: implications for habit and fruit evolution. Botanical Journal of the Linnean Society 190:1-24.

Baumgratz, J. F. A. 1989. O genero Bertolonia Raddi (Melastomataceae): Revisão taxonômica e considerações anatômicas. Arquivos do Jardim Botanico do Rio de Janeiro 30:69-213.

Baumgratz, J. F. A. 2004. Sinopse de Huberia DC. (Melastomataceae: Merianieae). Revista Brasileira de Botânica 27:545-561.

Baumgratz, J. F. A., M. L. D’El Rei Souza, and R. D. M. Tavares. 2004. Bisglaziovia Cogn. (Merianieae, Melastomataceae): Considerações Taxonômicas e Biogeográficas. Bradea 10:75-80.

Baumgratz, J. F. A., K. Fidanza, R. Bernardo, B. Chiavegatto, R. Goldenberg, P. J. F. Guimarães, Ricardo Kriebel, A. B. Martins, Fabián A. Michelangeli, M. Reginato, R. Romero, M. L. D. E. R. Souza, and E. Woodgyer. 2010. Melastomataceae. Pages 12361277 Catálogo de plantas e fungos de Brasil.

Bécquer Granados, E. R. 2012. Taxonomía de Pachyanthus (Melastomataceae: Miconieae). Brittonia 64:179-207.

Bécquer-Granados, E. R., K. M. Neubig, W. S. Judd, F. A. Michelangeli, J. R. Abbott, and D. S. Penneys. 2008. Preliminary molecular phylogenetic studies in Pachyanthus (Miconieae, Melastomataceae). Botanical Review 74:37-52.

Bentham, G., and J. D. Hooker. 1867. Genera plantarum. Vol. 1. Reeve, Williams \& Norgate, London.

Bernal, R., S. R. Gradstein, and M. Celis. 2015. New names and new combinations for the Catalogue of the Plants and Lichens of Colombia. Phytoneuron 22:1-6.

Berry, P. E., A. Groger, B. K. Holst, T. Morley, F. A. Michelangeli, N. G. Luckana, F. Almeda, S. S. Renner, A. Freire-Fierro, O. R. Robinson, and K. Yatskievych. 2001. Melastomataceae. Pages 263-528 in P. E. Berry, B. K. Holst, and K. Yatskievych, editors. Flora of the Venezuelan Guayana. Missouri Botanical Garden Press, Saint Louis.

Bochorny, T., F. A. Michelangeli, F. Almeda, and R. Goldenberg. 2019. Phylogenetics, morphology and circumscription of Cambessedesieae: a new Neotropical tribe of Melastomataceae. Botanical Journal of the Linnean Society 190:281-302.

Caddah, M. K. 2013. Estudos taxonômicos e filogenéticos em Miconia sect. Discolor (Melastomataceae, Miconieae). UNICAMP.

Cardoso, D., T. Särkinen, S. Alexander, A. M. Amorim, V. Bittrich, M. Celis, D. C. Daly, P. Fiaschi, V. A. Funk, and L. L. Giacomin. 2017. Amazon plant diversity revealed by a taxonomically verified species list. Proceedings of the National Academy of Sciences 114:10695-10700.

Cellinese, N. 1997. Notes on the systematics and biogeography of the Sonerila generic alliance (Melastomataceae) with special focus on fruit characters. Tropical Biodiversity 4:83-93. 
Cellinese, N. 1998. Systematics and phylogeny of Phyllagathis and the Asian Sonerileae (Melastomataceae). American Journal of Botany 85:119.

Christenhusz, M. J. M., and J. W. Byng. 2016. The number of known plants species in the world and its annual increase. Phytotaxa 261:201-217.

Clausing, G., K. Meyer, and S. S. Renner. 2000. Correlations among fruit traits and evolution of different fruit within the Melastomataceae. Botanical Journal of the Linnean Society 133:303-326.

Clausing, G., and S. S. Renner. 2001a. Evolution of growth form in epiphytic Dissochateae (Melastomataceae). Organisms Diversity and Evolution 1:45-60.

Clausing, G., and S. S. Renner. 2001b. Molecular phylogenetics of Melastomataceae and Memecylaceae: Implications for character evolution. American Journal of Botany 88:486-498.

Cogniaux, C. A. 1885. Melastomaceae. Pages 1-510 in C. F. P. d. Martius, editor. Flora Brasiliensis. Fleischer, Leipzig.

Cogniaux, C. A. 1886. Melastomaceae. Pages 1-655 in C. F. P. d. Martius, editor. Flora Brasiliensis. Leipzig, Fleischer.

Cogniaux, C. A. 1891. Melastomaceae. G. Masson, Paris.

Cotton, E., F. Borchsenius, and H. Balslev. 2014. A revision of Axinaea (Melastomataceae). Scientia Danica. Series B, Biologica 4:1-120.

Da Silva, M. F. O., P. J. F. Guimaraes, and F. A. Michelangeli. 2014. Nomenclatural and taxonomic novelties in the tribe Melastomeae (Melastomataceae). Phytotaxa 186:222228.

Dellinger, A. S., M. Chartier, D. Fernández-Fernández, D. S. Penneys, M. Alvear, F. Almeda, F. A. Michelangeli, Y. Staedler, W. S. Armbruster, and J. Schonenberger. 2018. Beyond buzz-pollination - departures from an adaptive plateau lead to new pollination syndromes. New Phytologist 221:1136-1148.

Fidanza, K. 2009. Estudos taxonômicos em Cambessedesia DC. (Melastomataceae). Ph. D. Universidade Estadual de Campinas, Campinas.

Freire-Fierro, A. 2002. Monograph of Aciotis (Melastomataceae). Systematic Botany Monographs 62:1-99.

Fritsch, P. W., F. Almeda, S. S. Renner, A. B. Martins, and B. C. Cruz. 2004. Phylogeny and circumscription of the near-endemic Brazilian tribe Microlicieae (Melastomataceae). American Journal of Botany 91:1105-1114.

Gamba, D., and F. Almeda. 2014. Systematics of the Octopleura clade of Miconia (Melastomataceae: Miconieae) in Tropical America. Phytotaxa 179:1-174.

Goldenberg, R. 2000. O Gênero Miconia Ruiz \& Pav. (Melastomataceae): I. Listagens analíticas, II. Revisão Taxonômica da Seção Hypoxanthus (Rich. ex DC.) Hook. F. Ph. D. Universidade Estadual de Campinas, Campinas.

Goldenberg, R., F. Almeda, K. Sosa, R. C. Ribeiro, and F. A. Michelangeli. 2015. Rupestrea: A new brazilian genus of melastomataceae, with anomalous seeds and dry indehiscent fruits. Systematic Botany 40:561-571.

Goldenberg, R., and A. M. Amorim. 2006. Physeterostemon (Melastomataceae): a new genus and two new species from the Bahian Atlantic Forest, Brazil. Taxon 55:965-972.

Goldenberg, R., C. N. d. Fraga, A. P. Fontana, A. N. Nicolas, and F. A. Michelangeli. 2012. Taxonomy and phylogeny of Merianthera (Melastomataceae). Taxon 61:1040-1056. 
Goldenberg, R., D. S. Penneys, F. Almeda, W. S. Judd, and F. A. Michelangeli. 2008. Phylogeny of Miconia (Melastomataceae): Patterns of stamen diversification in a megadiverse neotropical genus. International Journal of Plant Sciences 169:963-979.

Goldenberg, R., M. Reginato, and F. A. Michelangeli. 2018. Disentangling the infrageneric classification of megadiverse taxa from Mata Atlantica: Phylogeny of Miconia section Chaenanthera (Melastomataceae: Miconieae). Taxon 67:537-551.

Goldenberg, R., and R. D. M. Tavares. 2007. A new species of Dolichoura (Melastomataceae) and broadened circumscription of the genus. Brittonia 59:226-232.

Grimm, D., and F. Almeda. 2013. Systematics, phylogeny, and biogeography of Chaetolepis (Melastomataceae). Journal of the Botanical Research Institute of Texas 7:217-263.

Guimarães, P. J. F., F. A. Michelangeli, K. Sosa, and J. R. de Santiago Gomez. 2019. Systematics of Tibouchina and allies (Melastomataceae: Melastomateae): A new taxonomic classification. Taxon 68:937-1002.

Howard, R. A. 1989. Flora of the Lesser Antilles; Dicotyledons (Part II).Vol. 5. Arnold Arboretum, Harvard University, Jamaica Plains, MA.

Ionta, G., W. Judd, J. Skean, and C. McMullen. 2012. Two new species of Miconia sect. Sagraea (Melastomataceae) from the Macaya Biosphere Reserve, Haiti, and twelve relevant new species combinations. Brittonia 64:61-72.

Ionta, G. M., W. S. Judd, N. H. Williams, and W. M. Whitten. 2007. Phylogenetic relationships in Rhexia (Melastomataceae): Evidence from DNA sequence data and morphology. International Journal of Plant Sciences 168:1055-1066.

Jacques-Félix, H. 1994. Histoire des Melastomataceae d'Afrique. Bulletin du Muséum National d'Histoire Naturelle Section B, Adansonia, 4e sér. 16:235-311.

Jørgensen, P. M., and S. León-Yánez. 1999. Catalogue of the vascular plants of Ecuador. Monographs in Systematic Botany from the Missouri Botanical Garden 75:1-1181.

Judd, W. S. 2007. Revision of Miconia sect. Chaenopleura (Miconieae, Melastomataceae) in the Greater Antilles. Systematic Botany Monographs 81:1-235.

Judd, W. S., E. R. Bécquer, J. J. D. Skean, and L. C. Majure. 2014a. Taxonomic studies in the Miconieae (Melastomataceae). XII. Revision of Miconia sect. Miconiastrum, with emphasis on the Miconia bicolor complex. Journal of the Botanical Research Institute of Texas 8:457-491.

Judd, W. S., E. R. Bécquer, and L. C. Majure. 2014b. Taxonomic studies in the Miconieae (Melastomataceae). XI. A revision of Miconia sect. Calycopteris on Hispaniola. Brittonia 66:216-249.

Judd, W. S., and G. M. Ionta. 2013. Taxonomic studies in the Miconieae (Melastomataceae). X. Revision of the species of the Miconia crotonifolia complex. Brittonia 65:66-95.

Judd, W. S., G. M. Ionta, and L. C. Majure. 2018a. Taxonomic studies in the Miconieae (Melastomataceae). XIV.Species of Miconia section Sagraea that occur in the Greater Antilles and additionally in the Lesser Antilles and/or continental regions. Journal of the Botanical Research Institute of Texas 12:531-547.

Judd, W. S., G. M. Ionta, L. C. Majure, and F. A. Michelangeli. 2018b. Taxonomic and Nomenclatural Notes on Miconia crenata and related species (Melastomataceae: Miconieae) in the Greater Antilles. Journal of the Botanical Research Institute of Texas 12:521-528.

Kartonegoro, A., P. Hovenkamp, and P. van Welzen. 2019. A taxonomic revision of Macrolenes (Melastomataceae). Garden's Bulletin Singapore 71:185-241. 
Kartonegoro, A., and J. F. Veldkamp. 2013. Revision of Creochiton (Melastomataceae). Blumea 58:217-227.

Kartonegoro, A., J. F. Veldkamp, P. Hovenkamp, and P. van Welzen. 2018. A revision of Dissochaeta (Melastomataceae, Dissochaeteae). PhytoKeys:1-178.

Kartonegoro, A., M. C. Veranso-Libalah, G. Kadereit, A. Frenger, D. S. Penneys, S. M. d. Oliveira, and P. C. V. Welzen. submitted. Molecular phylogenetics of the Dissochaetaalliance (Melastomataceae): Redefining tribe Dissochaeteae. . Taxon.

Kokubugata, G., K. Nakamura, W. H. Kuo, Z. C. Qi, K. F. Chung, C. X. Fu, Y. Suzuki, and M. Yokota. 2019. Reappraisal of Tashiroea as a genus inderpendent of Bredia (Melastomataceae) based on molecular data. Phytotaxa 392:75-83.

Koschnitzke, C., and A. B. Martins. 2006. Revisao taxonomica de Chaetostoma DC. (Melastomataceae, Microlicieae). Arquivos do Museu Nacional, Rio de Janeiro 64:95119.

Kral, R., and P. E. Bostick. 1969. The genus Rhexia (Melastomataceae). Sida 3:387-440.

Kriebel, R. 2008. Systematics and biogeography of the Neotropical genus Acisanthera (Melastomataceae). San Francisco State University, San Francisco.

Kriebel, R. 2012. A synopsis of the genus Poteranthera (Melastomeae: Melastomataceae) with the description of a new, apparently pollinator deceiving species. Brittonia 64:6-14.

Kriebel, R. 2016. A Monograph of Conostegia (Melastomataceae, Miconieae). PhytoKeys 67:1326.

Kriebel, R., F. A. Michelangeli, and L. M. Kelly. 2015. Discovery of unusual anatomical and continuous characters in the evolutionary history of Conostegia (Miconieae: Melastomataceae). Molecular Phylogenetics and Evolution 82:289-313.

Leon, B. 2006. Melastomataceae Endemicas del Peru. Revista Peruana de Biologia 13:428-452.

Liogier, H. A. 2000. Melastomataceae. Flora de la Española. Jardín Botánico Nacional Dr. Rafael Ma. Moscoso \& Instituto Tecnológico de Santo Domingo, Santo Domingo.

Lozano, G., and N. Becerra-de-Lozano. 1999. Los géneros Allomaieta y Cyphostyla (Melastomataceae). Revista de la Academia Colombiana de Ciencias 23:5-18.

Macbride, J. F. 1941. Melastomataceae. In Flora of Peru. Field Museum Publications in Botany 13:249-523.

Majure, L. C., and W. S. Judd. 2013. Miconia phrynosomaderma (Melastomataceae: Miconieae), A New Species From The Massif Du Nord, Haiti, And Sixteen New Names And Combinations. Journal of the Botanical Research Institute of Texas 7:265-274.

Majure, L. C., W. S. Judd, and F. A. Michelangeli. 2015a. Taxonomic revision of the Greater Antillean Pseudolima clade of Miconia (Miconia sect. Krugiophytum: Miconieae: Melastomataceae). Brittonia 67:11-28.

Majure, L. C., K. M. Neubig, J. D. Skean, Jr., E. R. Becquer, and W. S. Judd. 2015b. Evolution of the Sandpaper Clade (Miconieae, Melastomataceae) (vol 176, 607, 2015). International Journal of Plant Sciences 176:901-901.

Martin, C. V., D. P. Little, R. Goldenberg, and F. Michelangeli. 2008. A phylogenetic evaluation of Leandra (Miconieae, Melastomataceae): a polyphyletic genus where the seeds tell the story, not the petals. Cladistics 24:315-327.

Martins, A. B. 1984. Revisāo Taxonōmica do gênero Cambessedesia DC. (Melastomataceae). M. Sc. Universidade Estadual de Campinas, Campinas.

Martins, A. B. 1989. Revisāo Taxonōmica do gênero Marcetia DC. (Melastomataceae). Ph. D. Universidade Estadual de Campinas, Campinas. 
Martins, A. B., and F. Almeda. 2017. A Monograph of the Brazilian endemic genus Lavoisiera (Melastomataceae: Microlicieae). Phytotaxa 315:1-194.

Martins, E. 1997. Revisāo Taxonōmica do gênero Trembleya DC. (Melastomataceae). M. Sc. Universidade Estadual de Campinas, Campinas.

Maxwell, J. F. 1981. A revision of the genus Pternandra (Melastomataceae). Garden Bulletin (Singapore) 34:1-90.

Meirelles, J. 2015. Filogenia de Miconia seção Miconia subseção Seriatiflorae e revisão taxonômica do clado Albicans (Melastomataceae, Miconieae)

. Ph. D. UNICAMP, Campinas.

Mendoza-Cifuentes, H. 2020. Taxonomic revision of the genus Wurdastom (Melastomataceae: Cyphostyleae). Acta Botanica Mexicana 127:e1642.

Mendoza-Cifuentes, H., and J. L. Fernandez-Alonso. 2010. Evaluación de caracteres del cáliz y de los estambres en la tribu Merianieae (Melastomataceae) y definición de homologías. Revista de la Academia Colombiana de Ciencias Exactas 34:143-171.

Mendoza-Cifuentes, H., and J. L. Fernandez-Alonso. 2012. Novedades en Centronia y Meriania (Merianieae, Melastomataceae) y revisión taxonómica de Meriania grupo brachycera. Anales del Jardín Botánico de Madrid 69:259-294.

Michelangeli, F. A. 2005. A Monograph of Tococa Aublet (Melastomataceae). Flora Neotropica Monographs 98:1-114.

Michelangeli, F. A. 2014. Taxonomic notes on myrmecophilous Melastomataceae: A new species, two new synonyms, and an old species reconsidered. Brittonia 66:82-88.

Michelangeli, F. A., F. Almeda, M. Alvear, E. R. Becquer, J. Burke, M. K. Caddah, R. Goldenberg, G. M. Ionta, W. S. Judd, L. C. Majure, J. Meirelles, A. N. Nicolas, G. Ocampo, D. S. Penneys, J. D. Skean, and C. Ulloa Ulloa. 2016. (2462) Proposal to conserve Miconia, nom. cons. against the additional names Maieta and Tococa (Melastomataceae: Miconieae). Taxon 65:892-893.

Michelangeli, F. A., and R. Goldenberg. 2016. Miconia papillosperma (Melastomataceae, Miconieae): a new species from Amazonas, Brazil. PhytoKeys 63:31-40.

Michelangeli, F. A., R. Goldenberg, F. Almeda, W. S. Judd, E. R. Bécquer, G. Ocampo, G. M. Ionta, J. D. S. Jr., L. C. Majure, and D. S. Penneys. 2019. Nomenclatural novelties in Miconia (Melastomataceae: Miconieae). Brittonia:81-121 [published online 110/115/2018].

Michelangeli, F. A., W. S. Judd, D. S. Penneys, J. D. Skean, E. R. Bécquer-Granados, R. Goldenberg, and C. V. Martin. 2008. Multiple events of dispersal and radiation of the tribe Miconieae (Melastomataceae) in the Caribbean. Botanical Review 74:53-77.

Michelangeli, F. A., and W. Meier. 2013. A new anisophyllous species of Miconia (Melastomataceae: Miconieae) from the Coastal Cordillera in northern Venezuela. Phytotaxa 79:37-44.

Michelangeli, F. A., A. Nicolas, M. E. Morales-P, and H. David. 2011. Phylogenetic Relationships of Allomaieta, Alloneuron, Cyphostyla, and Wurdastom (Melastomataceae) and the Resurrection of the Tribe Cyphostyleae. International Journal of Plant Sciences 172:1165.

Michelangeli, F. A., P. J. F. Guimaraes, D. S. Penneys, F. Almeda \& R. Kriebel. 2013. Phylogenetic relationships and distribution of New World Melastomeae (Melastomataceae). Botanical Journal of the Linnean Society 171:38-60. 
Michelangeli, F. A., D. S. Penneys, J. Giza, D. Soltis, M. H. Hils, and J. D. Skean, Jr. 2004. A preliminary phylogeny of the tribe Miconieae (Melastomataceae) based on nrITS sequence data and its implications on inflorescence position. Taxon 53:279-290.

Michelangeli, F. A., and C. Ulloa Ulloa. 2016. A new species of Alloneuron (Melastomataceae) from northern Peru. Brittonia 68:429-432.

Michelangeli, F. A., C. Ulloa Ulloa, and K. Sosa. 2014. Quipuanthus, a new genus of Melastomataceae from the foothills of the Andes in Ecuador and Peru. Systematic Botany 39:533-540.

Morales, M. E. 2010. Análisis filogenético de Huilaea Wurdack (Melastomataceae) basado en datos morfológicos y moleculares. Ph. D. Universidad Nacional de Colombia, Bogota.

Morales, M. E., and F. González. 2005. Redescubrimiento de Chalybea Naudin e implicaciones en la delimitación genérica de Huilaea Wurdack (Melastomataceae). Revista de la Academia Colombiana de Ciencias Exactas 29:171-178.

Morley, T. 1976. Memecylae (Melastomataceae). Flora Neotropica Monographs 15:1-295.

Penneys, D. S. 2001. A systematic revision and cladistic analysis of Charianthus (Miconieae : Melastomataceae) using morphological and molecular characters. M. Sc. University of Florida, Gainesville, FL.

Penneys, D. S. 2007. Phylogeny and character evolution in the Blakeeae (Melastomataceae): Neotropical hemiepiphytes with mite

and ant domatia. . Ph. D. University of Florida, Gainesville, FL.

Penneys, D. S. 2013. Preliminary phylogeny of the Astronieae (Melastomataceae) Based on Nuclear and Plastid DNA Sequence Data, with Comments on the Philippine Endemic Genus, Astrocalyx. Phillipine Journal of Science 142:159-168.

Penneys, D. S., F. Almeda, F. A. Michelangeli, R. Goldenberg, A. B. Martins, and P. W. Fritsch. 2020. Lithobieae and Eriocnemeae: two new neotropical tribes of Melastomataceae. Phytotaxa 453:157-178.

Penneys, D. S., and W. S. Judd. 2013a. Combined Molecular and Morphological Phylogenetic Analyses of the Blakeeae (Melastomataceae). International Journal of Plant Sciences 174:802-817.

Penneys, D. S., and W. S. Judd. 2013b. A revised circumscription for the Blakeeae (Melastomataceae) with associated nomenclatural adjustments. PhytoKeys 20:17-32.

Penneys, D. S., F. A. Michelangeli, W. S. Judd, and F. Almeda. 2010. Henriettieeae: A new Neotropical tribe of berry-fruited Melastomataceae. Systematic Botany 35:783-800.

Reginato, M. 2016. Taxonomic revision of Leandra sect. Leandra (Melastomataceae, Miconieae). Phytotaxa 262:1-97.

Reginato, M., and F. A. Michelangeli. 2016. Untangling the phylogeny of Leandra s.str. (Melastomataceae, Miconieae). Molecular Phylogenetics and Evolution 96:17-32.

Reginato, M., K. M. Neubig, L. C. Majure, and F. A. Michelangeli. 2016. The first complete plastid genomes of Melastomataceae are highly structurally conserved. PeerJ 4.

Renner, S. S. 1987. Sandemania Hoehnei (Melastomataceae: Tibouchineae): Taxonomy, Distribution, and Biology. Brittonia 39:441-446.

Renner, S. S. 1989. Systematic studies in the Melastomataceae Bellucia, Loreya and Macairea. Memoirs of The New York Botanical Garden 50:1-112.

Renner, S. S. 1990. A Revision of Rhynchanthera Melastomataceae. Nordic Journal of Botany 9:601-630. 
Renner, S. S. 1993. Phylogeny and classification of the Melastomataceae and Memecylaceae. Nordic Journal of Botany 13:519-540.

Renner, S. S. 1994a. A revision of Pterolepis (Melastomataceae: Melastomeae). Nordic Journal of Botany 14:73-104.

Renner, S. S. 1994b. Revisions of Pterogastra and Schwackaea (Melastomataceae: Melastomeae). Nordic Journal of Botany 14:65-71.

Renner, S. S. 2004a. Bayesian analysis of combined chloroplast loci, using multiple calibrations, supports the recent arrival of Melastomataceae in Africa and Madagascar. American Journal of Botany 91:1427-1435.

Renner, S. S. 2004b. Multiple Miocene Melastomataceae dispersal between Madagascar, Africa and India. Philosophical Transactions of The Royal Society of London Series BBiological Sciences 359:1485-1494.

Renner, S. S., G. Clausing, and K. Meyer. 2001. Historical biogeography of Melastomataceae: the roles of Tertiary migration and long-distance dispersal. American Journal of Botany 88:1290-1300.

Renner, S. S., and K. Meyer. 2001. Melastomeae come full circle: Biogeographic reconstruction and molecular clock dating. Evolution 55:1315-1324.

Rocha, M. J. R., J. A. N. Batista, P. J. F. Guimaraes, and F. A. Michelangeli. 2016 a. Phylogenetic relationships in the Marcetia alliance (Melastomeae, Melastomataceae) and implications for generic circumscription. Botanical Journal of the Linnean Society 181:585-609.

Rocha, M. J. R., P. J. Guimarães, F. A. Michelangeli, and J. A. Nogueira Batista. 2018. Taxonomy of Marcetieae: A New Neotropical Tribe of Melastomataceae. International Journal of Plant Sciences 179:50-74.

Rocha, M. J. R., P. J. F. Guimaraes, F. A. Michelangeli, and R. Romero. 2016b. Phylogenetic placement and a new circumscription of Poteranthera (Microlicieae; Melastomataceae). Phytotaxa 263:219-232.

Romero, R., and A. F. Alves Versiane. 2014. Taxonomic Novelty and Typifications in Microlepis (Melastomataceae). Novon 23:217-223.

Schulman, L., and J. Hyvonen. 2003. A cladistic analysis of Adelobotrys (Melastomataceae) based on morphology, with notes on generic limits within the tribe Merianieae. Systematic Botany 28:738-756.

Seco, R. S. 2006. Estudos taxonômicos no gênero Comolia DC. (MelastomataceaeMelastomeae) no Brasil. . M. Sc. Universidade Estadual de Campinas, Campinas.

Skean, J. D., Jr. 1993. Monograph of Mecranium (Melastomatacae-Miconiae). Systematic Botany Monographs 39:1-116.

Skean, J. D., W. S. Judd, L. C. Majure, and G. M. Ionta. 2018. Recognition of Miconia sect. Sagraeoides (Melastomataceae: Miconieae) and associated nomenclatural changes. Brittonia 70:342-355.

Stone, R. D. 2005. Phylogeny of Major Lineages in Melastomataceae, Subfamily Olisbeoideae: Utility of Nuclear Glyceraldehyde 3-Phosphate Dehydrogenase (GapC) Gene Sequences. Systematic Botany 31:107-121.

Tavares, R. D. M. 2005. Revisāo Taxonōmica do gênero Behuria Cham. (Melastomataceae, Merianieae). M. Sc. Universidade Federal do Rio de Janeiro, Rio de Janeiro. 
Todzia, C. A., and F. Almeda. 1991. A revision of Tibouchina section Lepidotae (Melastomataceae: Tibouchineae). Proceedings of the California Academy of Sciences 47:175-206.

Triana, J. 1871. Les Melastomacées. Transactions of the Linnean Society of London 28:1-188.

Triana, J. J. 1866. Dispositio Melastomacearum. Bulletin du Congres International de Botanique et d'Horticulture reuni a Amsterdam 1865:457-461.

Ulloa Ulloa, C., P. Acevedo-Rodriguez, S. Beck, M. J. Belgrano, R. Bernal, P. E. Berry, L. Brako, M. Celis, G. Davidse, R. C. Forzza, S. R. Gradstein, O. Hokche, B. Leon, S. Leon-Yanez, R. E. Magill, D. A. Neill, M. Nee, P. H. Raven, H. Stimmel, M. T. Strong, J. L. Villasenor, J. L. Zarucchi, F. O. Zuloaga, and P. M. Jorgensen. 2017. An integrated assessment of the vascular plant species of the Americas. Science 358:1614-1617.

Veranso-Libalah, M. C., G. Kadereit, R. D. Stone, and T. L. P. Couvreur. 2018. Multiple shifts to open habitats in Melastomateae (Melastomataceae) congruent with the increase of African Neogene climatic aridity. Journal of Biogeography 45:1420-1431.

Veranso-Libalah, M. C., O. Lachenaud, R. D. Stone, and G. Kadereit. 2019. Nothodissotis (Melastomataceae), a new genus from Atlantic Central Africa, including the new species N-alenensis from Equatorial Guinea. PhytoKeys:89-103.

Veranso-Libalah, M. C., R. D. Stone, A. G. N. Fongod, T. L. P. Couvreur, and G. Kadereit. 2017. Phylogeny and systematics of African Melastomateae (Melastomataceae). Taxon 66:584-614.

Veranso-Libalah, M. C., R. D. Stone, and G. Kadereit. 2020. Towards a complete phylogeny of African Melastomateae: Systematics of Dissotis and allies (Melastomataceae). Taxon 69.

Wallnöfer, B. 1996. A revision of the genus Alloneuron Pilg. and segregation of Wurdastom gen.n. (Melastomataceae). Annalen des Naturhistorischen Museums in Wien Serie B Botanik und Zoologie 98:447-462.

Wallnöfer, B. 1999. Alloneuron Pilg. (Melastomataceae): some additions. Annalen des Naturhistorischen Museums in Wien Serie B Botanik und Zoologie 101B:593-598.

Warner, R. H. 2002. Systematics of the genus Monolena (Melastomataceae) in Central America. Proceedings of the California Academy of Sciences 53:95-116.

Whiffin, T. P. 1972. A systematic study of the genus Heterocentron (Melastomataceae). Ph. D. University of Texas, Austin.

Wurdack, J. J. 1953. A revision of the genus Brachyotum (Tibouchineae-Melastomataceae). Memoirs of The New York Botanical Garden 8:343-407.

Wurdack, J. J. 1973. Melastomataceae. Pages 1-819 in T. Lasser, editor. Flora de Venezuela. Instituto Botánico, Caracas.

Wurdack, J. J. 1977. Certamen Melastomataceis XXVI. Phytologia 35:241-251.

Wurdack, J. J. 1980. Melastomataceae. Page 406 in G. Harling and B. Sparre, editors. Flora of Ecuador No. 13. University of Goteborg, Swedish Natural Science Research Council, Stockohlm.

Wurdack, J. J., S. S. Renner, and T. Morley. 1993. Melastomataceae. Flora of the Guianas. Koeltz Scientific Books, Koenigstein, Germany.

Wurdack, K. J., and F. A. Michelangeli. 2019. Systematics and relationships of Tryssophyton (Melastomataceae), with a second species from the Pakaraima Mountains of Guyana. PhytoKeys:1-21.

Zhou, Q., C.-W. Lin, W. L. Ng, J. Dai, T. Denda, R. Zhou, and Y. Liu. 2019a. Analyses of Plastome Sequences Improve Phylogenetic Resolution and Provide New Insight Into the 
Evolutionary History of Asian Sonerileae/Dissochaeteae. Frontiers in Plant Science 10:1477.

Zhou, Q. J., J. H. Dai, C. W. Lin, T. Denda, R. C. Zhou, and Y. Liu. 2019b. Recircumscription of Bredia and resurrection of Tashiroea (Sonerileae, Melastomataceae) with description of a new species T. villosa. PhytoKeys:121-150. 
Table 1: Linear sequence of Melastomataceae genera, with their tribal assignment and estimated number of species.

\begin{tabular}{|c|c|c|c|c|c|}
\hline Tribe/Clade & $\begin{array}{l}\text { Linear } \\
\text { Sequence }\end{array}$ & Genus & Authority & Distribution & \# spp \\
\hline Olisbeoideae & 1.10 & Lijndenia & Zoll. \& Moritzi & Old World & 16 \\
\hline Olisbeoideae & 1.20 & Memecylon & L. & Old World & 379 \\
\hline Olisbeoideae & 1.30 & Spathandra & Guill. \& Perr. & Old World & \\
\hline Olisbeoideae & 1.40 & Warneckea & Gilg & Old World & 49 \\
\hline Olisbeoideae & 1.50 & Mouriri & Aubl. & New World & \\
\hline Olisbeoideae & 1.60 & Votomita & Aubl. & New World & \\
\hline Kibessieae & 2.10 & Pternandra & Jack & Old World & \\
\hline Astronieae & 3.10 & Tessmannianthus & Markgr. & New World & \\
\hline Astronieae & 3.20 & Astrocalyx & Merr. & Old World & \\
\hline Astronieae & 3.30 & Astronia & Blume & Old World & \\
\hline Astronieae & 3.40 & Astronidium & A.Gray & Old World & \\
\hline Astronieae & 3.50 & Beccarianthus & Cogn. & Old World & 1 \\
\hline Lithobieae & 4.10 & Lithobium & Bong. & New World & \\
\hline Henrietteeae & 5.10 & Bellucia & Neck. ex Raf. & New World & \\
\hline Henrietteeae & 5.20 & Henriettea & DC. & New World & \\
\hline Henrietteeae & 5.30 & Kirkbridea & Wurdack & New World & ] \\
\hline Blakeeae & 6.10 & Chalybea & Naudin & New World & 11 \\
\hline Blakeeae & 6.20 & Blakea & Browne & New World & 19 \\
\hline Merianieae & 7.10 & Macrocentrum & Hook.f. & New World & \\
\hline Merianieae & 7.20 & Maguireanthus & Wurdack & New World & \\
\hline Merianieae & 7.30 & Salpinga & Mart. ex DC. & New World & \\
\hline Merianieae & 7.40 & Adelobotrys & DC. & New World & 3 \\
\hline Merianieae & 7.50 & Graffenrieda & DC. & New World & \\
\hline Merianieae & 7.60 & Meriania & Sw. & New World & 12 \\
\hline Merianieae & 7.70 & Axinaea & Ruiz \& Pav. & New World & \\
\hline Merianieae & 7.80 & Centronia & D.Don & New World & \\
\hline Eriocnemeae & 8.10 & Ochthephilus & Wurdack & New World & \\
\hline Eriocnemeae & 8.20 & Eriocnema & $\begin{array}{l}\text { Naudin } \\
\text { R.Goldenb. \& }\end{array}$ & New World & \\
\hline Eriocnemeae & 8.30 & Physeterostemon & Amorim & New World & \\
\hline Miconieae & 9.10 & Miconia & Ruiz \& Pav. & New World & 1901 \\
\hline Unplaced & 10.10 & Dinophora & Benth. & Old World & \\
\hline Unplaced & 10.20 & Ochthocharis & Blume & Old World & \\
\hline Bertolonieae & 11.10 & Bertolonia & Raddi & New World & \\
\hline Sonerileae & 12.10 & Boyania & Wurdack & New World & \\
\hline Sonerileae & 12.20 & Opisthocentra & Hook.f. & New World & \\
\hline Sonerileae & 12.30 & Phainantha & Gleason & New World & \\
\hline Sonerileae & 12.40 & Tryssophyton & Wurdack & New World & \\
\hline
\end{tabular}




\begin{tabular}{|c|c|c|c|c|}
\hline Sonerileae & 12.50 & Neblinanthera & Wurdack & New World \\
\hline Sonerileae & 12.60 & Tateanthus & Gleason & New World \\
\hline Sonerileae & 12.70 & Amphiblemma & Naudin & Old World \\
\hline Sonerileae & 12.80 & Calvoa & J. D. Hooker & Old World \\
\hline Sonerileae & 12.90 & Cincinnobotrys & Gilg & Old World \\
\hline Sonerileae & 12.100 & Dicellandra & J. D. Hooker & Old World \\
\hline Sonerileae & 12.110 & Gravesia & Naudin & Old World \\
\hline Sonerileae & 12.120 & Preussiella & $\begin{array}{l}\text { Gilg. } \\
\text { Gaudichaud- }\end{array}$ & Old World \\
\hline Sonerileae & 12.130 & Medinilla & Beupre & Old World \\
\hline Sonerileae & 12.140 & Allomorphia & Blume & Old World \\
\hline Sonerileae & 12.150 & Anerincleistus & P. W. Korthals & Old World \\
\hline Sonerileae & 12.160 & Aschistanthera & C. Hansen & Old World \\
\hline Sonerileae & 12.170 & Barthea & J. D. Hooker & Old World \\
\hline Sonerileae & 12.180 & Blastus & Loureiro & Old World \\
\hline Sonerileae & 12.190 & Boerlagea & Cogn. & Old World \\
\hline Sonerileae & 12.200 & Bredia & Blume & Old World \\
\hline Sonerileae & 12.210 & Brittenia & Cogn. & Old World \\
\hline Sonerileae & 12.220 & Campimia & C. Hansen & Old World \\
\hline Sonerileae & 12.230 & Catanthera & F.V. Muller & Old World \\
\hline Sonerileae & 12.240 & Cyanandrium & Stapf & Old World \\
\hline Sonerileae & 12.250 & Cyphotheca & Diels & old World \\
\hline Sonerileae & 12.260 & Driessenia & P. W. Korthals & Old World \\
\hline Sonerileae & 12.270 & Enaulophyton & Steenis & Old World \\
\hline Sonerileae & 12.280 & Fordiophyton & $\begin{array}{l}\text { Bullock } \\
\text { (Blume) Cámara- } \\
\text { Leret, Ridd.-Num. }\end{array}$ & Old World \\
\hline Sonerileae & 12.290 & Heteroblemma & \& Veldk. & Old World \\
\hline Sonerileae & 12.300 & Kendrickia & J. D. Hooker & Old World \\
\hline Sonerileae & 12.310 & Kerriothyrsus & C. Hansen & Old World \\
\hline Sonerileae & 12.320 & Neodriessenia & Nayar & Old World \\
\hline Sonerileae & 12.330 & Oxyspora & DC. & Old World \\
\hline Sonerileae & 12.340 & Pachycentria & Blume & Old World \\
\hline Sonerileae & 12.350 & Phyllagathis & Blume & Old World \\
\hline Sonerileae & 12.360 & Plagiopetalum & Rehd. & Old World \\
\hline Sonerileae & 12.370 & Plethiandra & J. D. Hooker & Old World \\
\hline Sonerileae & 12.380 & Pogonanthera & Blume & Old World \\
\hline Sonerileae & 12.400 & Poikilogyne & E.G.Baker & Old World \\
\hline Sonerileae & 12.410 & Poilannammia & C. Hansen & Old World \\
\hline Sonerileae & 12.420 & Sarcopyramis & Wallich & Old World \\
\hline Sonerileae & 12.430 & Scorpiothyrsus & Hui-Lin Li & Old World \\
\hline
\end{tabular}




\begin{tabular}{|c|c|c|c|c|}
\hline Sonerileae & 12.440 & Sonerila & Roxburgh & Old World \\
\hline Sonerileae & 12.450 & Sporoxeia & W.W.Smith & Old World \\
\hline Sonerileae & 12.460 & Stussenia & C. Hansen & Old World \\
\hline Sonerileae & 12.470 & Styrophyton & $\mathrm{Hu}$ & old World \\
\hline Sonerileae & 12.480 & Tashiroea & Matsum. & Old World \\
\hline Sonerileae & 12.490 & Trigidiopalma & Chen & Old World \\
\hline Sonerileae & 12.500 & Tylanthera & C. Hansen & Old World \\
\hline Sonerileae & 12.510 & Vietsenia & C. Hansen & Old World \\
\hline Unplaced & 13.10 & Feliciadamia & $\begin{array}{l}\text { Bullock } \\
\text { Triana ex Benth. }\end{array}$ & Old World \\
\hline Trioleneae & 14.10 & Monolena & \& Hook.f. & New World \\
\hline Trioleneae & 14.20 & Triolena & Naudin & New World \\
\hline Cyphostyleae & 15.10 & Wurdastom & B.Walln. & New World \\
\hline Cyphostyleae & 15.20 & Allomaieta & Gleason & New World \\
\hline Cyphostyleae & 15.30 & Alloneuron & $\begin{array}{l}\text { Pilg. } \\
\text { Michelang. \& }\end{array}$ & New World \\
\hline Cyphostyleae & 15.40 & Quipuanthus & C.Ulloa & New World \\
\hline Cambessedesieae & 16.10 & Cambessedesia & DC. & New World \\
\hline Cambessedesieae & 16.20 & Merianthera & Kuhlm. & New World \\
\hline Cambessedesieae & 16.30 & Huberia & DC. & New World \\
\hline Cambessedesieae & 16.40 & Bisglaziovia & Cogn. & New World \\
\hline Dissochaeteae & 17.30 & Dalenia & Korth. & Old World \\
\hline Dissochaeteae & 17.60 & Dissochaeta & $\begin{array}{l}\text { Blume } \\
\text { (Blume) }\end{array}$ & Old World \\
\hline Dissochaeteae & 17.40 & Diplectria & H.G.L.Reichnbach & Old World \\
\hline Dissochaeteae & 17.50 & Macrolenes & Naudin ex Miquel & Old World \\
\hline Dissochaeteae & 17.20 & Creochiton & Blume & Old World \\
\hline Dissochaeteae & 17.10 & Pseudodissochaeta & $\begin{array}{l}\text { Nayar } \\
\text { R.Goldenb., } \\
\text { Almeda \& }\end{array}$ & Old World \\
\hline Unplaced & 18.10 & Rupestrea & Michelang. & New World \\
\hline Rhexieae & 19.10 & Pachyloma & DC. & New World \\
\hline Rhexieae & 19.20 & Arthrostemma & Pav. ex D. Don & New World \\
\hline Rhexieae & 19.30 & Rhexia & L. & New World \\
\hline Microliceae & 20.10 & Rhynchanthera & DC. & New World \\
\hline Microliceae & 20.20 & Poteranthera & Bong. & New World \\
\hline Microliceae & 20.30 & Microlicia & D. Don & New World \\
\hline Microliceae & 20.40 & Lavoisiera & DC. & New World \\
\hline Microliceae & 20.50 & Chaetostoma & DC. & New World \\
\hline Microliceae & 20.60 & Trembleya & DC. & New World \\
\hline Microliceae & 20.70 & Stenodon & Naudin & New World \\
\hline Marcetieae & 21.10 & Comoliopsis & Wurdack & New World \\
\hline
\end{tabular}




\begin{tabular}{|c|c|c|c|c|}
\hline Marcetieae & 21.20 & Pseudoernestia & Krasser & New World \\
\hline Marcetieae & 21.30 & Dicrananthera & C.Presl & New World \\
\hline Marcetieae & 21.40 & Comolia & DC. & New World \\
\hline Marcetieae & 21.50 & Mallophyton & Wurdack & New World \\
\hline Marcetieae & 21.60 & Acanthella & $\begin{array}{l}\text { Hook.f. } \\
\text { M.J.Rocha \& }\end{array}$ & New World \\
\hline Marcetieae & 21.70 & Rostranthera & P.J.F.Guim. & New World \\
\hline Marcetieae & 21.80 & Noteropila & Mart. & New World \\
\hline Marcetieae & 21.90 & Acisanthera & P.Browne & New World \\
\hline Marcetieae & 21.100 & Sandemania & Gleason & New World \\
\hline Marcetieae & 21.110 & Leiostegia & Benth. & New World \\
\hline Marcetieae & 21.120 & Siphanthera & Pohl & New World \\
\hline Marcetieae & 21.130 & Macairea & DC. & New World \\
\hline Marcetieae & 21.140 & Ernestia & DC. & New World \\
\hline Marcetieae & 21.150 & Appendicularia & $\begin{array}{l}\text { DC. } \\
\text { Almeda \& }\end{array}$ & New World \\
\hline Marcetieae & 21.160 & Brasilianthus & Michelang. & New World \\
\hline Marcetieae & 21.170 & Nepsera & Naudin & New World \\
\hline Marcetieae & 21.180 & Fritzschia & Cham. & New World \\
\hline Marcetieae & 21.190 & Aciotis & D. Don & New World \\
\hline Marcetieae & 21.200 & Marcetia & DC. & New World \\
\hline Melastomateae & 22.10 & Loricalepis & Brade & New World \\
\hline Melastomateae & 22.20 & Pterolepis & (DC.) Miq. & New World \\
\hline Melastomateae & 22.30 & Pterogastra & Naudin & New World \\
\hline Melastomateae & 22.40 & Desmoscelis & Naudin & New World \\
\hline Melastomateae & 22.50 & Centradenia & $\begin{array}{l}\text { G. Don } \\
\text { Almeda \& }\end{array}$ & New World \\
\hline Melastomateae & 22.60 & Pilocosta & Whiffin & New World \\
\hline Melastomateae & 22.70 & Heterocentron & Hook. \& Arn. & New World \\
\hline Melastomateae & 22.80 & Schwackaea & Cogn. & New World \\
\hline Melastomateae & 22.90 & Bucquetia & DC. & New World \\
\hline Melastomateae & 22.100 & Castratella & Naudin & New World \\
\hline Melastomateae & 22.110 & Chaetolepis & (DC.) Miq. & New World \\
\hline Melastomateae & 22.120 & Monochaetum & $\begin{array}{l}\text { (DC.) Naudin } \\
\text { P.J.F.Guim. \& }\end{array}$ & New World \\
\hline Melastomateae & 22.130 & Andesanthus & Michelang. & New World \\
\hline Melastomateae & 22.140 & Tibouchina & Aubl. & New World \\
\hline Melastomateae & 22.150 & Pleroma & D. Don & New World \\
\hline Melastomateae & 22.160 & Chaetogastra & D. Don & New World \\
\hline Melastomateae & 22.170 & Brachyotum & (DC.) Triana & New World \\
\hline Melastomateae & 22.180 & Guyonia & $\begin{array}{l}\text { Naudin } \\
\text { Ver.-Lib. \& }\end{array}$ & Old World \\
\hline Melastomateae & 22.190 & Anaheterotis & G.Kadereit & Old World \\
\hline
\end{tabular}




\begin{tabular}{|c|c|c|c|c|}
\hline Melastomateae & 22.200 & Argyrella & Naudin & Old World \\
\hline Melastomateae & 22.210 & Tristemma & Juss & Old World \\
\hline Melastomateae & 22.220 & Melastomastrum & Naudin & Old World \\
\hline Melastomateae & 22.230 & Cailliella & Jacq-Fél. & Old World \\
\hline Melastomateae & 22.240 & Dichaetanthera & $\begin{array}{l}\text { Endl. } \\
\text { (A.Fern. \& } \\
\text { R.Fern.) Veranso- } \\
\text { Libalah \& }\end{array}$ & Old World \\
\hline Melastomateae & 22.250 & Dissotidendron & G.Kadereit & Old World \\
\hline Melastomateae & 22.260 & Heterotis & Benth. & Old World \\
\hline Melastomateae & 22.270 & Dupineta & $\begin{array}{l}\text { (Sm.) Raf. } \\
\text { Veranso-Libalah }\end{array}$ & Old World \\
\hline Melastomateae & 22.280 & Nothodissotis & \& G.Kadereit & Old World \\
\hline Melastomateae & 22.290 & Pseudosbeckia & $\begin{array}{l}\text { A.Fern. \& R.Fern. } \\
\text { Ver.-Lib. \& }\end{array}$ & Old World \\
\hline Melastomateae & 22.300 & Rosettea & G.Kadereit & Old World \\
\hline Melastomateae & 22.310 & Derosiphia & Raf. & Old World \\
\hline Melastomateae & 22.320 & Dissotis & Benth. & Old World \\
\hline Melastomateae & 22.330 & Nerophila & $\begin{array}{l}\text { Naudin } \\
\text { (Naudin) J. D. }\end{array}$ & Old World \\
\hline Melastomateae & 22.340 & Antherotoma & $\begin{array}{l}\text { Hooker } \\
\text { Ver.-Lib. \& }\end{array}$ & Old World \\
\hline Melastomateae & 22.350 & Almedanthus & $\begin{array}{l}\text { R.D.Stone } \\
\text { Ver.-Lib. \& }\end{array}$ & Old World \\
\hline Melastomateae & 22.360 & Eleotis & $\begin{array}{l}\text { R.D.Stone } \\
\text { Ver.-Lib. \& }\end{array}$ & Old World \\
\hline Melastomateae & 22.370 & Pyrotis & $\begin{array}{l}\text { R.D.Stone } \\
\text { Ver.-Lib. \& }\end{array}$ & Old World \\
\hline Melastomateae & 22.380 & Feliciotis & G.Kadereit & Old World \\
\hline Melastomateae & 22.390 & Dionychastrum & A.Fern. \& R.Fern. & Old World \\
\hline Melastomateae & 22.400 & Amphorocalyx & J. G. Baker & Old World \\
\hline Melastomateae & 22.410 & Dionycha & Naudin & Old World \\
\hline Melastomateae & 22.420 & Rousseauxia & DC. & Old World \\
\hline Melastomateae & 22.430 & Melastoma & L. & Old World \\
\hline Melastomateae & 22.440 & Osbeckia & L. & Old World \\
\hline Melastomateae & 22.450 & Otanthera & Blume & Old World \\
\hline Unplaced & 23.10 & Centradeniastrum & Cogn. & New World \\
\hline Unplaced & 23.20 & Stanmarkia & Almeda & New World \\
\hline
\end{tabular}


Table 2: New genera of New World Melastomataceae published since 2005.

$\begin{array}{llll}\text { Genus } & \text { Status } & \text { Tribe } & \begin{array}{l}\text { Species } \\ \text { former } \\ \text { placement }\end{array} \\ \text { Andesanthus } & \text { New } & \text { Melastomatoideae } & \text { Tibouchina } \\ \text { Brasilianthus } & \text { New } & \text { Marcetieae } & \text { NEW } \\ \text { Chaetogastra } & \text { Resurrected } & \text { Melastomatoideae } & \text { Tibouchina } \\ \text { Dicrananthera } & \text { Resurrected } & \text { Marcetieae } & \text { Acisanthera } \\ \text { Leiostegia } & \text { Resurrected } & \text { Marcetieae } & \text { Comolia } \\ \text { Noterophila } & \text { Resurrected } & \text { Marcetieae } & \text { Acisanthera } \\ \text { Physeterostemon } & \text { New } & \text { Eriocnemeae } & \text { NEW } \\ \text { Pleroma } & \text { Resurrected } & \text { Melastomatoideae } & \text { Tibouchina } \\ \text { Pseudoernestia } & \text { Resurrected } & \text { Marcetieae } & \text { Ernestia } \\ \text { Quipuanthus } & \text { New } & \text { Cyphostyleae } & \text { NEW } \\ \text { Rostranthera } & \text { New } & \text { Marcetieae } & \text { Acisanthera } \\ \text { Rupestrea } & \text { New } & \text { unplaced } & \text { Miconia }\end{array}$


Table 3: Genera of New World Melastomataceae synonymized since 2005

\begin{tabular}{|c|c|c|}
\hline Tribe & Genus & Currently Accepted as: \\
\hline Blakeeae & Huilaea & Chalybea \\
\hline Blakeeae & Topobea & Blakea \\
\hline Cambessedessiae & Behuria & Huberia \\
\hline Cambessedessiae & Benevidesia & Huberia \\
\hline Cambessedessiae & Dolichoura & Huberia \\
\hline Henrietteeae & Henriettella & Henriettea \\
\hline Henrietteeae & Llewellynia & Henriettea \\
\hline Henrietteeae & Loreya & Bellucia \\
\hline Henrietteeae & Myriaspora & Bellucia \\
\hline Melastomateae & Itatiaia & Pleroma \\
\hline Melastomateae & Microlepis & Pleroma \\
\hline Melastomateae & Schizocentron & Heterocentron \\
\hline Melastomateae & Svitramia & Pleroma \\
\hline Melastomateae & Tibouchinopsis & Pleroma \\
\hline Miconieae & Anaectocalyx & Miconia \\
\hline Miconieae & Calycogonium & Miconia \\
\hline Miconieae & Catocoryne & Miconia \\
\hline Miconieae & Charianthus & Miconia \\
\hline Miconieae & Clidemia & Miconia \\
\hline Miconieae & Conostegia & Miconia \\
\hline Miconieae & Heterotrichum & Miconia \\
\hline Miconieae & Icaria & Miconia \\
\hline Miconieae & Killipia & Miconia \\
\hline Miconieae & Leandra & Miconia \\
\hline Miconieae & Mecranium & Miconia \\
\hline Miconieae & Necranium & Miconia \\
\hline Miconieae & Ossaea & Miconia \\
\hline Miconieae & Pachyanthus & Miconia \\
\hline Miconieae & Pentossaea & Miconia \\
\hline Miconieae & Platycentrum & Miconia \\
\hline Miconieae & Pleiochiton & Miconia \\
\hline Miconieae & Sagraea & Miconia \\
\hline Miconieae & Tetrazygia & Miconia \\
\hline Miconieae & Tococa & Miconia \\
\hline Triolenae & Diolena & Triolena \\
\hline Triolenae & Diplarpea & Triolena \\
\hline
\end{tabular}


Table 4: Formerly and currently accepted names for species of New World Melastomataceae that have changed genera since 2010 .

\section{Formely accepted name}

Acisanthera bivalvis (Aubl.) Cogn.

Acisanthera crassipes (Naudin) Wurdack

Acisanthera genliseoides (Hoehne) Wurdack

Acisanthera hedyotoidea (C.Presl.) Triana

Acisanthera limnobios (DC.) Triana

Acisanthera nana Ule

Acisanthera quadrata Pers.

Acisanthera rosulans Huber

Acisanthera tetraptera (Cogn.) Gleason

Adelobotrys antioquiensis Wurdack

Anaectocalyx bracteosa (Naudin) Triana ex Cogn.

Anaectocalyx latifolia Cogn.

Anaectocalyx manarae Wurdack

Appendicularia rubra (Pulle.) M.J.R.Rocha \&

P.J.F.Guim.

Behuria capixaba R.Goldenb. \& Reginato

Behuria comosa R. Tavares, Baumgratz \&

R.Goldenb.

Behuria cordifolia Cogn.

Behuria corymbosa Cogn.

Behuria edmundoi Brade

Behuria glaziouviana Cogn.

Behuria glutinosa Cogn.

Behuria huberioides Brade

Behuria insignis Cham.

Behuria limae Brade

Behuria lumiarensis Bochorny, Michelang. \&

R.Goldenb.

Behuria magdalenensis (Brade) R. Tavares \&

Baumgratz

Behuria mestrealvarensis D.T. Iglesias \& R.Goldenb.

Behuria mouraei Cogn.

Behuria organensis (Saldanha \& Cogn.) R. Tavares

\& Baumgratz

Behuria parvifolia Cogn.

Behuria souza-limae Brade

\section{Currently accepted name}

Noterophila bivalvis (Aubl.) Kriebel \& M.J.R.Rocha

Noterophila crassipes (Naudin) Kriebel \& M.J.R.Rocha

Noterophila genliseoides (Hoehne) Kriebel \&

M.J.R.Rocha

Dicrananthera hedyotoidea C.Presl.

Noterophila limnobios (DC.) Mart.

Noterophila nana (Ule) Kriebel \& M.J.R.Rocha

Acisanthera erecta J.St.-Hil

Noterophila rosulans (Huber) Kriebel \& M.J.R.Rocha

Rostranthera tetraptera (Cogn.) M.J.R.Rocha \&

P.J.F.Guim.

Meriania hoyosii (Wurdack) Almeda \& Penneys

Miconia bracteosa (Naudin) Michelang.

Miconia anaectocalyx Michelang.

Miconia manarae (Wurdack) Michelang.

Ernestia rubra Pulle

Huberia capixaba (R.Goldenb. \& Reginato) Bochorny \& Michelang.

Huberia capixaba (R.Goldenb. \& Reginato) Bochorny \& Michelang.

Huberia cordifolia (Cogn.) Bochorny \& R.Goldenb.

Huberia corymbosa (Cogn.) Bochorny \& R.Goldenb.

Huberia edmundoi (Brade) Bochorny \& R.Goldenb.

Huberia hirsuta Bochorny \& R.Goldenb.

Huberia glutinosa (Cogn.) Bochorny \& R.Goldenb.

Huberia huberioides (Brade) Bochorny \& R.Goldenb.

Huberia insignis (Cham.) Bochorny \& R.Goldenb.

Huberia limae (Brade) Bochorny \& R.Goldenb.

Huberia lumiarensis (Bochorny, Michelang. \&

R.Goldenb.) Bochorny

Huberia magdalenensis (Brade) Bochorny \& R.Goldenb.

Huberia mestrealvarensis (D.T.Iglesias \& R.Goldenb.)

Bochorny \& Michelang.

Huberia mourae (Cogn.) Bochorny \& R.Goldenb.

Huberia organensis (Saldanha \& Cogn.) Bochorny \&

R.Goldenb.

Huberia minutifolia Bochorny \& R.Goldenb.

Huberia souzalimae (Brade) Bochorny \& R.Goldenb. 
Calycogonium angulatum Griseb.

Calycogonium apiculatum Urb. \& Ekman

Calycogonium apleurum (Urb. \& Ekman) Judd \&

Skean

Calycogonium bairdianum Skean, Judd, Clase \&

Peguero

Calycogonium bissei Becquer

Calycogonium brevifolium Urb. \& Ekman

Calycogonium calycopteris (Rich.) Urb

Calycogonium clidemioides Griseb.

Calycogonium cocoense Alain

Calycogonium domatiatum Urb. \& Ekman

Calycogonium ekmanii Urb.

Calycogonium ellipticum C.Wright

Calycogonium floribundum Borhidi

Calycogonium formonense Judd, Skean \& Clase

Calycogonium glabratum (Sw.) DC.

Calycogonium grisebachii Triana

Calycogonium hispidulum Cogn

Calycogonium impressum Urb. \& Ekman

Calycogonium lanceolatum Griseb.

Calycogonium lindenianum Naudin

Calycogonium maculatum Urb. \& Ekman

Calycogonium microphyllum C.Wright

Calycogonium moanum (Borhidi \& O.Muñiz)

Borhidi \& O.Muñiz

Calycogonium plicatum Griseb.

Calycogonium pseudofloribundum Becquer

Calycogonium reticulatum (Cogn.) Judd \& Skean

Calycogonium revolutum Alain

Calycogonium rhamnoideum Naudin

Calycogonium rhomboideum Urb. \& Ekman

Calycogonium rosmarinifolium Griseb.

Calycogonium saxicola Britton \& P. Wilson

Calycogonium squamulosum Cogn.

Calycogonium susannae Borhidi

Calycogonium tetragonolobum (Cogn.) Judd \&

Skean

Calycogonium torbecianum Urb. \& Ekman

Calycogonium turbinatum Urb. \& Ekman

Catocoryne linnaeoides Hook.f.
Miconia angulata (Griseb.) M. Gómez

Miconia rufistellata Judd \& Majure

Miconia apleura (Urb. \& Ekman) Judd \& Becquer

Miconia bairdiana ((Skean, Judd, Clase \& Peguero)

Skean \& Judd

Miconia bissei (Becquer) Bécquer \& Michelang

Miconia brevifolia (Urb. \& Ekman) Skean \& Judd

Miconia calycopteris (Rich.) Judd, Bécquer \& Majure

Miconia baracoana M. Gómez

Miconia liogeri Bécquer \& Michelang

Miconia domatiata (Urb. \& Ekman) Skean \& Judd

Miconia erikmaniana Skean \& Judd

Miconia ellipitica (C.Wright) M. Gomez

Miconia cupeyalensis Bécquer \& Michelang

Miconia formonensis (Judd, Skean \& Clase) Judd,

Bécquer \& Majure

Miconia glabrifolia Skean, Judd, Majure \& Bécquer

Miconia grisebachiana Bécquer \& Michelang

Miconia hispidula (Cogn.) Judd, Bécquer \& Majure

Miconia impressinervis Skean \& Judd

Miconia secundo-lanceolata (Griseb.) M. Gómez

Miconia lindeniana (Naudin) M. Gómez

Miconia maculata (Urb. \& Ekman) Judd, Bécquer \& Majure

Miconia microphylla (C.Wright) M. Gómez

Miconia moana (Borhidi \& O.Muñiz) Bécquer \&

Michelang

Miconia plicata (Griseb.) M. Gómez

Miconia pseudofloribunda (Becquer) Bécquer \&

Michelang

Miconia reticulato-venosa Judd, Bécquer \& Majure

Miconia perezii (Alain) Bécquer \& Michelang

Miconia vulcanidomatia Bécquer \& Skean

Miconia rhomboidea ((Urb. \& Ekman)) Skean \& Judd

Miconia rosmarinifolia (Griseb.) M. Gómez

Miconia karsticola Judd, Bécquer, Skean, \& Majure

Henriettea squamulosa (Cogn.) Judd

Miconia susannae (Borhidi) Bécquer \& Michelang

Miconia tetragonoloba (Cogn.) Judd, Bécquer \& Majure

Miconia torbeciana ((Urb. \& Ekman)) Skean \& Judd

Miconia turbinata ((Urb. \& Ekman)) Skean \& Judd

Miconia linnaeoides (Hook. F.) Michelang. 
Centronia brachycera (Naudin) Triana

Centronia crassiramis (Naudin) Triana

Centronia grandiflora Standl.

Centronia haemantha (Planch. \& Lindl.) Triana

Centronia mutabilis Gleason

Centronia mutisii (Bonpl.) Triana

Centronia phlomoides Triana

Charianthus alpinus (Sw.) R.A.Howard

Charianthus corymbosus (Rich.) Cogn.

Charianthus dominicensis Penneys \& Judd

Charianthus grenadensis Penneys \& Judd

Charianthus nodosus (Desr.) Triana

Charianthus purpureus D.Don

Clidemia ablusa Wurdack

Clidemia acostae Wurdack

Clidemia acurensis Wurdack

Clidemia acutifolia Cogn.

Clidemia aguaclarensis Wurdack

Clidemia aguilarii Kriebel \& Almeda

Clidemia allardii Wurdack

Clidemia allenii Almeda

Clidemia almedae Kriebel

Clidemia alternifolia Wurdack

Clidemia ampla Cogn.

Clidemia andersonii Wurdack

Clidemia anisophylla DC.

Clidemia anoriensis L.Uribe

Clidemia aphanantha (Naudin) Sagot

Clidemia asplundii Wurdack

Clidemia atrata Spring

Clidemia attenuata (Naudin) Cogn.

Clidemia aurantiaca Almeda \& Kriebel

Clidemia ayangannensis Wurdack

Clidemia barbata Triana

Clidemia barkleyi Wurdack

Clidemia bernardii Wurdack

Clidemia biolleyana Cogn.

Clidemia biserrata DC.
Meriania brachycera (Naudin) Humberto Mend. \& Fern. Alonso

Meriania crassiramis (Naudin) Wurdack

Meriania grandiflora (Standl.) Almeda

Meriania haemantha (Planch. et Linden) Humberto

Mend. \& Fern. Alonso

Meriania mutabilis (Gleason) Humberto Mend. \& Fern-

Alonso

Meriania mutisii (Bonpl.) Humberto Mend. \& Fern-

Alonso

Meriania phlomoides (Triana) Almeda

Miconia coccinea (Rich.) Judd \& Skean

Miconia corymbosa (Rich.) Judd \& Skean

Miconia dominicensis (Penneys \& Judd) Penneys

Miconia grenadensis (Penneys \& Judd) Penneys

Miconia leblondii Judd \& Skean

Miconia purpurea (D.Don) Judd \& Skean

Miconia ablusa (Wurdack) Michelang.

Miconia acostae (Wurdack) Michelang.

Miconia acurensis (Wurdack) Michelang.

Miconia acutilamina Michelang.

Miconia aguaclarensis (Wurdack) Michelang.

Miconia aguilarii (Kriebel \& Almeda) Gamba \& Almeda

Miconia allardii (Wurdack) Michelang.

Miconia allenii (Almeda) Almeda

Miconia almedae (Kriebel) Michelang.

Miconia alternilamina Michelang.

Miconia amplilamina Michelang.

Miconia willamandersonii Michelang.

Miconia asymmetrica Michelang.

Miconia anoriensis (L.Uribe) Michelang.

Miconia aphanantha (Naudin) Michelang.

Miconia erikasplundii Gamba \& Almeda

Miconia atrata (Spring.) Wawra

Miconia attenuatifolia Michelang.

Miconia aurantiaca (Almeda \& Kriebel) Gamba \&

Almeda

Miconia ayangannensis (Wurdack) Michelang.

Miconia barbipetiolata Michelang.

Miconia barkleyi (Wurdack) Michelang.

Miconia lucianobernardii Michelang.

Miconia biolleyana (Cogn.) Gamba \& Almeda

Miconia biserrata (DC.) Michelang. 
Clidemia bullosa DC.

Clidemia buntingii Wurdack

Clidemia calcarata Cogn. \& Gleason

Clidemia campii Wurdack

Clidemia capillipes (Triana) Cogn.

Clidemia capitata Benth.

Clidemia capitellata (Bonpl.) D.Don

Clidemia caudata Wurdack

Clidemia charadrophylla Tutin

Clidemia chocoensis Wurdack

Clidemia ciliata Pav. ex D.Don

Clidemia clandestina Almeda

Clidemia collina Gleason

Clidemia coloradensis Almeda

Clidemia conglomerata DC.

Clidemia cordata Cogn. ex Britton

Clidemia coronata Gleason

Clidemia costaricensis Cogn. \& Gleason ex Gleason

Clidemia crenulata Gleason

Clidemia crossosepala Griseb.

Clidemia crotonifolia Pilg.

Clidemia cruegeriana Griseb.

Clidemia cuatrecasasii Wurdack

Clidemia cursoris Wurdack

Clidemia cutucuensis Wurdack

Clidemia cymifera Donn. Sm.

Clidemia davidsei Almeda

Clidemia debilis Crueg.

Clidemia densiflora (Standl.) Gleason

Clidemia dentata D.Don

Clidemia diffusa Donn. Sm.

Clidemia diguensis Wurdack

Clidemia dimorphica J.F.Macbr.

Clidemia discolor (Triana) Cogn.

Clidemia domingensis (DC.) Cogn.

Clidemia donnell-smithii Cogn.

Clidemia duidae Gleason

Clidemia ecuadorensis Gleason

Clidemia epibaterium DC.

Clidemia epiphytica (Triana) Cogn.
Miconia bullosa (DC.) Michelang.

Miconia georgebuntingii Michelang.

Miconia neocalcarata Michelang.

Miconia waltercampii Michelang.

Miconia capillipes (Triana) Michelang.

Miconia benthamii (Naudin) Michelang.

Miconia dependens (Pav. ex D. Don) Judd \& Majure

Miconia caudatifolia Michelang.

Miconia charadrophylla (Tutin) Michelang.

Miconia chocoensis (Wurdack) Gamba \& Almeda

Miconia domociliata Michelang.

Miconia clandestina (Almeda) Almeda

Miconia formicocollina Michelang.

Miconia neocoloradensis Almeda

Miconia conglomerata (DC) Michelang.

Miconia neocordata Michelang.

Miconia neocoronata Gamba \& Almeda

Miconia skutchiana Michelang.

Miconia crenulata (Gleason) Michelang.

Miconia crossosepala (Griseb.) Ionta \& Judd

Miconia neocrotonifolia Michelang.

Miconia neocrugeriana Michelang.

Miconia quadridomius Gamba \& Almeda

Miconia cursoris (Wurdack) Michelang.

Miconia disticha Michelang.

Miconia cymifera (Donn. Sm.) Michelang.

Miconia davidsei (Almeda) Almeda

Miconia debilis (Crueg.) Michelang.

Miconia approximata Gamba \& Almeda

Miconia dentata (D. Don) Michelang.

Miconia verapazana Michelang.

Miconia diguensis (Wurdack) Michelang.

Miconia dimorphica (J. F. Macbr.) Michelang.

Miconia purpureoviolacea (Cogn.) Michelang.

Miconia hispaniolica Judd \& Majure

Miconia mesoamericana Michelang.

Miconia duidae (Gleason) Michelang.

Miconia ecuadorensis (Gleason) Michelang.

Miconia epibaterium (DC) Michelang.

Miconia neoepiphyitca Michelang. 
Clidemia erythropogon DC.

Clidemia evanescens Almeda

Clidemia farinasii Wurdack

Clidemia fausta Wurdack

Clidemia fendleri Cogn.

Clidemia fissinervia Gleason

Clidemia flexuosa (Triana) Cogn.

Clidemia fluminensis Baumgratz \& D'El Rei Souza

Clidemia foliosa Gleason

Clidemia folsomii Almeda

Clidemia foreroi Wurdack

Clidemia francavillana Cogn.

Clidemia fraterna Gleason

Clidemia fulva Gleason

Clidemia garciabarrigae Wurdack

Clidemia globuliflora (Cogn.) L.O.Williams

Clidemia graciliflora Huber

Clidemia gracilipes Gleason

Clidemia gracilis Pittier

Clidemia grandifolia Cogn.

Clidemia granvillei Wurdack

Clidemia grisebachii Cogn.

Clidemia guadaloupensis (DC) Griseb.

Clidemia hammelii Almeda

Clidemia heptamera Wurdack

Clidemia heteronervis (Naudin) Wurdack

Clidemia heteroneura (Schrank \& Mart. ex DC.)

Cogn.

Clidemia heterophylla (Desr.) Gleason

Clidemia hirta (L.) D.Don

Clidemia imparilis Wurdack

Clidemia inobsepta Wurdack

Clidemia inopinata Almeda

Clidemia inopogon Mart.

Clidemia intonsa E.Cotton \& W.Meier

Clidemia involucrata DC.

Clidemia japurensis DC.

Clidemia juruensis (Pilg.) Gleason

Clidemia kappleri Cogn.

Clidemia killipii Gleason

Clidemia laevifolia Gleason
Miconia erytrhopogon (DC.) Judd \& Ionta

Miconia evanescens (Almeda) Gamba \& Almeda

Miconia farinasii (Wurdack) Michelang.

Miconia fausta (Wurdack) Michelang.

Miconia rariflora (Bonpl) Michelang.

Miconia fissinervia (Gleason) Michelang.

Miconia flexuosa (Triana) Michelang.

Miconia pleiofluminensis R.Goldenb \& Michelang.

Miconia formicofoliosa Michelang.

Miconia folsomii (Almeda) Almeda

Miconia foreroi (Wurdack) Michelang.

Miconia pseudodebilis Michelang.

Miconia fraterna (Gleason) Michelang.

Miconia crocina Michelang.

Miconia garcia-barrigae (Wurdack) Almeda

Miconia reflexa (Gleason) Michelang.

Miconia graciliflora (Huber) Michelang.

Miconia gracilipes (Gleason) Michelang.

Miconia pseudoalternifolia Michelang.

Miconia badilloi Michelang.

Miconia granvillei (Wurdack) Michelang.

Miconia augustgrisebachii Ionta \& Judd

Miconia guadalupensis (DC.) Judd, Ionta, \& Majure

Miconia hammelii (Almeda) Almeda

Miconia heptamera (Wurdack) Michelang.

Miconia heteronervis (Naudin) Michelang.

Miconia heteroneura (Schrank. et Mart. ex DC.)

Michelang.

Miconia formicoheterophylla Michelang.

Miconia crenata (Vahl.) Michelang.

Miconia imparilis (Wurdack) Michelang.

Miconia inobsepta (Wurdack) Michelang.

Miconia inopinata (Almeda) Almeda

Miconia ionopogon (DC) R.Goldenb.

Miconia intonsa (E.Cotton \& W.Meier) Michelang.

Miconia cephaloides Michelang.

Miconia heteroclita (Naudin) Michelang.

Miconia formicojuruensis Michelang.

Miconia augustkappleri Michelang.

Miconia ellsworthii Michelang.

Miconia laevifolia (Gleason) Michelang. 
Clidemia lanuginosa Almeda

Clidemia laxiflora (Schltdl.) Walp. ex Naudin

Clidemia leucandra C.Wright ex Griseb.

Clidemia leucandra var. divaricata C.Wright ex

Griseb.

Clidemia linearis (Gleason) Wurdack

Clidemia longifolia Gleason

Clidemia longipedunculata E.Cotton

Clidemia macropetala R.O. Williams

Clidemia marahuacensis Wurdack

Clidemia matudae L.O.Williams

Clidemia micrantha Sagot

Clidemia microthyrsa R.O.Williams

Clidemia monantha L.O.Williams

Clidemia morichensis Wurdack

Clidemia mortoniana Standl.

Clidemia myrmecina Gleason

Clidemia neblinae Wurdack

Clidemia novemnervia (DC.) Triana

Clidemia obliqua Griseb. ex Cogn.

Clidemia oblonga Gleason

Clidemia octona (Bonpl.) L.O.Williams

Clidemia ombrophila Gleason

Clidemia ostentata Wurdack

Clidemia ostrina Gleason

Clidemia oxyura (Naudin) Triana

Clidemia pakaraimae Wurdack

Clidemia pectinata Almeda

Clidemia petiolaris (Schltdl. \& Cham.) Schltdl. ex

Triana

Clidemia petiolata DC.

Clidemia pilosa Pav. ex D.Don

Clidemia piperifolia Gleason

Clidemia plumosa (Desr.) DC.

Clidemia pterosepala (Urb.) Alain

Clidemia pubescens Gleason

Clidemia purpurea Pav. ex Don

Clidemia pustulata DC.

Clidemia pycnantha Urb. \& Ekman

Clidemia pycnaster Tutin

Clidemia quinquenervia (Mill.) Almeda
Miconia neolanuginosa Almeda

Miconia laxiflora (Schltdl.) Michelang.

Miconia leucandra (C.Wright ex Griseb.) Judd \& Ionta

Miconia divaricatiflora Judd \& Becquer

Miconia linearis (Gleason) Michelang.

Miconia adventitia Michelang.

Miconia cottoniana Michelang.

Miconia macropetala Michelang.

Miconia marahuacencis (Wurdack) Michelang.

Miconia matudae (L. O. Wms.) Michelang.

Miconia drosera (Sagot) Michelang.

Miconia microthyrsa (L.O.Wms.) Michelang.

Miconia monantha (L. O. Wms.) Michelang.

Miconia morichensis (Wurdack) Michelang.

Miconia mortoniana (Standl.) Michelang.

Miconia myrmecina (Gleason) Michelang.

Miconia neblinae (Wurdack) Michelang.

Miconia bullatifolia Michelang.

Miconia alaticualis Michelang.

Miconia oblonga (Gleason) Michelang.

Miconia octona (Bonpl.) Judd \& Majure

Miconia silviphila Michelang.

Miconia ostentata (Wurdack) Michelang.

Miconia ostrina (Gleason) Michelang.

Miconia oxyura (Naudin) Michelang.

Miconia pakaraimae (Wurdack) Michelang.

Miconia neopectinata Almeda

Miconia petiolaris (Schltdl. \& Cham.) Michelang.

Miconia petiolata (DC.) Michelang.

Miconia neopilosa Michelang.

Miconia piperilamina Michelang.

Miconia berteroi (DC) Judd \& Ionta

Miconia pterosepala Urb.

Miconia pubescens (Gleason) Michelang.

Miconia atropurpurea Gamba \& Almeda

Miconia fenestrata (Seem.) Michelang.

Miconia pycnantha (Urb. \& Ekman) Ionta, Judd \& Skean

Miconia pycnaster (Tutin) Michelang.

Miconia quinquenervia (Mill.) Gamba \& Almeda 
Clidemia radicans Cogn.

Clidemia reitziana Cogn. \& Gleason ex Gleason

Clidemia repens Triana

Clidemia retropila DC

Clidemia rodriguezii Almeda

Clidemia rubra (Aubl.) Mart.

Clidemia rubrinervis (Naudin) Cogn.

Clidemia rubripila Cogn.

Clidemia ruddae Wurdack

Clidemia saltuensis Wurdack

Clidemia sandwithii Wurdack

Clidemia saulensis Wurdack

Clidemia scalpta (Vent.) F. S. Axelrod

Clidemia scopulina (Brandegee) L.O.Williams

Clidemia semijuga (Gleason) Wurdack

Clidemia septuplinervia Cogn.

Clidemia sericea D.Don

Clidemia serpens (Triana) Cogn.

Clidemia sessiliflora (Naudin) Cogn.

Clidemia setosa (Triana) Gleason

Clidemia siapensis Wurdack

Clidemia silvicola Gleason

Clidemia simpsonii Wurdack

Clidemia smithii Gleason

Clidemia spectabilis Gleason

Clidemia sprucei Gleason

Clidemia stellipilis (Gleason) Wurdack

Clidemia steyermarkii Wurdack

Clidemia strigillosa (Sw.) DC.

Clidemia submontana Rose ex Gleason

Clidemia subpeltata Kriebel \& Almeda

Clidemia swartzii Griseb.

Clidemia taurina Gleason

Clidemia tenebrosa Almeda

Clidemia tepuiensis Wurdack

Clidemia tetrapetala Almeda

Clidemia tetraptera Cogn.

Clidemia tillettii Wurdack

Clidemia tococoidea (DC.) Gleason

Clidemia trichosantha Almeda
Miconia radicans (Cogn.) Gamba \& Almeda Miconia reitziana (Cogn. \& Gleason ex Gleason) Gamba \& Almeda

Miconia repens (Triana) Michelang.

Miconia retropila (DC.) Ocampo \& Almeda

Miconia rodriguezii (Almeda) Almeda

Miconia rubra (Aubl.) Mabb.

Miconia rubrinervis (Naudin) Judd \& Becquer

Miconia rubripila (Cogn.) Judd \& Ionta

Miconia ruddae (Wurdack) Michelang.

Miconia chichirivichensis Michelang.

Miconia sandwithii (Wurdack) Michelang.

Miconia saulensis (Wurdack) Michelang.

Miconia scalpta (Vent.) Ionta, Judd \& Skean

Miconia scopulina (Brand.) Michelang.

Miconia semijuga (Gleason) Michelang.

Miconia trichocalyx (Pittier) Michelang. \& Judd

Miconia sericea (D. Don) Michelang.

Miconia serpens (Triana) Michelang.

Miconia sessiliflora (Naudin) Michelang.

Miconia formicosetosa Michelang.

Miconia siapensis (Wurdack) Michelang.

Miconia silvicola (Gleason) Michelang.

Miconia simpsonii (Wurdack) Michelang.

Miconia albertsmithii Michelang.

Miconia spectabilis (Gleason) Michelang.

Miconia richardsprucei Michelang.

Miconia sororopana Michelang.

Miconia julianii Michelang.

Miconia strigillosa (Sw.) Judd \& Ionta

Miconia submontana (Rose ex Gleason) Michelang.

Miconia subpeltata (Kriebel \& Almeda) Almeda

Miconia pilosa ( $\mathrm{Sw}$ ) Judd \& Ionta

Miconia taurina (Gleason) Michelang.

Miconia tenebrosa (Almeda) Almeda

Miconia nebulensis Michelang.

Miconia tetrapetala (Almeda) Almeda

Miconia tetraptera (Cogn.) Ionta, Judd \& Skean

Miconia stephentillettii Michelang.

Miconia tococoidea (Gleason) Michelang.

Miconia trichosantha (Almeda) Almeda 
Clidemia trichotoma C.Wright ex Griseb.

Clidemia trinitensis (Crueg.) Griseb.

Clidemia tuerckheimii (Donn. Sm.) Gleason

Clidemia ulei Pilg.

Clidemia umbrosa (Sw.) Cogn.

Clidemia urceolata DC.

Clidemia uribei Wurdack

Clidemia urticoides Pilg.

Clidemia utleyana Almeda

Clidemia vallicola Gleason

Clidemia variifolia Wurdack

Clidemia vegaensis Cogn.

Clidemia venosa (Gleason) Wurdack

Clidemia ventricosa Almeda

Clidemia verruculosa Wurdack

Comolia ayangannae Wurdack

Comolia coriacea Gleason

Comolia edmundoi Brade

Comolia lanceiflora (DC.) Triana

Comolia montana Gleason

Comolia sertularia (DC.) Triana

Comolia sessilis (Spreng.) Triana

Comolia stenodon Triana

Comolia vernicosa (Benth.) Triana

Conostegia apiculata Wurdack

Conostegia arborea (Schltdl.) Steud.

Conostegia attenuata Triana

Conostegia balbisiana Ser. ex DC.

Conostegia bigibbosa Cogn.

Conostegia bracteata Triana

Conostegia brenesii Standl.

Conostegia caelestis Standl.

Conostegia centronioides Markgr.

Conostegia centronioides var. lancifolia Markgr.

Conostegia chiriquensis Gleason

Conostegia cinnamomea (Beurl.) Wurdack

Conostegia cuatrecasii Gleason

Conostegia dentata Triana

Conostegia extinctoria (Bonpl.) D.Don ex DC.
Miconia hirticalyx Judd \& Becquer

Miconia trinitensis (Crueg) Michelang.

Miconia hanstuerckheimii Michelang.

Miconia ernstulei Michelang.

Miconia sciaphila Judd \& Ionta

Miconia neourceolata Michelang.

Miconia lorenzouribei Michelang.

Miconia urticilamina Michelang.

Miconia utleyana (Almeda) Almeda

Miconia vallicola (Gleason) Michelang.

Miconia variifolia (Wurdack) Michelang.

Miconia vegaensis (Cogn.) Judd, Bécquer \& Majure

Miconia venosa (Gleason) Michelang.

Miconia ventricosa (Almeda) Almeda

Miconia verruculosa (Wurdack) P.Jørg. \& C.Ulloa

Acisanthera ayangannae

(Wurdack)M.J.R.Rocha\&P.J.F.Guim.,

Comoliopsis coriacea (Gleason) M.J.R.Rocha \&

P.J.F.Guim.

Fritzschia edmundoi (Brade) M.J.R.Rocha \& P.J.F.Guim.

Fritzschia lanceiflora (DC.) M.J.R.Rocha \& P.J.F.Guim.

Comoliopsis montana (Gleason) M.J.R.Rocha \&

P.J.F.Guim.

Fritzschia sertularia (DC.) M.J.R.Rocha \& P.J.F.Guim.

Fritzschia sessilis (Spreng.) M.J.R.Rocha \& P.J.F.Guim.

Fritzschia stenodon (Triana) M.J.R.Rocha \& P.J.F.Guim.

Leiostegia vernicosa Benth.

Miconia conoapiculata Michelang.

Miconia galeottii (Naudin) Michelang.

Miconia conoattenuata Michelang.

Miconia balbisiana (Ser. ex Dc.) Michelang.

Miconia bigibbosa (Cogn.) Michelang.

Miconia conobracteata Michelang.

Miconia conobrenesii Michelang.

Miconia caelestis (Standl.) Michelang.

Miconia conocentronioides Michelang.

Miconia conolancifolia Michelang.

Miconia conochiriquensis Michelang.

Miconia cinnamomea Beurl.

Miconia conocuatrecasii Michelang.

Miconia conodentata Michelang.

Miconia extinctoria (Bonpl.) Michelang. 
Conostegia fragrantissima Almeda

Conostegia hirtella Cogn.

Conostegia icosandra (Sw. ex Wikstr.) Urb.

Conostegia inusitata Wurdack

Conostegia jaliscana Standl.

Conostegia lasiopoda Benth.

Conostegia lindenii Cogn.

Conostegia macrantha O.Berg ex Triana

Conostegia micrantha Standl.

Conostegia montana (Sw.) D.Don ex DC.

Conostegia monteleagreana Cogn.

Conostegia muriculata Almeda

Conostegia oerstediana O.Berg ex Triana

Conostegia ortizae Kriebel

Conostegia pittieri Cogn.

Conostegia pittieri Cogn. ex T.Durand

Conostegia plumosa L.O.Williams

Conostegia polyandra Benth.

Conostegia procera (Sw.) D.Don

Conostegia pyxidata Proctor

Conostegia rhodopetala Donn. Sm.

Conostegia rubiginosa Gleason

Conostegia rufescens Naudin

Conostegia setifera Standl.

Conostegia setosa Triana

Conostegia speciosa Naudin

Conostegia subcrustulata (Beurl.) Triana

Conostegia superba Naudin

Conostegia tenuifolia Donn. Sm.

Conostegia volcanalis Standl. \& Steyerm.

Conostegia vulcanicola Donn. Sm.

Conostegia xalapensis (Bonpl.) D.Don ex DC.

Diolena agrimonioides Triana

Diolena allardii Wurdack

Diolena amazonica Pilg.

Diolena auriculata Triana

Diolena calciphila Standl. \& Steyerm.

Diolena campii Wurdack

Diolena hygrophylla Naudin

Diolena lanceolata Gleason
Miconia fragrantissima (Almeda) Michelang. \& Almeda

Miconia conohirtella Michelang.

Miconia subhirsuta (DC.) M. Gomez

Miconia inusitata (Wurdack) Michelang. \& R.Goldenb.

Miconia jaliscana (Standl.) Michelang.

Miconia lasiopoda (Benth.) Michelang.

Miconia lomensis (Urb.) Michelang.

Miconia conomacrantha Michelang.

Miconia conomicrantha Michelang.

Miconia cooperi (Cogn) Michelang.

Miconia monteleagreana (Cogn) Michelang.

Miconia muriculata (Almeda) Michelang. \& Almeda

Miconia oerstediana (O. Berg ex Triana) Michelang.

Miconia ortizae (Kriebel) Michelang.

Miconia durandii Michelang. \& P.Jørg

Miconia donnell-smithii (Cogn.) Michelang.

Miconia conoplumosa Michelang.

Miconia rupicola Gleason

Miconia gloriosa (Macfad) Michelang.

Miconia pyxidata (Proctor) Michelang.

Miconia rhodopetala (Donn-Sm.) Michelang.

Miconia conorubiginosa Michelang.

Miconia conorufescens Michelang.

Miconia conosetifera Michelang.

Miconia conosetosa Michelang.

Miconia conospeciosa Michelang.

Miconia subcrustulata Beurl.

Miconia baillonii M. Gomez

Miconia tenuifolia (Donn. Sm.) Michelang.

Miconia volcanalis (Standl. \& Steyerm.) Michelang.

Miconia vulcanicola (Donn. Sm.) Michelang.

Miconia xalapensis (Bonpl.) M. Gomez

Triolena agrimonioides (Triana) Almeda \& Alvear

Triolena allardii (Wurdack) Wurdack

Triolena amazonica (Pilg.) Wurdack

Triolena auriculata (Triana) Almeda \& Alvear Triolena calciphila (Standl. \& Steyerm.) Standl. \&

L.O.Williams

Triolena campii (Wurdack) Wurdack

Triolena hygrophylla (Naudin) L.O.Williams

Triolena lanceolata (Gleason) Almeda \& Alvear 
Diolena obliqua Triana

Diolena pileoides Triana

Diolena pluvialis Wurdack

Diolena purpurea Gleason

Diolena spicata Triana

Diolena stenophylla Standl. \& Steyerm.

Diplarpea paleacea Triana

Dolichoura kollmannii R.Goldenb. \& R. Tavares

Dolichoura spiritusanctensis Brade

Ernestia cordifolia O. Berg ex Triana

Ernestia glandulosa Gleason

Ernestia pullei Gleason

Ernestia subglabra Wurdack

Henriettea hotteana (Urb. \& Ekman) Alain

Henriettea hotteana (Urb. \& Ekman) Alain

Henriettella acunae Alain

Henriettella aggregata (D.Don) Triana

Henriettella barkeri Urb. \& Ekman

Henriettella boliviensis Cogn.

Henriettella bracteosa Wurdack

Henriettella caudata Gleason

Henriettella ciliata Urb. \& Ekman

Henriettella cuabae (Urb.) Bisse

Henriettella cuneata (Standl.) Gleason

Henriettella duckeana Hoehne

Henriettella ekmanii Urb.

Henriettella fascicularis (Sw.) C.Wright

Henriettella fissanthera Gleason

Henriettella flavescens (Aubl.) Triana

Henriettella gibberosa Urb.

Henriettella glabra (Vell.) Cogn.

Henriettella goudotiana Naudin

Henriettella heteroneura Gleason

Henriettella hondurensis Wurdack
Triolena obliqua (Triana) Wurdack

Triolena pileoides (Triana) Wurdack

Triolena pluvialis (Wurdack) Wurdack

Triolena purpurea (Gleason) Almeda \& Alvear

Triolena spicata (Triana) L.O.Williams

Triolena stenophylla (Standl. \& Steyerm.) Standl. \&

L.O.Williams

Triolena paleacea (Triana) Almeda \& Alvear, Huberia kollmannii (R.Goldenb. \& R.Tav.) Bochorny \& Michelang.

Huberia bradeana Bochorny \& R.Goldenb.

Pseudoernestia cordifolia (O.Berg ex Triana) Krasser

Pseudoernestia glandulosa (Gleason) M.J.R.Rocha \&

P.J.F.Guim.

Appendicularia pullei (Gleason) M.J.R.Rocha \&

P.J.F.Guim

Appendicularia subglabra (Wurdack) M.J.R.Rocha \&

P.J.F.Guim.

Miconia hotteana (Urb. \& Ekman) Judd \& Ionta

Miconia hotteana (Urb. \& Ekman) Judd \& Ionta

Henriettea acunae (Alain) Alain

Henriettea aggregata (D.Don) J.F.Macbr.

Henriettea barkeri (Urb. \& Ekman) Alain

Henriettea boliviensis (Cogn.) Penneys, Michelangeli, Judd \& Almeda

Henriettea bracteosa (Wurdack) Penneys, Michelangeli, Judd \& Almeda

Henriettea caudata (Gleason) Penneys, Michelangeli, Judd \& Almeda

Henriettea ciliata (Urb. \& Ekman) Alain

Henriettea cuabae (Urb.) Borhidi

Henriettea cuneata (Standl.) L.O.Williams

Henriettea duckeana (Hoehne) Penneys, Michelangeli, Judd \& Almeda

Henriettea ekmanii (Urb.) Alain

Henriettea fascicularis (Sw.) M.Gómez

Henriettea fissanthera (Gleason) Penneys, Michelangeli, Judd \& Almeda

Henriettea flavescens (Aubl.) Baill.

Henriettea gibberosa (Urb.) Alain

Henriettea glabra (Vell.) Penneys, Michelangeli, Judd \& Almeda

Henriettea goudotiana (Naudin) Penneys, Michelangeli, Judd \& Almeda

Henriettea heteroneura (Gleason) Penneys, Michelangeli, Judd \& Almeda

Henriettea hondurensis (Wurdack) Penneys,

Michelangeli, Judd \& Almeda 
Henriettella ininiensis Wurdack

Henriettella lateriflora (Vahl) Triana

Henriettella lawrencei Gleason

Henriettella loretensis Gleason

Henriettella lundellii Wurdack

Henriettella macfadyenii Triana

Henriettella maguirei Wurdack

Henriettella manarae Wurdack

Henriettella megaloclada Urb. \& Ekman

Henriettella membranifolia Cogn.

Henriettella odorata Markgr.

Henriettella ovata Cogn.

Henriettella prancei Wurdack

Henriettella reflexa Urb. \& Ekman

Henriettella rimosa Wurdack

Henriettella seemannii Naudin

Henriettella sessilifolia (L.) Triana

Henriettella squamata Alain

Henriettella steyermarkii Wurdack

Henriettella sylvestris Gleason

Henriettella tachirensis Wurdack

Henriettella tobagensis Wurdack

Henriettella tovarensis Cogn.

Henriettella trachyphylla Triana

Henriettella triflora (Vahl) Triana

Henriettella tuberculosa Donn. Sm.

Henriettella verrucosa Triana

Huilaea calyptrata Penneys \& Morales-P.

Huilaea ecuadorensis Wurdack

Huilaea kirkbridei Wurdack

Huilaea macrocarpa L.Uribe

Huilaea minor (L.Uribe) Lozano \& N.Ruiz-R.

Huilaea mutisiana L.Uribe
Henriettea ininiensis (Wurdack) Penneys, Michelangeli, Judd \& Almeda

Henriettea lateriflora (Vahl) R.A.Howard \& E.A.Kellogg Henriettea lawrancei (Gleason) Penneys, Michelangeli, Judd \& Almeda

Henriettea loretensis (Gleason) J.F.Macbr. Henriettea lundellii (Wurdack) Penneys, Michelangeli, Judd \& Almeda

Henriettea macfadyenii (Triana) Alain Henriettea maguirei (Wurdack) Penneys, Michelangeli, Judd \& Almeda

Henriettea manarae (Wurdack) Penneys, Michelangeli, Judd \& Almeda

Henriettea megaloclada (Urb. \& Ekman) Alain

Henriettea membranifolia (Cogn.) Alain

Henriettea odorata (Markgr.) Penneys, Michelangeli,

Judd \& Almeda

Henriettea ovata (Cogn.) Penneys, Michelangeli, Judd \& Almeda

Henriettea prancei (Wurdack) Penneys, Michelangeli, Judd \& Almeda

Henriettea reflexa (Urb. \& Ekman) Alain

Henriettea rimosa (Wurdack) Penneys, Michelangeli, Judd \& Almeda

Henriettea seemannii (Naudin) L.O.Williams

Henriettea sessilifolia (L.) Alain

Henriettea squamata (Alain) Alain

Henriettea steyermarkii (Wurdack) Penneys,

Michelangeli, Judd \& Almeda

Henriettea sylvestris (Gleason) J.F.Macbr.

Henriettea tachirensis (Wurdack) Penneys, Michelangeli, Judd \& Almeda

Henriettea tobagensis (Wurdack) Penneys, Michelangeli, Judd \& Almeda

Henriettea tovarensis (Wurdack) Penneys, Michelangeli, Judd \& Almeda

Henriettea trachyphylla (Triana) Penneys, Michelangeli, Judd \& Almeda

Henriettea triflora (Vahl) Alain

Henriettea tuberculosa (Donn. Sm.) L.O.Williams

Henriettea verrucosa (Triana) J.F.Macbr.

Chalybea calyptrata (Penneys \& Morales-P.) Penneys \& Morales-P.,

Chalybea ecuadorensis (Wurdack) Morales-P. \& Penneys

Chalybea kirkbridei (Wurdack) Morales-P. \& Penneys

Chalybea macrocarpa (L.Uribe) Morales-P. \& Penneys

Chalybea minor (L.Uribe) Morales-P. \& Penneys

Chalybea mutisiana L.Uribe) Morales-P. \& Penneys 
Huilaea occidentalis Lozano \& N.Ruiz-R.

Huilaea penduliflora Wurdack

Killipia latifolia Wurdack

Killipia pedunculata Gleason

Killipia quadrangularis Gleason

Killipia rotundifolia Wurdack

Killipia verticalis N.Ruiz-R.

Leandra acuminata Cogn.

Leandra acutiflora (Naudin) Cogn.

Leandra adamantinensis Brade

Leandra adenothrix Cogn.

Leandra agrestis (Aubl.) Raddi

Leandra alpestris (Gardn.) Cogn.

Leandra altomacaensis Baumgratz \& D'El Rei Souza

Leandra amplexicaulis DC.

Leandra aptera (DC.) Cogn.

Leandra aristigera (Naudin) Cogn.

Leandra aspera Cogn.

Leandra atrata Cogn.

Leandra atroviridis Cogn.

Leandra aurea (Cham.) Cogn.

Leandra australis (Cham.) Cogn.

Leandra balansaei Cogn.

Leandra barbinervis (Cham. ex Triana) Cogn.

Leandra blanchetiana (Triana) Cogn.

Leandra boissieriana Cogn.

Leandra boliviensis Cogn.

Leandra brackenridgei (A. Gray) Cogn.

Leandra breviflora Cogn.

Leandra calvescens (O. Berg ex Triana) Cogn.

Leandra camporum Brade

Leandra cancellata Cogn.

Leandra candelabrum (J.F.Macbr.) Wurdack

Leandra capilliflora (Naudin) Reginato

Leandra capitata Reginato

Leandra caquetana Sprague

Leandra caquetensis Gleason

Leandra carassana (DC.) Cogn.

Leandra catharinensis Cogn.
Chalybea occidentalis Lozano \& N.Ruiz-R) Morales-P. \& Penneys.

Chalybea penduliflora Wurdack) Morales-P. \& Penneys

Miconia jamesluteynii Michelang.

Miconia aurantipetala Michelang.

Miconia quadricaulis Michelang.

Miconia roseopetala Michelang.

Miconia verticalis (N. Ruiz-R.) Michelang.

Miconia leacuminata R.Goldenb.

Miconia acutiflora (Naudin) R.Goldenb.

Miconia adamantinensis (Brade) R.Goldenb.

Miconia adenothrix (Cogn.) R.Goldenb.

Miconia agrestis (Aubl. ) Raddi

Miconia lealpestris R.Goldenb.

Miconia altomacaensis (Baumgratz \& D'El Rei Souza)

R.Goldenb.

Miconia pectinata (Cogn.) R.Goldenb.

Miconia aptera (DC.) R.Goldenb.

Miconia aristigera (Naudin) R.Goldenb. \& Michelang.

Miconia aspera (Cogn.) R.Goldenb.

Miconia leatrata R.Goldenb.

Miconia atroviridis (Cogn.) R.Goldenb.

Miconia auricoma (Spring. ex Mart.) R.Goldenb.

Miconia australis (Cham.) R.Goldenb.

Miconia balansae (Cogn.) R.Goldenb.

Miconia trauninensis (Cogn.) R.Goldenb.

Miconia leablanchetiana R.Goldenb.

Miconia pierreboissieriana R.Goldenb.

Miconia yungasensis R.Goldenb. \& Michelang.

Miconia brackenridgei (A.Gray) R.Goldenb.

Miconia breviflora (Cogn.) R.Goldenb.

Miconia leacalvescens R.Goldenb.

Miconia camporum (Brade) R.Goldenb.

Miconia cancellata (Cogn.) R.Goldenb.

Miconia candelabrum (J.F.Macbr.) R.Goldenb. \&

Michelang.

Miconia capilliflora (Naudin) R.Goldenb.

Miconia leacapitata R.Goldenb.

Miconia secuncaquetana G. Ocampo \& Almeda

Miconia caquetensis (Gleason) G. Ocampo \& Almeda

Miconia sublanata (Cogn.) R.Goldenb.

Miconia catharinensis (Cogn.) R.Goldenb. 
Leandra chaetocalyx (A.Gray) Cogn

Leandra chaetodon (DC.) Cogn.

Leandra cinerea (Griseb.) Cogn.

Leandra circumscissa Cogn.

Leandra clidemioides (Naudin) Wurdack

Leandra coadunata Wurdack

Leandra cogniauxii (D'El Rei Souza) Reginato

Leandra collina Wurdack

Leandra confusa Cogn.

Leandra cordifolia (Naud.) Cogn.

Leandra cordigera (Triana) Cogn.

Leandra coriacea Cogn.

Leandra cornoides (Schltdl. \& Cham.) Cogn.

Leandra cremersii Wurdack

Leandra crenata (Pav. ex D.Don) Cogn.

Leandra cristata Reginato \& R.Goldenb.

Leandra cuatrecasasii Wurdack

Leandra cuneata (Mart.) Cogn.

Leandra debilis (Naudin) Cogn.

Leandra deflexa (O. Berg. ex Triana) Cogn.

Leandra dendroides (Naudin) Cogn.

Leandra dentata Cogn.

Leandra dichotoma (Pav. ex D.Don) Cogn.

Leandra diffusa Cogn.

Leandra dispar (Gardner) Cogn.

Leandra divaricata (Naudin) Cogn.

Leandra dolichantha (Naudin) Cogn.

Leandra dubia DC.

Leandra echinata Cogn.

Leandra edentula Gleason

Leandra eichleri Cogn.

Leandra eriocalyx Cogn.

Leandra erostrata (DC.) Cogn.

Leandra fallacissima Markgr.

Leandra fallax (Cham.) Cogn.

Leandra fastigiata Cogn.

Leandra flavescens Cogn.

Leandra floribunda (Naudin) Cogn .

Leandra fluminensis Cogn.
Miconia chaetocalyx (A.Gray) R.Goldenb.

Miconia chaetodonta R.Goldenb. \& Michelang.

Miconia cubacinerea Majure \& Judd

Miconia circumscissa (Cogn.) R.Goldenb.

Miconia stenopetala Griseb.

Miconia coadunata (Wurdack) R.Goldenb.

Miconia cogniauxii (Glaz. Ex. Del Rei Souza)

R.Goldenb.

Miconia leacollina R.Goldenb.

Miconia leaconfusa R.Goldenb.

Miconia leacordifolia R.Goldenb.

Miconia cordigera (Triana) R.Goldenb.

Miconia leacoriacea R.Goldenb.

Miconia cornoides (Schltdl. \& Cham.) Almeda

Miconia cremersii (Wurdack) G. Ocampo \& Almeda

Miconia leacrenata R.Goldenb. \& Michelang.

Miconia cristata (Reginato \& R.Goldenb.) R.Goldenb.

Miconia josecuatrecasasii R.Goldenb. \& Michelang.

Miconia cuneatissima R.Goldenb. \& Michelang.

Miconia leadebilis R.Goldenb.

Miconia deflexa (Triana) R.Goldenb.

Miconia dendroides (Naudin) R.Goldenb.

Miconia leadentata R.Goldenb.

Miconia sulcicaulis (Poepp. ex Naudin) G. Ocampo \&

Almeda

Miconia diffusa (Cogn.) R.Goldenb.

Miconia leadispar R.Goldenb.

Miconia secundivaricata G. Ocampo \& Almeda

Miconia dolichantha (Naudin) R.Goldenb.

Miconia dubia (DC.) R.Goldenb.

Miconia leaechinata R.Goldenb.

Miconia edentula (Gleason) Michelang. \& R.Goldenb.

Miconia leaeichleri Michelang. \& R.Goldenb.

Miconia leaeriocalyx R.Goldenb.

Miconia erostrata (DC.) R.Goldenb.

Miconia fallacissima (Markgr) R.Goldenb.

Miconia leafallax R.Goldenb.

Miconia fastigiata (Cogn.) R.Goldenb.

Miconia leaflavescens R.Goldenb.

Miconia leafloribunda R.Goldenb.

Miconia leafluminensis R.Goldenb. 
Leandra fontanae Reginato \& R.Goldenb.

Leandra foveolata (DC.) Cogn.

Leandra francavillana Cogn.

Leandra freyreissii Cogn.

Leandra glabrata (Bunb.) Cogn.

Leandra glandulifera (Triana) Cogn.

Leandra glazioviana Cogn.

Leandra gorzulae Wurdack

Leandra gracilis Cogn.

Leandra granatensis Gleason

Leandra grandifolia Cogn.

Leandra grayana Cogn.

Leandra gynoverrucosa Reginato

Leandra hatschbachii Brade

Leandra herincquiana Cogn.

Leandra hermogenesii Baumgratz \& D'El Rei Souza

Leandra heteroporata Reginato

Leandra hirtella Cogn.

Leandra horrida Cogn.

Leandra humilis (Cogn.) Wurdack

Leandra hybophylla (Urb.) Alain

Leandra inaequalifolia (DC) Cogn.

Leandra inaequidens (Urb. \& Ekm.) Judd \& Skean

Leandra intermedia (DC) Cogn.

Leandra ionopogon (Mart.) Cogn.

Leandra itatiaiae (Wawra) Cogn.

Leandra kleinii Brade

Leandra krugiana (Cogn.) Judd \& Skean

Leandra krugii (Cogn.) Judd \& Skean

Leandra lacunosa Cogn.

Leandra laevigata (Triana) Cogn.

Leandra lancifolia Cogn.

Leandra lapae D'El Rei Souza \& Baumgratz

Leandra lasiopetala Cogn.

Leandra lasiostachya Cogn.

Leandra lehmanni Cogn.

Leandra lima (Desr.) Judd \& Skean

Leandra limbata Cogn.

Leandra limoides (Urb.) Judd \& Skean
Miconia fontanae (Reginato \& R.Goldenb.) R.Goldenb. Miconia leafoveolata R.Goldenb.

Miconia secunfrancavillana G. Ocampo \& Almeda

Miconia freyreissii (Cogn.) R.Goldenb.

Miconia dolichostachya (Naudin) R.Goldenb.

Miconia glanduliflora R.Goldenb. \& Michelang.

Miconia pubistyla (Wurdack) R.Goldenb.

Miconia gorzulae (Wurdack) Michelang. \& R.Goldenb.

Miconia leagracilis R.Goldenb.

Miconia granatensis (Gleason) G. Ocampo \& Almeda

Miconia secungrandifolia G. Ocampo \& Almeda

Miconia leagrayana R.Goldenb.

Miconia gynoverrucosa (Reginato) R.Goldenb.

Miconia hatschbachii (Brade) R.Goldenb.

Miconia herincquiana (Cogn.) R.Goldenb.

Miconia hermogenesii (Baumgratz \& D'El Rei Souza)

R.Goldenb.

Miconia heteroporata (Reginato) R.Goldenb.

Miconia leahirtaella R.Goldenb.

Miconia horrida (Cogn.) R.Goldenb.

Miconia leahumilis R.Goldenb.

Miconia hybophylla (Urb.) Majure \& Judd

Miconia inaequalis R.Goldenb. \& Michelang.

Miconia inaequipetiolata Majure \& Judd

Miconia intermedia (DC.) G. Ocampo \& Almeda

Miconia ionopogon (Mart.) R.Goldenb.

Miconia itatiaiae (Wawra) R.Goldenb.

Miconia leakleinii R.Goldenb.

Miconia krugiana (Cogn.) Majure \& Judd

Miconia karlkrugii Majure \& Judd

Miconia lacunosa (Cogn.) R.Goldenb.

Miconia ciliolata (Cogn.) R.Goldenb.

Miconia lealancifolia R.Goldenb.

Miconia lapae (D'El Rei Souza \& Baumgratz)

R.Goldenb.

Miconia lasiopetala (Cogn. ) Almeda

Miconia lasiostachya (Cogn.) R.Goldenb.

Miconia friedrichlehmanni R.Goldenb.

Miconia lima (Desr.) M. Gómez

Miconia limbata (Cogn.) R.Goldenb.

Miconia limoides (Urb.) Majure \& Judd 
Leandra lindeniana (Naudin) Cogn.

Leandra loligomorpha (R.Goldenb. \& Reginato)

Reginato

Leandra longibarbis (Schrank \& Mart. ex DC.)

Cogn.

Leandra longicoma Cogn.

Leandra longisepala Wurdack

Leandra luctatoris Wurdack

Leandra lutea Cogn.

Leandra macdanielii Wurdack

Leandra macropora (O. Berg. ex Triana) Cogn.

Leandra magdalenensis Brade

Leandra magnipetala R. Goldenb \& E. Camargo

Leandra maguirei Wurdack

Leandra marigotiana (Urb. \& Ekman) Alain

Leandra markgrafii Brade

Leandra mattosii Baumgratz \& D'El Rei Souza

Leandra melanodesma (Naudin) Cogn .

Leandra melastomoides Raddi

Leandra mexicana (Naudin) Cogn.

Leandra miconiastrum (Naudin) Cogn .

Leandra micrantha Wurdack

Leandra micropetala (Naudin) Cogn.

Leandra microphylla Cogn.

Leandra mollis Cogn.

Leandra mouraei Cogn.

Leandra multiplinervis (Naudin) Cogn.

Leandra multisetosa Cogn.

Leandra nanayensis Wurdack

Leandra navicularis Brade

Leandra neblinensis Wurdack

Leandra neglecta Brade

Leandra nervosa (Naudin) Cogn.

Leandra neurotricha Cogn.

Leandra nianga (DC) Cogn.

Leandra niangaeformis Cogn.

Leandra niederleinii Cogn.

Leandra oblongifolia Cogn.

Leandra oligochaeta Cogn.

Leandra opaca Brade
Miconia lealindeniana Michelang. \& R.Goldenb.

Miconia loligomorpha (R.Goldenb. \& Reginato)

R.Goldenb.

Miconia longibarbis (Schrank \& Mart. ex DC.)

R.Goldenb.

Miconia longicoma (Cogn. ) G. Ocampo \& Almeda

Miconia secunlongisepala G. Ocampo \& Almeda

Miconia luctatoris (Wurdack) R.Goldenb.

Miconia lutea (Cogn.) R.Goldenb.

Miconia secunmacdanielii G. Ocampo \& Almeda

Miconia macropora (Triana) R.Goldenb.

Miconia magdalenensis (Brade) R.Goldenb.

Miconia magnipetala (R.Goldenb \& E. Camargo)

R.Goldenb.

Miconia secunmaguirei G. Ocampo \& Almeda

Miconia marigotiana (Urb. \& Ekm.) Majure \& Judd

Miconia leamarkgrafii R.Goldenb.

Miconia mattosii (Baumgratz \& D'El Rei Souza)

R.Goldenb.

Miconia melanodesma (Naudin) Michelang. \&

R.Goldenb.

Miconia melastomoides (Raddi) R.Goldenb.

Miconia secunmexicana G. Ocampo \& Almeda

Miconia miconiastrum (Naudin) R.Goldenb.

Miconia microflora R.Goldenb. \& Michelang.

Miconia nanopetala R.Goldenb. \& Michelang.

Miconia dusenii (Cogn.) R.Goldenb.

Miconia leamollis R.Goldenb.

Miconia leamourae R.Goldenb.

Miconia oocarpa (A.Gray) R.Goldenb.

Miconia multisetosa (Cogn.) R.Goldenb.

Miconia nanayensis (Wurdack) R.Goldenb. \& Michelang.

Miconia navicularis (Brade) R.Goldenb.

Miconia secunneblinensis G. Ocampo \& Almeda

Miconia neglecta (Brade) R.Goldenb.

Miconia nervosissima Michelang. \& R.Goldenb.

Miconia leaneurotricha R.Goldenb.

Miconia nianga (DC.) R.Goldenb.

Miconia niangaeformis (Cogn.) R.Goldenb.

Miconia niederleinii (Cogn.) R.Goldenb.

Miconia leaoblongifolia R.Goldenb.

Miconia oligochaeta (Cham.) R.Goldenb.

Miconia opaca (Brade) R.Goldenb. 
Leandra organensis Cogn.

Leandra ossaeoides (Naudin) Cogn.

Leandra ovata Cogn.

Leandra paleacea Wurdack

Leandra pallida Cogn.

Leandra papillata Cogn.

Leandra pastazana Wurdack

Leandra paulina DC.

Leandra pectinata Cogn.

Leandra peltata Wurdack

Leandra penduliflora (Naudin) Cogn.

Leandra pennipilis (O. Berg. ex Triana) Cogn.

Leandra phelpsiae Gleason

Leandra pilonensis Wurdack

Leandra pilosissima Cogn.

Leandra planifilamentosa Brade

Leandra polyadena Ule

Leandra polychaeta (Urb. \& Ekm.) Alain

Leandra polychaeta Cogn.

Leandra polystachya (Naudin) Cogn.

Leandra procumbens Ule

Leandra purpurascens (DC.) Cogn.

Leandra purpurea Gleason

Leandra purpureo-villosa Hoehne

Leandra quinquedentata (DC.) Cogn.

Leandra quinquenodis (DC) Cogn.

Leandra raimondiana Markgr.

Leandra ramboi Brade

Leandra refracta Cogn.

Leandra regnellii (Triana) Cogn.

Leandra reitzii Wurdack

Leandra reptans R.Goldenb. \& Reginato

Leandra retropila Cogn.

Leandra reversa (DC.) Cogn.

Leandra rhamnifolia (Naudin) Cogn.

Leandra rhodopogon (DC.) Cogn.

Leandra ribesiaeflora (Cham.) Cogn.

Leandra riedeliana (O. Berg. ex Triana) Cogn.

Leandra rigida Cogn.

Leandra riograndensis (Brade) Wurdack
Miconia leaorganensis R.Goldenb.

Miconia ossaeoides (Naudin) Michelang. \& Tiernan

Miconia leaovata R.Goldenb.

Miconia paleaciramis Michelang. \& R.Goldenb.

Miconia leapallida R.Goldenb.

Miconia papillata (Cogn.) R.Goldenb.

Miconia secunpastazana G. Ocampo \& Almeda

Miconia paulina (DC.) R.Goldenb.

Miconia pectinata (Cogn.) R.Goldenb.

Miconia peltifolia R.Goldenb. \& Michelang.

Miconia dolichodons (Cogn.) R.Goldenb.

Miconia leapennipilis R.Goldenb.

Miconia phelpsiae (Gleason) R.Goldenb. \& Michelang.

Miconia pilonensis (Wurdack) R.Goldenb.

Miconia pilosissima (Cogn.) R.Goldenb.

Miconia planifilamentosa (Brade) R.Goldenb.

Miconia polyadena (Ule) R.Goldenb. \& Michelang.

Miconia pedunculata Majure \& Judd

Miconia leapolychaeta R.Goldenb.

Miconia polystachya (Naudin) R.Goldenb.

Miconia chimantensis (Wurdack) Michelang.

Miconia microstachya (Naudin) R.Goldenb.

Miconia purpuriflora Michelang. \& R.Goldenb.

Miconia purpureovillosa (Hoehne) R.Goldenb.

Miconia quinquedentata (DC.) R.Goldenb.

Miconia quinquenodis (DC.) R.Goldenb.

Miconia raimondiana (Markgr.) R.Goldenb. \&

Michelang.

Miconia learamboi R.Goldenb.

Miconia refracta (Cogn.) R.Goldenb.

Miconia alterninervia (Cogn.) R.Goldenb.

Miconia reitzii (Wurdack) R.Goldenb.

Miconia reptans (R.Goldenb. \& Reginato) R.Goldenb.

Miconia secunretropila G. Ocampo \& Almeda

Miconia reversa (DC.) G. Ocampo \& Almeda

Miconia rhamnifolia (Naudin) G. Ocampo \& Almeda

Miconia rhodopogon (DC.) R.Goldenb.

Miconia ribesiiflora (DC.) R.Goldenb.

Miconia leariedeliana R.Goldenb.

Miconia learigida R.Goldenb.

Miconia riograndensis (Brade) R.Goldenb. 
Leandra rotundifolia J.F.Macbr.

Leandra rubida Cogn.

Leandra rufescens (DC.) Cogn.

Leandra sabiaensis Brade

Leandra saldanhaei Cogn.

Leandra salicina (Ser. ex DC.) Cogn.

Leandra sanguinea Gleason

Leandra santos-limae Brade

Leandra secunda (D.Don) Cogn.

Leandra secundiflora (DC.) Cogn.

Leandra sericea DC.

Leandra simplicicaulis (Naudin) Cogn.

Leandra solenifera Cogn.

Leandra sparsisetulosa Hoehne

Leandra sphaerocarpa Cogn.

Leandra stellulata Cogn.

Leandra steyermarkii Wurdack

Leandra strigilliflora (Naudin) Cogn.

Leandra subobruta Wurdack

Leandra subseriata (Naudin) Cogn.

Leandra subtrinervis Cogn.

Leandra subulata Gleason

Leandra sulfurea (Naudin) Cogn.

Leandra sylvatica Cogn.

Leandra ternata Cogn.

Leandra tetragona Cogn.

Leandra tetraptera Cogn.

Leandra tetraquetra (Cham.) Cogn.

Leandra therezopolitana Cogn.

Leandra thyrsiflora Markgr. ex Pilg.

Leandra tomentosa Cogn.

Leandra triantha R. Goldenb \& E. Camargo

Leandra tristis Cogn.

Leandra truncata Baumgratz \& D'El Rei Souza

Leandra ulaei Cogn.

Leandra uliginosa Brade

Leandra umbellata DC.

Leandra variabilis Raddi

Leandra velutina (Gardn.) Cogn.

Leandra verticillata (DC.) Cogn.
Miconia secunrotundifolia G. Ocampo \& Almeda

Miconia rubida (Cogn.) R.Goldenb.

Miconia asperiuscula (Rich. ex DC.) R.Goldenb.

Miconia sabiaensis (Brade) R.Goldenb.

Miconia leasaldanhae R.Goldenb.

Miconia salicina (Ser. Ex DC.) Mabb.

Miconia secunsanguinea G. Ocampo \& Almeda

Miconia santoslimae (Brade) R.Goldenb.

Miconia neosecunda G. Ocampo \& Almeda

Miconia neosecundiflora G. Ocampo \& Almeda

Miconia raddii R.Goldenb.

Miconia simplicicaulis (Naudin) R.Goldenb.

Miconia solenifera (Cogn. ) G. Ocampo \& Almeda

Miconia sparsisetulosa (Hoehne) R.Goldenb.

Miconia sphaerocarpa (Cogn.) R.Goldenb.

Miconia stellulitricha R.Goldenb. \& Michelang.

Miconia juliansteyermarkii R.Goldenb. \& Michelang.

Miconia strigilliflora (Naudin) R.Goldenb.

Miconia subobruta (Wurdack) R.Goldenb. \& Michelang.

Miconia subseriata (Naudin) R.Goldenb. \& Michelang.

Miconia subtrinervis (Cogn.) R.Goldenb.

Miconia subulata (Gleason) R.Goldenb. \& Michelang.

Miconia sulfurea (Naudin) R.Goldenb.

Miconia leasylvatica R.Goldenb.

Miconia ternata (Cogn.) R.Goldenb.

Miconia leatetragona R.Goldenb.

Miconia leatetraptera R.Goldenb.

Miconia tetraquetra (Cham.) R.Goldenb.

Miconia therezopolitana (Cogn.) R.Goldenb.

Miconia leathyrsiflora R.Goldenb.

Miconia leatomentosa R.Goldenb.

Miconia triantha (R.Goldenb. \& E.Camargo) R.Goldenb.

Miconia leatristis R.Goldenb.

Miconia leatruncata R.Goldenb.

Miconia ulei (Cogn.) R.Goldenb.

Miconia uliginosa (Brade) R.Goldenb.

Miconia leaumbellata R.Goldenb.

Miconia dasytricha (A.Gray) R.Goldenb.

Miconia leavelutina R.Goldenb.

Miconia verticillifolia Michelang. \& R.Goldenb. 
Leandra vesiculosa Cogn.

Leandra viridiflava Brade

Leandra viscosa Cogn.

Leandra warmingiana Cogn.

Leandra xanthocoma (Naudin) Cogn.

Leandra xantholasia (DC.) Cogn.

Leandra xanthopogon (Naudin) Cogn.

Leandra xanthostachya Cogn.

Llewelynia wiliamsii Pittier

Loreya arborescens (Aubl.) DC.

Loreya gracilis S.S.Renner

Loreya huberi Wurdack

Loreya klugii S.S.Renner

Loreya mespiloides Miq.

Loreya mucronata Gleason

Loreya nigricans (Hook. f.) Triana

Loreya ovata O.Berg ex Triana

Loreya riparia S.S.Renner

Loreya spruceana Benth. ex Triana

Loreya strigosa Gleason

Loreya subandina Wurdack

Loreya subrotundifolia (Wurdack) S.S.Renner

Loreya umbellata (Gleason) Wurdack

Loreya wurdackiana S.S.Renner

Maieta guianensis Aubl.

Maieta neblinensis Wurdack

Maieta poeppigii Mart. ex Cogn.

Mecranium acuminatum (DC.) Skean

Mecranium alpestre Urb. \& Ekman

Mecranium amygdalinum (Desr.) C.Wright

Mecranium axillare (Macfadyen) Skean

Mecranium axillare subsp. proctorii Skean

Mecranium axillare subsp. urbanianum (Cogn.)

Skean

Mecranium birimosum (Naudin) Triana

Mecranium crassinerve (Urb.) Skean
Miconia vesiculosa (Cogn.) R.Goldenb.

Miconia viridiflava (Brade) R.Goldenb.

Miconia viscosa (Cogn.) R.Goldenb.

Miconia leawarmingiana R.Goldenb.

Miconia xanthocoma (Naudin) R.Goldenb.

Miconia xantholasia (DC.) R.Goldenb.

Miconia xanthopogon (Naudin) R.Goldenb.

Miconia xanthostachya (Cogn.) R.Goldenb.

Henriettea williamsii (Pittier) Penneys, Michelangeli,

Judd \& Almeda

Bellucia arborescens (Aubl.) Baill.

Bellucia gracilis (Renner) Penneys, Michelangeli, Judd \& Almeda

Bellucia huberi (Wurdack) S.S.Renner

Bellucia klugii (Renner) Penneys, Michelangeli, Judd \&

Almeda

Bellucia mespiloides (Miq.) J.F.Macbr.

Henriettea mucronata (Gleason) S.S.Renner

Bellucia nigricans (Hook. f.) Penneys, Michelangeli, Judd

\& Almeda

Bellucia ovata (O. Berg. ex Triana) Penneys,

Michelangeli, Judd \& Almeda

Bellucia riparia (Renner) Penneys, Michelangeli, Judd \& Almeda

Bellucia spruceana (Benth. ex Triana) J.F.Macbr.

Bellucia strigosa (Gleason) Penneys, Michelangeli, Judd

\& Almeda

Bellucia subandina (Wurdack) Penneys, Michelangeli,

Judd \& Almeda

Bellucia subrotundifolia Wurdack

Bellucia umbellata Gleason

Bellucia wurdackiana (Renner) Penneys, Michelangeli, Judd \& Almeda

Miconia mayeta (D. Don) Michelang.

Miconia alatiflora Michelang.

Miconia alternidomatia Michelang.

Miconia acuminoides Skean, Judd \& Majure

Miconia rufoalpestris Skean, Judd \& Majure

Miconia neoamygdalina Skean, Judd \& Majure

Miconia axillaris (Macfad.) Skean, Judd \& Majure

Miconia Miconia axillare subsp. proctori (Skean) Skean

Miconia Miconia axillaris subsp. urbaniana (Skean)

Skean

Miconia birrimosa (naudin) Skean, Judd \& Majure

Miconia crassinervis (Urb.) Skean, Judd \& Majure 
Mecranium haemanthum Triana ex Cogn.

Mecranium haitiense Urb.

Mecranium integrifolium (Naudin) Triana

Mecranium integrifolium subsp. alainii Skean

Mecranium integrifolium subsp. neibense (Skean)

Skean

Mecranium juddii Skean

Mecranium latifolium (Cogn.) Skean

Mecranium microdictyum Urb. \& Ekman

Mecranium multiflorum (Desr.) Triana

Mecranium obtusifolium Cogn.

Mecranium ovatum Cogn.

Mecranium plicatum Urb.

Mecranium puberulum Cogn.

Mecranium purpurascens (DC.) Triana

Mecranium racemosum (Griseb.) C.Wright

Mecranium revolutum Skean \& Judd

Mecranium septentrionale Skean

Mecranium tricostatum Urb. \& Ekman

Mecranium tuberculatum Urb.

Mecranium virgatum (Sw.) Triana

Miconiastrum lambertianum Bonpl. ex Naudin

Microlepis oleaefolia DC.

Myriaspora egensis DC.

Necramium gigantophyllum Britton

Ossaea acunae

Ossaea amygdaloides (DC.) Triana

Ossaea angustifolia (DC.) Triana

Ossaea araneifera Markgr.

Ossaea asperifolia (Naudin) Triana

Ossaea asplundii Wurdack

Ossaea baracoensis Borhidi \& O. Muñiz

Ossaea boekei Wurdack

Ossaea boliviensis (Cogn.) Gleason

Ossaea brachystemon Urb

Ossaea bracteata Triana

Ossaea brenesii Standley

Ossaea brunescens Urb.
Miconia haemantha (Triana ex Cogn.) Skean, Judd \& Majure

Miconia ayisyena Skean, Judd \& Majure

Miconia antillana Skean, Judd \& Majure

Miconia Miconia antillana subsp. alainii (Skean) Skean, Judd \& Majure

Miconia Miconia antillana subsp. neibensis (Skean)

Skean, Judd \& Majure

Miconia juddii (Skean) Skean, Judd \& Majure

Miconia borinquensis Skean, Judd \& Majure

Miconia microdicta (Urb. \& Ekman) Skean, Judd \&

Majure

Miconia polyflora Skean, Judd \& Majure

Miconia obtusifolia (Cogn.) Skean, Judd \& Majure

Miconia bahorucensis Skean, Judd \& Majure

Miconia plicatifolia Skean, Judd \& Majure

Miconia hirticaulis Skean, Judd \& Majure

Miconia ossaeiformis Naudin

Miconia conica Skean, Judd \& Majure

Miconia revolutifolia Skean, Judd \& Majure

Miconia ocampensis Skean, Judd \& Majure

Miconia tricostata (Urb. \& Ekman) Skean, Judd \&

Majure

Miconia haemanthoides Skean, Judd \& Majure

Miconia virgata (Sw.) Skean, Judd \& Majure

Miconia maestrensis Judd, Bécquer, \& Majure

Pleroma oleifolium (DC.) R. Romero \& Versiane

Bellucia egensis (DC.) Penneys, Michelangeli, Judd \& Almeda

Miconia gigantophylla (Britton) Michelang.

Miconia acunagalei Judd, Bécquer \& Majure

Miconia amygdaloides (DC.) R.Goldenb.

Miconia corcovadensis (Raddi) R.Goldenb.

Miconia araneifera (Markg.) Michelang. \& R.Goldenb.

Miconia asperifolia (Naudin) Majure \& Judd

Miconia alboglandulosa Gamba \& Almeda

Miconia borhidiana Judd, Bécquer \& Majure

Miconia boekei (Wurdack) Gamba \& Almeda

Miconia violascens (Pilg.) Michelang. \& R.Goldenb.

Miconia brachystemon (Urb.) Judd, Bécquer \& Majure

Miconia bractiflora Gamba \& Almeda

Miconia albertobrenesii Gamba \& Almeda

Miconia brunescens ((Urb.)) Bécquer \& Skean 
Ossaea buchtienii Gleason

Ossaea bullifera (Pilg.) Gleason

Ossaea cabraliensis (Wurdack) D'El Rei Souza

Ossaea capillaris (D.Don) Cogn.

Ossaea capitata Urb.

Ossaea cinnamomifolia (Cham. ex Naudin) Triana

Ossaea coarctiflora Wurdack

Ossaea confertiflora (DC.) Triana

Ossaea congestiflora Cogn.

Ossaea coriacea (Naudin) Triana

Ossaea costata Urb.

Ossaea cubana Alain

Ossaea cucullata Gleason

Ossaea cuneata Cogn.

Ossaea echinata (Griseb.) M. Gómez

Ossaea ekmanii Urb.

Ossaea elliptica Alain

Ossaea euphorbioides (Naudin) Triana

Ossaea filisepala Urb.

Ossaea filisepala Urb.

Ossaea fragilis Cogn.

Ossaea glomerata (Naudin) Triana

Ossaea granulata Urb.

Ossaea heterotricha (Griseb.) C. Wright

Ossaea hirsuta (Sw.) Triana

Ossaea hirtella (DC.) Triana

Ossaea hypoglauca (C. Wright ex Griseb.) M.

Gómez

Ossaea incerta Wurdack

Ossaea involucrata (Griseb.) C. Wright

Ossaea lanata (Naudin) C. Wright ex Griseb.

Ossaea lanceolata Urb.

Ossaea laxivenula Wurdack

Ossaea macrophylla (Benth.) Cogniaux

Ossaea marginata (Desr.) Triana

Ossaea mavacana Wurdack

Ossaea meridionalis D'El Rei Souza

Ossaea micrantha (Sw.) Cogn.

Ossaea microphylla (Sw.) C. Wright

Ossaea moaensis Alain

Ossaea muricata (Griseb.) C. Wright
Miconia ottobuchtienii Michelang. \& R.Goldenb.

Miconia myrmecodomatia Michelang.

Miconia cabraliensis (Wurdack) R.Goldenb.

Miconia leptopus (Triana) Michelang. \& R.Goldenb.

Miconia tentaculicapitata Majure \& Judd

Miconia leacinnamomifolia R.Goldenb.

Miconia coarctiflora (Wurdack) G. Ocampo \& Almeda

Miconia rubella (Raddi) R.Goldenb.

Miconia leacongestiflora R.Goldenb.

Miconia leabiscoriacea R.Goldenb.

Miconia costata (Urb.) Judd, Bécquer \& Majure

Miconia cubana (Alain) Majure \& Judd

Miconia cucullata (Gleason) Michelang.

Miconia leacuneata R.Goldenb.

Miconia echinata (Griseb.) Judd, Bécquer \& Majure

Miconia ekmanii (Urb.) Judd, Bécquer \& Majure

Miconia ascenditricha Judd, Bécquer \& Majure

Miconia euphorbioides (Naudin) R.Goldenb.

Miconia filisepala (Urb.) Judd, Bécquer \& Majure

Miconia filisepala (Urb.) Judd, Bécquer \& Majure

Miconia leafragilis R.Goldenb.

Miconia glomeruliflora Judd, Bécquer\&Majure

Miconia granulata (Urb.) Majure \& Judd

Miconia yunquensis Judd, Bécquer \& Majure

Miconia hirsuta (Sw.) Judd, Bécquer \& Majure

Miconia hirtellicaulis Ionta \& Judd

Miconia hypoglauca (C. Wright ex Griseb.) Judd,

Bécquer\& Majure

Miconia incerta (Wurdack) Gamba \& Almeda

Miconia grandibracteata Judd, Bécquer \& Majure

Miconia lanatifolia Judd, Bécquer \& Majure

Miconia echinocarpa Judd, Bécquer \& Majure

Miconia laxivenula (Wurdack) Gamba \& Almeda

Miconia solearis (Naudin) Gamba \& Almeda

Miconia leamarginata R.Goldenb.

Miconia mavacana (Wurdack) Michelang.

Miconia meridionalis (D'El Rei Souza) R.Goldenb.

Miconia rubescens (Triana) Gamba \& Almeda

Miconia pratensis Judd, Bécquer \& Majure

Miconia lutgardae Becquer \& Michelang.

Miconia argentimuricata Majure \& Judd 
Ossaea navasensis Britton \& P.Wilson

Ossaea neurotricha C.Wright

Ossaea nipensis Britton \& P.Wilson

Ossaea norlindii Urb.

Ossaea ottoschmidtii Urb.

Ossaea ovatifolia Urb.

Ossaea palenquensis Wurdack

Ossaea parvifolia Alain

Ossaea petiolaris (Naudin) Triana

Ossaea pilifera Urb.

Ossaea pinetorum Alain

Ossaea pseudopinetorum Borhidi \& O. Muñiz

Ossaea pulchra Alain

Ossaea pulverulenta Urb.

Ossaea quadrisulca (Naudin) Wurdack

Ossaea resinosa Gleason

Ossaea retropila (DC.) Triana

Ossaea robusta (Triana) Cogniaux

Ossaea rubrinervis Urb. \& Ekman

Ossaea rufescens (Griseb.) Wright

Ossaea rufibarbis Triana

Ossaea sanguinea Cogn.

Ossaea secundiflora Cogn.

Ossaea sessilifolia (Triana) Wurdack

Ossaea shaferi Britton \& Wilson

Ossaea sparrei Wurdack

Ossaea spicata Gleason

Ossaea sulbahiensis D'El Rei Souza

Ossaea suprabasalis R.Goldenb. \& Reginato

Ossaea trianaei Cogn.

Ossaea turquinensis Urb

Ossaea vazquezii Borhidi \& O.Muniz

Ossaea verrucosa (Griseb.) M. Gómez

Ossaea warmingiana Cogn.

Ossaea wilsonii Alain

Ossaea wrightii Triana

Pachyanthus angustifolius Griseb.

Pachyanthus blancheanus (Urb.) Urb. \& Ekman
Miconia navasenssis (Briton \& P Wilson) Ionta \& Becquer

Miconia capillinervis Ionta \& Judd

Miconia nipensis (Briton \& P Wilson) Ionta \& Becquer

Miconia norlindii (Urb.) Majure \& Judd

Miconia ottoschmidtii (Urb.) Majure \& Judd

Miconia ovatifolia (Urb.) Judd, Bécquer \& Majure

Miconia palenquensis (Wurdack) Gamba \& Almeda

Miconia nanophylla Judd, Bécquer \& Majure

Miconia secunpetiolaris G. Ocampo \& Almeda

Miconia pilifera (Urb.) Ionta \& Becquer

Miconia pseudopinetorum (Alain) Judd, Bécquer \&

Majure

Miconia pseudopinetorum (Alain) Judd, Bécquer \&

Majure

Miconia vazquezii (Borhidi \& Muniz) Ionta \& Becquer

Miconia pulverata Judd, Bécquer \& Majure

Miconia quadrisulca (Naudin) G. Ocampo \& Almeda

Miconia resinosa (Gleason) Michelang.

Miconia retropila (Triana) G. Ocampo \& Almeda

Miconia variabilis Gamba \& Almeda

Miconia rufinervis Judd

Miconia ruficaulis Ionta \& Becquer

Miconia rufibarbis (Triana) Gamba \& Almeda

Miconia leasanguinea R.Goldenb.

Miconia bisecundiflora G. Ocampo \& Almeda

Miconia sessilis Gamba \& Almeda

Miconia jashaferi Majure \& Judd

Miconia bensparrei Gamba \& Almeda

Miconia spicata (Gleason) Gamba \& Almeda

Miconia sulbahiensis (D'El Rei Souza) R.Goldenb.

Miconia suprabasalis (R.Goldenb. \& Reginato)

R.Goldenb.

Miconia trianana Judd \& Becquer

Miconia norlindii (Urb.) Majure \& Judd

Miconia vazquezii (Borhidi \& O.Muniz) Ionta \& Becquer

Miconia costata (Urb.) Judd, Bécquer \& Majure

Miconia leabiswarmingiana R.Goldenb.

Miconia scaberrima Judd, Bécquer \& Majure

Miconia munizii (Borhidi) Ionta \& Becquer

Miconia secundo-angustifolia (Griseb.) M. Gomez

Miconia blancheana Urb. 
Pachyanthus clementis P.Wilson

Pachyanthus cubensis A.Rich.

Pachyanthus discolor Norlind ex Urb.

Pachyanthus longifolius Jenn.

Pachyanthus lundellianus (L.O.Williams) Judd \&

Skean

Pachyanthus mantuensis Britton \& P.Wilson

Pachyanthus mayarensis Urb.

Pachyanthus moaensis Borhidi

Pachyanthus monocephalus (Urb.) Borhidi

Pachyanthus monopleurus Urb.

Pachyanthus neglectus Borhidi

Pachyanthus oleifolius Griseb.

Pachyanthus pedicellatus Urb.

Pachyanthus poiretii Griseb.

Pachyanthus reticulatus Britton \& P.Wilson

Pachyanthus tetramerus Urb. \& Ekman

Pachyanthus wrightii Griseb.

Pleiochiton amorimii Reginato \& R.Goldenb.

Pleiochiton blepharodes (DC.) Reginato, R.Goldenb.

\& Baumgratz; comb.

Pleiochiton crassifolium Naudin ex A.Gray

Pleiochiton ebracteatum Triana

Pleiochiton glaziovianum Cogn.

Pleiochiton longipetiolatum Brade

Pleiochiton magdalenense Brade

Pleiochiton micranthum Cogn.

Pleiochiton parasiticum (O'Berg ex Triana) Reginato,

R.Goldenb. \& Baumgratz

Pleiochiton parvifolium Cogn.

Pleiochiton roseum Cogn.

Pleiochiton setulosum Cogn.

Purpurella cleistoflora Ule

Sagraea abbottii (Urb.) Alain

Sagraea barahonensis (Urb. \& Ekman) Alain

Sagraea capillaris (Sw.) DC.

Sagraea cinerea (Cogn.) Alain

Sagraea curvipila (Urb. \& Ekman) Alain

Sagraea ellipsoidea (Urb. \& Ekman) Alain

Sagraea fuertesii (Cogn.) Alain

Sagraea gracilis (Alain) Alain
Miconia clementii (P.Wilson) Becquer

Miconia pachyantha Becquer

Miconia discolorata Becquer

Miconia brachyloba (Urb.) Becquer

Miconia lundelliana L.O.Williams

Miconia mantuensis (Britton \& P.Wilson) Becquer

Miconia mayarensis (Urb.) Becquer \& Michelang.

Miconia yamanigueyensis Becquer \& Michelang.

Miconia monocephala Urb.

Miconia monopleura (Urb.) Becquer \& Majure

Miconia omissa Becquer \& Michelang.

Miconia oleifolia (Griseb.) M. Gomez

Miconia pseudopedicellata Judd \& Becquer

Miconia poiretii (Griseb.) M. Gomez

Miconia lindmanii (Urb.) Becquer

Miconia tetramera (Urb. \& Ekman) Becquer

Miconia wrightii (Griseb.) Triana

Miconia amorimii (Reginato \& R. Goldenb) R.Goldenb.

Miconia blepharodes (DC.) R.Goldenb.

Miconia pleiocrassifolia R.Goldenb.

Miconia ebracteata (Triana) R.Goldenb.

Miconia pleioglazioviana R.Goldenb.

Miconia longipetiolata (Brade) R.Goldenb.

Miconia pleiomagdalenensis R.Goldenb.

Miconia pleiomicrantha R.Goldenb.

Miconia parasitica (O.Berg ex Triana) R.Goldenb.

Miconia pleioparvifolia R.Goldenb.

Miconia pleiorosea R.Goldenb.

Miconia pleiosetulosa R.Goldenb.

Pleroma cleistoflorum (Ule) P.J.F.Guim., Oliveira da

Silva \& Michelang.

Miconia abbottii (Urb.) Ionta \& Judd

Miconia barahonensis (Urb. \& Ekman) Ionta \& Judd

Miconia capillaris (Sw.) Ionta, Judd \& Skean

Miconia cinereiformis Ionta, Judd \& Skean

Miconia curvipila (Urb. \& Ekman) Ionta, Judd \& Skean

Miconia ellipsoidea (Urb. \& Ekman) Ionta, Judd \& Skean

Miconia miguelfuertesii Ionta \& Judd

Miconia gracilifolia Ionta \& Judd 
Sagraea lanceifolia (Urb.) Alain

Sagraea oligantha (Urb.) Alain

Sagraea penninervis (Griseb.) Triana

Sagraea polychaete (Urb. \& Ekman) Alain

Sagraea pusilliflora (Cogn.) Alain

Sagraea scabrosa (L.) Naudin

Sagraea setulosa (Urb.) Alain

Sagraea woodsii (Judd \& Skean) Alain

Salpinga margaritacea (Naudin) Triana

Svitramia hatschbachii Wurdack

Svitramia integerrima R.Romero \& A.B.Martins

Svitramia minor R.Romero \& A.B.Martins

Svitramia petiolata R.Romero \& A.B.Martins

Svitramia pulchra Cham.

Svitramia wurdackiana R.Romero \& A.B.Martins

Tetrazygia albicans Triana

Tetrazygia angustiflora (Benth.) Griseb.

Tetrazygia angustifolia (Sw.) DC.

Tetrazygia aurea R.A.Howard \& W.R.Briggs

Tetrazygia barbata Borhidi

Tetrazygia bicolor (Mill.) Cogn.

Tetrazygia bicolor var. patenti-setosa Borhidi

Tetrazygia biflora (Cogn.) Urb.

Tetrazygia brachycentra (Griseb.) C. Wright

Tetrazygia cordata Alain

Tetrazygia coriacea Urb.

Tetrazygia cristalensis Borhidi

Tetrazygia decorticans Becquer

Tetrazygia discolor (L.) DC.

Tetrazygia ekmanii Urb.

Tetrazygia elaeagnoides (Sw.) DC

Tetrazygia elegans Urb.

Tetrazygia fadyenii Hook.

Tetrazygia hispida (Sw.) Macfad.

Tetrazygia impressa Urb

Tetrazygia lanceolata Urb.,

Tetrazygia laxiflora Naudin

Tetrazygia longicollis Urb. \& Cogn.
Miconia lanceifolia (Urb.) Ionta, Judd \& Skean

Miconia spanantha Judd \& Majure

Miconia penninervis (Griseb.) M. Gomez

Miconia polychaete (Urb. \& Ekman) Ionta, Judd \& Skean

Miconia portoricensis (Alain) Ionta, Judd \& Skean

Miconia scabrosa (L.) Ionta, Judd \& Skean

Miconia rubrisetulosa Ionta, Judd \& Skean

Miconia woodsii (Judd \& Skean) Ionta, Judd \& Skean

Bertolonia margaritacea Naudin

Pleroma gertii P.J.F. Guim. \& Michelang.

Pleroma integerrima (R.Romero \& A.B.Martins) P.J.F.

Guim. \& Michelang.

Pleroma minus (R.Romero \& A.B.Martins) P.J.F. Guim.

\& Michelang.

Pleroma petiolatum (R.Romero \& A.B.Martins) P.J.F.

Guim. \& Michelang.

Pleroma bandeirae P.J.F. Guim. \& Michelang.

Pleroma wurdackianum (R.Romero \& A.B.Martins)

P.J.F. Guim. \& Michelang.

Miconia proctorii Judd

Miconia angustiflora (Benth.) Naudin

Miconia angustifolia (Sw.) Griseb.

Miconia aurifolia Becquer \& Judd

Miconia barbata (Borhidi) Judd, Bécquer, \& Majure

Miconia bicolor (Mill.) Triana

Miconia bicolor var. patenti-setosa (Borhidi) Judd,

Bécquer, \& Majure

Miconia biflora (Cogn.) Judd

Miconia brachycentra (Griseb.) M. Gomez

Miconia cordilamina Becquer \& Judd

Miconia cajalbanensis Judd, Bécquer, \& Majure

Miconia cristalensis (Borhidi) Judd, Bécquer, \& Majure

Miconia decorticans (Becquer) Becquer \& Majure

Miconia christophoriana (Ham.) DC.

Miconia tetrazygioidea Becquer \& Judd

Miconia elaeagnoides (Sw.) Griseb.

Miconia elegantissima Becquer \& Judd

Miconia fadyenii (Hook.) Judd \& Skean

Miconia glandulosa (Sw.) Naudin

Miconia impressa (Urb.) Judd, Bécquer, \& Majure

Miconia guajaibonensis Judd, Bécquer, \& Majure

Miconia rangeliana C.Wright ex Griseb.

Miconia longicollis (Urb. \& Ekman) Judd \& Becquer 
Tetrazygia paralongicollis Judd, Ionta, Clase \& Skean

Tetrazygia urbani Cogn.

Tibouchina ackermannii Cogn.

Tibouchina ademarii P.J.F.Guim., R. Romero \& Leoni

Tibouchina adenophora Cogn.

Tibouchina alata Cogn.

Tibouchina almedae Todzia

Tibouchina alpestris Cogn.

Tibouchina amoena Herzog

Tibouchina anderssonii Wurdack

Tibouchina andreana Cogn.

Tibouchina angustifolia Cogn.

Tibouchina apparicioi Brade

Tibouchina araneicalyx de Santiago

Tibouchina arborea Cogn.

Tibouchina arenaria Cogn.

Tibouchina aristeguietae Wurdack

Tibouchina arthrostemmoides Cogn.

Tibouchina asperifolia Cogn.

Tibouchina asperior (Cham.) Cogn.

Tibouchina aurea Cogn.

Tibouchina australis Cogn.

Tibouchina axillaris Cogn.

Tibouchina bahiensis Wurdack

Tibouchina barnebyana Wurdack

Tibouchina benthamiana Cogn.

Tibouchina bergiana Cogn.

Tibouchina blanchetiana Cogn.

Tibouchina boraceiensis Brade

Tibouchina boudetii P.J.F.Guim. \& R.Goldenb.

Tibouchina bracteolata J.G. Freitas, A.K.A. Santos \&

R.P. Oliveira

Tibouchina bradeana Renner
Miconia paralongicollis (Judd et al.) Judd \& Becquer

Miconia urbanii (Cogn.) Judd

Pleroma ackermannii (Cogn.) P. J. F. Guim. \&

Michelang.

Pleroma ademarii (P.J.F.Guim., R.Romero \& Leoni) P. J.

F. Guim. \& Michelang.

Chaetogastra adenophora (Cogn.) P.J.F. Guim. \&

Michelang.

Pleroma alatum (Cogn.) P. J. F. Guim. \& Michelang.

Chaetogastra almedae (Todzia) P.J.F. Guim. \&

Michelang.

Chaetogastra montana P.J.F. Guim. \& Michelang.

Pleroma amoenum (Herzog) P. J. F. Guim. \& Michelang.

Chaetogastra anderssonii (Wurdack) P.J.F. Guim. \&

Michelang.

Chaetogastra andreana (Cogn.) P.J.F. Guim. \&

Michelang.

Pleroma angustifolium (Naud.) Triana

Pleroma apparicioi (Brade) P. J. F. Guim. \& Michelang. Chaetogastra araneicalyx (de Santiago) P.J.F. Guim. \&

Michelang.

Pleroma arboreum Gardner

Pleroma arenarium (Cogn.) P. J. F. Guim. \& Michelang. Andesanthus aristeguietae (Wurdack) P.J.F.Guim. \& Michelang.

Chaetogastra arthrostemmoides (Cogn.) P.J.F. Guim. \&

Michelang.

Chaetogastra asperifolia (Cogn.) P.J.F. Guim. \&

Michelang.

Pleroma asperius (Cham.) Triana

Chaetogastra aurea (Cogn.) P.J.F. Guim. \& Michelang.

Pleroma australe Triana

Pleroma axillare (Cogn.) P. J. F. Guim. \& Michelang.

Pleroma bahiense (Wurdack) P. J. F. Guim. \&

Michelang.

Pleroma barnebyanum (Wurdack) P. J. F. Guim. \&

Michelang.

Pleroma benthamianum Gardner

Pleroma bergianum (Cogn.) P. J. F. Guim. \& Michelang. Pleroma blanchetianum (Cogn.) P. J. F. Guim. \&

Michelang.

Pleroma boraceiensis (Brade) P. J. F. Guim. \&

Michelang.

Pleroma boudetii (P.J.F.Guim. \& R.Goldenb.) P. J. F.

Guim. \& Michelang.

Pleroma bracteolatum (J. G. Freitas, A. K. A.Santos \& R.

$\mathrm{P}$.

Pleroma edmundoi (Brade \& Markgraf) P.J.F. Guim. \&

Michelang. 
Tibouchina breedlovei Wurdack

Tibouchina brittoniana Cogn.

Tibouchina campii Wurdack

Tibouchina candolleana Cogn.

Tibouchina canescens Cogn.

Tibouchina capitata (Naudin) Cogn.

Tibouchina cardinalis Cogn.

Tibouchina carvalhoi Wurdack

Tibouchina castellensis Brade

Tibouchina cerastifolia Cogn.

Tibouchina chamaecistus Cogn.

Tibouchina chamissoana Cogn.

Tibouchina chiapensis Wurdack

Tibouchina chironioides (Triana) Cogn.

Tibouchina ciliaris (Vent.) Cogn.

Tibouchina cinerea Cogn.

Tibouchina cisplatensis Cogn.

Tibouchina cistoides (Griseb.) Cogn.

Tibouchina citrina (Naudin) Cogn.

Tibouchina claussenii (Naudin) Cogn.

Tibouchina clavata (Pers.) Wurdack

Tibouchina clidemioides Cogn.

Tibouchina clinopodifolia (DC.) Cogn.

Tibouchina collina Cogn.

Tibouchina comosa J.G. Freitas, A.K.A. Santos \&

R.P. Oliveira

Tibouchina confertiflora (Naudin) Cogn.

Tibouchina congestiflora Todzia

Tibouchina connata Todzia

Tibouchina cordifolia Cogn.

Tibouchina cornuta Gleason

Tibouchina crassiramis Cogn.

Tibouchina cristata Brade

Tibouchina cryptadena Gleason
Heterocentron breedlovei (Wurdack) P.J.F. Guim. \& Michelang.

Chaetogastra brittoniana (Cogn. ex Rusby) P.J.F. Guim.

\& Michelang.

Chaetogastra campii (Wurdack) P.J.F. Guim. \&

Michelang.

Pleroma candolleanum (Mart. ex. DC.) Triana

Pleroma canescens (D.Don) P. J. F. Guim. \& Michelang. Chaetogastra capitata (Naudin) P.J.F. Guim. \& Michelang.

Pleroma cardinale (Bonpl.) Triana

Pleroma carvalhoi (Wurdack) P. J. F. Guim. \&

Michelang.

Pleroma castellense (Brade) P. J. F. Guim. \& Michelang. Chaetogastra cerastifolia (Naudin) P.J.F. Guim. \&

Michelang.

Chaetogastra chamaecistus Sieber ex Grisebach

Pleroma molle (Cham.) Triana

Heterocentron chiapense (Wurdack) P.J.F. Guim. \&

Michelang.

Chaetogastra chironioides Grisebach

Chaetogastra ciliaris DC.

Pleroma cinereum (Cogn.) P. J. F. Guim. \& Michelang. Chaetogastra cisplatensis (Cogn.) P.J.F. Guim. \& Michelang.

Chaetogastra cistoides Griseb.

Chaetogastra citrina (Naudin) P.J.F. Guim. \& Michelang.

Pleroma claussenii (Naud.) Triana

Pleroma clavatum (Persoon) P. J. F. Guim. \& Michelang.

Pleroma clidemioides Berg. ex Trianna

Chaetogastra clinopodifolia DC.

Pleroma collinum (Naudin) Triana

Pleroma comosum (J. G. Freitas, A. K. A.Santos \& R. P. Chaetogastra confertiflora (Naudin) P.J.F. Guim. \&

Michelang.

Chaetogastra congestiflora (Todzia) P.J.F. Guim. \&

Michelang.

Chaetogastra connata (Gleason ex Todzia) P.J.F. Guim.

\& Michelang.

Pleroma cordifolium (Cogn.) P. J. F. Guim. \&

Michelang.

Chaetogastra cornuta (Gleason) P.J.F. Guim. \&

Michelang.

Pleroma crassirame (Cogn.) P. J. F. Guim. \& Michelang.

Pleroma cristatum (Brade) P. J. F. Guim. \& Michelang. Pleroma cryptadenum (Gleason) P. J. F. Guim. \& Michelang. 
Tibouchina debilis (Cham.) Cogn.

Tibouchina decemcostata Cogn.

Tibouchina decora Gleason

Tibouchina dendroides Cogn.

Tibouchina dimorphophylla Gleason

Tibouchina discolor Brade

Tibouchina divaricata Cogn.

Tibouchina dubia Cogn.

Tibouchina durangensis Standl.

Tibouchina dusenii Cogn.

Tibouchina echinata (Ruiz \& Pav.) Cogn.

Tibouchina eichleri Cogn.

Tibouchina elegans (Gardner) Cogn.

Tibouchina elegantula Todzia \& Almeda

Tibouchina erioclada (Triana) Cogn.

Tibouchina estrellensis (Raddi) Cogn.

Tibouchina excoriata Cogn.

Tibouchina ferrariana Cogn.

Tibouchina fissinervia Cogn.

Tibouchina floribunda Cogn

Tibouchina formosa Cogn.

Tibouchina fothergillae (DC.) Cogn.

Tibouchina foveolata Cogn.

Tibouchina francavillana Cogn.

Tibouchina frigidula (DC.) Cogn.

Tibouchina fulvipilis Cogn.

Tibouchina galeottiana (Naudin) Cogn.

Tibouchina gardneri (Naudin) Cogn.

Tibouchina gardneriana Cogn.

Tibouchina gaudichaudiana Baill.

Tibouchina gayana (Naudin) Cogn.

Tibouchina geitneriana (Schltdl.) Cogn.

Tibouchina gleasoniana Wurdack
Chaetogastra debilis Cham.

Pleroma decemcostatum (Cogn.) P. J. F. Guim. \&

Michelang.

Chaetogastra decora (Gleason) P.J.F. Guim. \&

Michelang.

Pleroma dendroides (Naud.) Triana

Chaetogastra dimorphylla (Gleason) P.J.F. Guim. \&

Michelang.

Pleroma discolor (Brade) P. J. F. Guim. \& Michelang. Pleroma divaricatum (Cogn.) P. J. F. Guim. \&

Michelang.

Pleroma dubium (Cham) P. J. F. Guim. \& Michelang. Chaetogastra durangensis (Standl.) P.J.F. Guim. \& Michelang.

Pleroma dusenii (Cogn.) P. J. F. Guim. \& Michelang.

Chaetogastra echinata (Ruiz et Pav.) DC.

Pleroma eichleri (Cogn.) P. J. F. Guim. \& Michelang.

Pleroma elegans Gardner

Andesanthus elegantulus (Todzia \& Almeda)

P.J.F.Guim.\& Michelang.

Chaetogastra erioclada (Triana) P.J.F. Guim. \&

Michelang.

Pleroma estrellense (Raddi) P.J.F.Guim. \& Michelang. Chaetogastra excoriata (Cogn.) P.J.F. Guim. \&

Michelang.

Chaetogastra ferrariana (Cogn.) P.J.F. Guim. \&

Michelang.

Pleroma fissinervium (Schrank \& Mart. ex DC.) Gardner Pleroma floribundum (Cogn.) P. J. F. Guim. \& Michelang.

Pleroma formosum (Cogn.) P. J. F. Guim. \& Michelang. Pleroma fothergillae (Schrank et Mat. ex DC.) Triana

Pleroma foveolatum (Naud.) Triana

Pleroma francavillanum (Cogn.) P. J. F. Guim. \& Michelang.

Pleroma frigidulum (Schrank et Mart. ex DC.) Triana Chaetogastra fulvipilis (Cogn.) P.J.F. Guim. \& Michelang.

Chaetogastra galeottiana (Naudin) P.J.F. Guim. \& Michelang.

Pleroma gardneri (Naud.) P. J. F. Guim. \& Michelang.

Pleroma echinatum Gardner

Pleroma gaudichaudianum (DC.) A. Gray

Chaetogastra gayana (Naudin) P.J.F. Guim. \&

Michelang.

Chaetogastra geitneriana Schltdl.

Andesanthus gleasonianus (Wurdack) P.J.F.Guim. \& Michelang. 
Tibouchina goldenbergii F.S. Mey., P.J.F.Guim. \& Kozera

Tibouchina gracilis (Bonpl.) Cogn.

Tibouchina granulosa (Desr.) Cogn.

Tibouchina grossa (L. f.) Cogn.

Tibouchina hassleri Cogn.

Tibouchina hatschbachii Wurdack

Tibouchina herbacea (DC.) Cogn.

Tibouchina herincquiana Cogn.

Tibouchina herzogii Cogn.

Tibouchina heteromalla (D. Don) Cogn.

Tibouchina hieracioides Cogn.

Tibouchina hintonii Gleason ex Todzia

Tibouchina hirsutissima Cogn.

Tibouchina hospita Cogn.

Tibouchina hutchisonii Wurdack

Tibouchina incarum Gleason

Tibouchina inopinata Wurdack

Tibouchina itatiaiae Cogn.

Tibouchina kingii Wurdack

Tibouchina kleinii Wurdack

Tibouchina kuhlmannii Brade

Tibouchina kunhardtii Gleason

Tibouchina laevicaulis Wurdack

Tibouchina laevis Cogn.

Tibouchina lanceolata Cogn.

Tibouchina lancifolia Wurdack

Tibouchina langsdorffiana Baill.

Tibouchina latibracteolata P.G.Wilson

Tibouchina latifolia (Naudin) Cogn.

Tibouchina laxa (Desr.) Cogn.

Tibouchina lepidota (Bonpl.) Baill.

Tibouchina lepidota var. congestiflora Bonpl.

Tibouchina lhotzkyana Cogn.
Pleroma goldenbergii (F.S. Mey., P.J.F. Guim. \& Kozera) P. J. F. Guim. \& Michelang.

Chaetogastra gracilis DC.

Pleroma granulosum (Desr.) D. Don

Chaetogastra grossa (L. f.) P.J.F. Guim. \& Michelang.

Chaetogastra hassleri (Cogn.) P.J.F. Guim. \& Michelang. Pleroma hatschbachii (Wurdack) P. J. F. Guim. \& Michelang.

Chaetogastra herbacea (DC.) P.J.F. Guim. \& Michelang. Chaetogastra herincquiana (Cogn.) P.J.F. Guim. \& Michelang.

Chaetogastra herzogii (Cogn.) P.J.F. Guim. \& Michelang.

Pleroma heteromallum (D.Don) D.Don

Chaetogastra hieracioides DC.

Chaetogastra hintonii Gleason ex P.J.F. Guim. \&

Michelang.

Pleroma hirsutissimum (Cogn.) P. J. F. Guim. \&

Michelang.

Pleroma hospitum (Schrank et Mart. ex DC.) Triana

Chaetogastra hutchisonii (Wurdack) P.J.F. Guim. \&

Michelang.

Chaetogastra incarum (Gleason) P.J.F. Guim. \&

Michelang.

Andesanthus inopinatus (Wurdack) P.J.F.Guim. \&

Michelang.

Pleroma itatiaiae (Wawra) P. J. F. Guim. \& Michelang.

Chaetogastra kingii (Wurdack) P.J.F. Guim. \&

Michelang.

Pleroma kleinii (Wurdack) P. J. F. Guim. \& Michelang.

Pleroma kuhlmannii (Brade) P. J. F. Guim. \& Michelang. Chaetogastra kunhardtii (Gleason) P.J.F. Guim. \&

Michelang.

Pleroma laevicaule (Cogn. ex Wurdack) P. J. F. Guim. \& Michelang.

Chaetogastra laevis (Cogn.) P.J.F. Guim. \& Michelang.

Chaetogastra pauciflora P.J.F. Guim. \& Michelang.

Chaetogastra lancifolia (Wurdack) P.J.F. Guim. \&

Michelang.

Pleroma langsdorffianum (Bonpl.) Triana

Chaetogastra latibracteolata (P.G.Wilson) P.J.F. Guim. \&

Michelang.

Chaetogastra latifolia (Naudin) P.J.F. Guim. \&

Michelang.

Chaetogastra laxa (Desr.) P.J.F. Guim. \& Michelang.

Andesanthus lepidotus (Bonpl.) P.J.F.Guim. \&

Michelang.

Andesanthus lepidotus var. congestiflorus (Bonpl.)

P.J.F.Guim. \& Michelang.

Pleroma lhotzkyana (C. Presl.) Triana 
Tibouchina lilacina Cogn.

Tibouchina limae Brade

Tibouchina lithophila Wurdack

Tibouchina longifolia (Vahl) Baill.

Tibouchina longipilosa Cogn.

Tibouchina longisepala Cogn.

Tibouchina longistyla (Cogn.) S.S. Renner

Tibouchina luetzelburgii Markgr.

Tibouchina lutzii Brade

Tibouchina macrochiton Cogn.

Tibouchina macvaughii Todzia

Tibouchina manicata Cogn.

Tibouchina mariae Wurdack

Tibouchina martialis (Cham.) Cogn.

Tibouchina martiusiana (DC.) Cogn.

Tibouchina marumbiensis Wurdack

Tibouchina maximiliana Baill.

Tibouchina melanocalyx R.Romero P.J.F.Guim. \&

Leoni

Tibouchina mello-barretoi Brade

Tibouchina membranifolia Cogn.

Tibouchina microphylla Cogn.

Tibouchina minor Cogn.

Tibouchina minutiflora Cogn.

Tibouchina mollis (Bonpl.) Cogn.

Tibouchina monticola (Naudin) Cogn.

Tibouchina mosenii Cogn.

Tibouchina mouraei Cogn.

Tibouchina mutabilis (Vell.) Cogn.

Tibouchina nanifolia Todzia

Tibouchina narinoensis Wurdack

Tibouchina naudiniana (Decne.) Cogn.

Tibouchina nitida (Graham) Cogn.
Pleroma lilacinum (Cogn.) P. J. F. Guim. \& Michelang.

Pleroma limae (Brade) P. J. F. Guim. \& Michelang. Pleroma lithophilum (Wurdack) P. J. F. Guim. \& Michelang.

Chaetogastra longifolia (Vahl.) DC.

Chaetogastra longipilosa (Cogn.) P.J.F. Guim. \&

Michelang.

Chaetogastra longisepala (Cogn.) P.J.F. Guim. \&

Michelang.

Chaetogastra longistyla (Cogn.) P.J.F. Guim. \&

Michelang.

Pleroma luetzelburgii (Markgr.) P. J. F. Guim. \&

Michelang.

Pleroma lutzii (Brade) P. J. F. Guim. \& Michelang.

Pleroma macrochiton (Mart. ex DC.) Triana

Chaetogastra macvaughii (Todzia) P.J.F. Guim. \&

Michelang.

Pleroma manicatum (Cogn.) P. J. F. Guim. \& Michelang. Chaetogastra mariae (Wurdack) P.J.F. Guim. \& Michelang.

Pleroma martiale (Cham.) Triana

Pleroma martiusianum (DC.) P. J. F. Guim. \&

Michelang.

Pleroma marumbiense (Wurdack) P. J. F. Guim. \&

Michelang.

Pleroma maximilianum (DC.) Triana

Pleroma melanocalyx (R.Romero, P.J.F.Guim. \& Leoni)

P. J. F. Guim. \& Michelang.

Pleroma mellobarretoi (Brade) P. J. F. Guim. \&

Michelang.

Chaetogastra membranifolia (Cogn. \& Rusby) P.J.F.

Guim. \& Michelang.

Pleroma microphyllum (Cogn. ex Schwacke) P. J. F.

Guim. \& Michelang.

Chaetogastra minor (Cogn.) P.J.F. Guim. \& Michelang. Pleroma minutiflorum (Cogn.) P. J. F. Guim. \& Michelang.

Chaetogastra mollis (Bonpl.) DC.

Chaetogastra monticola (Naudin) P.J.F. Guim. \&

Michelang.

Pleroma mosenii (Cogn.) P. J. F. Guim. \& Michelang.

Pleroma mourae (Cogn.) P. J. F. Guim. \& Michelang.

Pleroma mutabile (Vell.) Triana

Chaetogastra nanifolia (Todzia) P.J.F. Guim. \&

Michelang.

Andesanthus narinoensis (Wurdack) P.J.F.Guim. \&

Michelang.

Chaetogastra naudiniana Decne.

Chaetogastra nitida (Graham) P.J.F. Guim. \& Michelang. 
Tibouchina noblickii Wurdack

Tibouchina nodosa Wurdack

Tibouchina obtusifolia Cogn.

Tibouchina ochypetala (Ruiz \& Pav.) Baill.

Tibouchina oligantha Gleason

Tibouchina orbignyana (Naudin) Cogn.

Tibouchina oreophila Wurdack

Tibouchina ornata (Sw.) Baill.

Tibouchina oroensis Gleason

Tibouchina paleacea (Triana) Cogn.

Tibouchina pallida Cogn.

Tibouchina panicularis (Naudin) Britton ex Cogn.

Tibouchina paratropica (Griseb.) Cogn.

Tibouchina parviflora Cogn.

Tibouchina patens Todzia

Tibouchina pauloalvinii Vinha

Tibouchina pendula Cogn.

Tibouchina pentamera (Ule) J.F. Macbr.

Tibouchina pereirae Brade \& Markgr.

Tibouchina pilosa Cogn.

Tibouchina pleromoides J.F. Macbr.

Tibouchina pulcherrima Gleason

Tibouchina purpurascens Cogn.

Tibouchina purpusii Brandegee

Tibouchina quartzophila Brade

Tibouchina raddiana (DC.) Cogn.

Tibouchina radula Markgr.

Tibouchina ramboi Brade

Tibouchina rediviva (Triana) Cogn.

Tibouchina regeliana Cogn.

Tibouchina regnellii Cogn.

Tibouchina reitzii Brade
Pleroma noblickii (Wurdack) P. J. F. Guim. \&

Michelang.

Pleroma nodosum (Wurdack ex Cogn.) P. J. F. Guim. \&

Michelang.

Chaetogastra obtusifolia (Cogn.) P.J.F. Guim. \&

Michelang.

Pleroma ochypetala (Ruiz et Pavon) D. Don

Chaetogastra oligantha (Gleason) P.J.F. Guim. \&

Michelang.

Chaetogastra orbignyana (Naudin) P.J.F. Guim. \&

Michelang.

Pleroma oreophilum (Wurdack) P. J. F. Guim. \&

Michelang.

Chaetogastra inconstans

Chaetogastra oroensis (Gleason) P.J.F. Guim. \&

Michelang.

Andesanthus paleaceus (Triana ex Mart.) P.J.F.Guim. \& Michelang.

Pleroma pallidum (Cogn.) P. J. F. Guim. \& Michelang.

Chaetogastra panicularis Naudin

Chaetogastra paratropica (Griseb.) P.J.F. Guim. \&

Michelang.

Chaetogastra parviflora (Cogn.) P.J.F. Guim. \&

Michelang.

Chaetogastra patens (Todzia) P.J.F. Guim. \& Michelang. Pleroma pauloalvinii (Vinha) P. J. F. Guim. \&

Michelang.

Chaetogastra pendula (Cogn.) P.J.F. Guim. \& Michelang.

Chaetogastra pentamera (Ule) P.J.F. Guim. \& Michelang. Pleroma pereirae (Brade \& Markgr.) P. J. F. Guim. \& Michelang.

Pleroma pilosum (Cogn.) P. J. F. Guim. \& Michelang. Chaetogastra pleromoides (Naudin) P.J.F. Guim. \&

Michelang.

Chaetogastra pulcherrima (Gleason) P.J.F. Guim. \&

Michelang.

Chaetogastra purpurascens (Cogn.) P.J.F. Guim. \&

Michelang.

Chaetogastra purpusii (Brandegee) P.J.F. Guim. \&

Michelang.

Pleroma quartzophilum (Brade) P. J. F. Guim. \&

Michelang.

Pleroma raddianum (DC.) Gardner

Pleroma radula (Markgr.) P. J. F. Guim. \& Michelang.

Pleroma ramboi (Brade) P. J. F. Guim. \& Michelang.

Pleroma redivivum O.Berg ex Triana

Pleroma regelianum (Cogn.) P. J. F. Guim. \& Michelang. Pleroma andersregnellii P.J.F.Guim. \& Michelang.

Pleroma reitzii (Brade) P. J. F. Guim. \& Michelang. 
Tibouchina repens Wurdack

Tibouchina rhynchantherifolia Cogn.

Tibouchina riedeliana Cogn.

Tibouchina rigidula (Naudin) Wurdack

Tibouchina riparia Markgr.

Tibouchina robusta Cogn.

Tibouchina rojasii Cogn.

Tibouchina roseotincta Todzia

Tibouchina rubrobracteata R. Romero \& P.J.F.Guim.

Tibouchina rufipilis (Schltdl.) Cogn.

Tibouchina rupestris Cogn.

Tibouchina rupicola Hoehne

Tibouchina rusbyi Cogn.

Tibouchina salviifolia Cogn.

Tibouchina sandiensis Wurdack

Tibouchina saxicola F.S. Mey., P.J.F.Guim. \&

R.Goldenb.

Tibouchina saxosa Gleason

Tibouchina scaberrima Cogn.

Tibouchina scabriuscula (Schltdl.) Cogn.

Tibouchina schenckii Cogn.

Tibouchina schiedeana (Schltdl. \& Cham.) Cogn.

Tibouchina schwackei Cogn.

Tibouchina sebastianopolitana (Raddi) Cogn.

Tibouchina sellowiana (Cham.) Cogn.

Tibouchina semidecandra (Mart. \& Schrank ex DC.) Cogn.

Tibouchina sericea de Santiago

Tibouchina setoso-ciliata Cogn.

Tibouchina silvestris Todzia \& Almeda

Tibouchina simplicicaulis Cogn.

Tibouchina sinaloensis Todzia

Tibouchina solmsii Cogn.

Tibouchina stellipilis Wurdack
Chaetogastra repens (Wurdack) P.J.F. Guim. \& Michelang.

Chaetogastra rhynchantherifolia (Cogn.) P.J.F. Guim. \&

Michelang.

Pleroma riedelianum (Cogn.) P. J. F. Guim. \&

Michelang.

Pleroma rigidulum ((Naudin) Wurdack) P. J. F. Guim. \& Michelang.

Pleroma riparium (Markgr.) P. J. F. Guim. \& Michelang.

Pleroma robustm (Cogn.) P. J. F. Guim. \& Michelang.

Chaetogastra rojasii (Cogn.) P.J.F. Guim. \& Michelang.

Chaetogastra roseotincta (Todzia) P.J.F. Guim. \&

Michelang.

Pleroma rubrobracteatum (R.Romero \& P.J.F.Guim.) P.

J. F. Guim. \& Michelang.

Chaetogastra rufipilis (Schltdl.) Walp.

Chaetogastra rupestris (Cogn.) P.J.F. Guim. \&

Michelang.

Pleroma rupicola (Hoehne) P. J. F. Guim. \& Michelang.

Chaetogastra rusbyi (Cogn.) P.J.F. Guim. \& Michelang.

Pleroma salviaefolium (Cham.) Triana

Chaetogastra sandiensis (Wurdack) P.J.F. Guim. \&

Michelang.

Chaetogastra saxicola (F.S.Mey., P.J.F.Guim. \&

R.Goldenb.) P.J.F. Guim. \& Michelang.

Chaetogastra saxosa (Gleason) P.J.F. Guim. \&

Michelang.

Pleroma scaberrimum Triana

Chaetogastra scabriuscula (Schlecht.) P.J.F. Guim. \&

Michelang.

Pleroma schenckii (Cogn.) P. J. F. Guim. \& Michelang.

Chaetogastra schiedeana (Schltdl. \& Cham.) Walp.

Pleroma schwackei (Cogn.) P. J. F. Guim. \& Michelang. Chaetogastra sebastianapolitana (Raddi) P.J.F. Guim. \&

Michelang.

Pleroma sellowianum (Cham) P. J. F. Guim. \&

Michelang.

Pleroma semidecandrum (Schrank et Mart. ex DC.)

Triana

Chaetogastra sericea (de Santiago) P.J.F. Guim. \&

Michelang.

Pleroma setosociliatum (Cogn.) F.S.Mey. \& F.B.Matos Andesanthus silvestris (Todzia \& Almeda) P.J.F.Guim.\& Michelang.

Chaetogastra simplicicaulis (Naudin) P.J.F. Guim. \&

Michelang.

Chaetogastra sinaloensis (Todzia) P.J.F. Guim. \& Michelang.

Chaetogastra solmsii (Cogn.) P.J.F. Guim. \& Michelang.

Pleroma stellipile (Wurdack) P. J. F. Guim. \& Michelang. 
Tibouchina stenocarpa (DC.) Cogn.

Tibouchina stenopetala Cogn.

Tibouchina stenophylla Cogn.

Tibouchina stipulacea Vinha

Tibouchina strigosa (Rich.) Cogn

Tibouchina subglabra Wurdack

Tibouchina taperoensis Wurdack Tibouchina tedescoi Meirelles, L.Kollmann \& R.Goldenb.

Tibouchina tetrapetala Cogn.

Tibouchina thereminiana Cogn.

Tibouchina thulia Todzia

Tibouchina tomentulosa Wurdack

Tibouchina tortuosa (Bonpl.) Almeda

Tibouchina trichopoda (DC.) Baill.

Tibouchina triflora Gleason

Tibouchina trinitensis Cogn.

Tibouchina tuberosa Cogn.

Tibouchina urbanii Cogn.

Tibouchina urceolaris (Schrank \& Mart. ex DC.)

Cogn

Tibouchina ursina (Cham.) Cogn.

Tibouchina urvilleana (DC.) Cogn.

Tibouchina velutina Cogn.

Tibouchina venosa Gleason

Tibouchina versicolor Cogn.

Tibouchina villosissima Cogn.

Tibouchina viminea Cogn.

Tibouchina violacea Cogn.

Tibouchina virgata Cogn.

Tibouchina wasshausenii Wurdack

Tibouchina weberbaueri Cogn.

Tibouchina wurdackii Almeda \& Todzia

Tibouchinopsis glutinosa Markgr.
Pleroma stenocarpa (Schrank et Mart. ex DC.) Triana Chaetogastra stenopetala (Triana) P.J.F. Guim. \& Michelang.

Chaetogastra stenophylla (Cogn.) P.J.F. Guim. \&

Michelang.

Pleroma stipulaceum (Guimarães da Vinha) P. J. F.

Guim. \& Michelang.

Chaetogastra strigosa (Rich.) DC

Pleroma subglabrum (Wurdack) P. J. F. Guim. \&

Michelang.

Pleroma taperoense (Wurdack) P. J. F. Guim. \&

Michelang.

Pleroma tedescoi (Meirelles, L.Kollmann \& R.Goldenb.)

P.J.F. Guim. \& Michelang.

Chaetogastra tetrapetala (Cogn.) P.J.F. Guim. \&

Michelang.

Pleroma thereminianum (DC.) Triana

Chaetogastra thulia (Todzia) P.J.F. Guim. \& Michelang. Pleroma tomentulosum (Wurdack) P. J. F. Guim. \& Michelang.

Chaetogastra tortuosa (Bonpl.) DC.

Pleroma trichopodum DC.

Chaetogastra triflora (Gleason) P. J. F. Guim. \&

Michelang.

Chaetogastra trinitensis (Cogn.) P.J.F. Guim. \&

Michelang.

Pleroma tuberosum Gardner ex Triana

Chaetogastra urbanii (Cogn.) P.J.F. Guim. \& Michelang.

Pleroma urceolare (Schrank \& Mart. ex DC.) Triana

Pleroma ursinum (Cham.) Triana

Pleroma urvilleanum (DC.) P.J.F.Guim. \& Michelang.

Pleroma velutinum (Naud.) Triana

Chaetogastra venosa (Gleason) P.J.F. Guim. \&

Michelang.

Chaetogastra versicolor (Lindl.) P.J.F. Guim. \&

Michelang.

Pleroma villosissimum Mart. ex.Triana

Pleroma vimineum (D. Don) D. Don

Chaetogastra violacea (Cogn.) P.J.F. Guim. \& Michelang.

Pleroma virgatum Gardner

Chaetogastra wasshausenii (Wurdack) P.J.F. Guim. \&

Michelang.

Chaetogastra weberbaueri (Cogn.) P.J.F. Guim. \&

Michelang.

Andesanthus wurdackii (Almeda \& Todzia) P.J.F.Guim.

\& Michelang.

Pleroma glutinosum (Markgr.) P. J. F. Guim. \&

Michelang. 
Tibouchinopsis mirabilis Brade \& Markgr.

Tococa aristata Benth.

Tococa bolivarensis Gleason

Tococa broadwayi Urb.

Tococa bullifera Mart. \& Schrank ex DC.

Tococa capitata Trail ex Cogn.

Tococa caquetana Sprague

Tococa carolensis Gleason

Tococa caryophyllaea (DC.) S.S.Renner

Tococa caudata Markgr.

Tococa ciliata Triana

Tococa cinnamomea

Tococa cordata O.Berg ex Triana

Tococa coronata Benth.

Tococa costoides Michelangeli

Tococa croatii Almeda

Tococa desiliens Gleason

Tococa erioneura (Cogn.) Wurdack

Tococa erythrophylla (Ule) Wurdack

Tococa filiformis (Gleason) Wurdack

Tococa gonoptera Gleason

Tococa hirta O.Berg ex Triana

Tococa lancifolia Spruce ex Triana

Tococa leticiana Michelangeli

Tococa liesneri Wurdack

Tococa macrophysca Spruce ex Triana

Tococa macroptera Naudin

Tococa macrosperma Mart.

Tococa meridensis Wurdack

Tococa nitens (Benth.) Triana

Tococa obovata Gleason

Tococa oligantha Gleason

Tococa pachystachya Wurdack

Tococa pauciflora Spruce ex Triana

Tococa perclara Wurdack

Tococa platyphylla Benth.

Tococa quadrialata (Naudin) J.F.Macbr.

Tococa racemifera Wurdack

Tococa raggiana Michelangeli

Tococa rotundifolia (Triana) Wurdack
Pleroma mirabile (Brade \& Markgr.) P. J. F. Guim. \& Michelang.

Miconia tocoaristata Michelang.

Miconia canaimana Michelang.

Miconia broadwayii (Urb.) Michelang.

Miconia bullifera (Mart. Ex. Schrank ex DC.) Michelang.

Miconia tococapitata Michelang.

Miconia caquetana (Sprague) Michelang.

Miconia carolensis (Gleason) Michelang.

Miconia caryophyllea (DC.) Triana

Miconia tococaudata Michelang.

Miconia tocociliata Michelang.

Miconia tococinnamomea Michelang.

Miconia tococordata Michelang.

Miconia tococoronata Michelang.

Miconia costoides (Michelang.) Michelang.

Miconia croatii (Almeda) Michelang. \& Almeda

Miconia desiliens (Gleason) Michelang.

Miconia erioneura Cogn.

Miconia erythrophylla Ule

Miconia filiformis (Gleason) Michelang.

Miconia gonoptera (Gleason) Michelang.

Miconia tocohirta Michelang.

Miconia lancifolia (Spruce ex Triana) Michelang.

Miconia leticiana (Michelang.) Michelang.

Miconia ronliesneri Michelang.

Miconia macrophysca (Spruce ex Triana) Michelang.

Miconia macroptera (Naudin) Michelang.

Miconia macrosperma (Mart) Michelang.

Miconia norandina Michelang.

Miconia nitens Benth.

Miconia tocobovata Michelang.

Miconia setimarginata Pittier

Miconia pachystachya (Wurdack) Michelang.

Miconia tocopauciflora Michelang.

Miconia perclara (Wurdack) Michelang.

Miconia platyphylla (Benth.) L.O.Williams

Miconia microphysca Michelang.

Miconia tocoracemifera Michelang.

Miconia raggiana (Michelang.) Michelang.

Miconia rubripetala Michelang. 
Tococa setifera Pilg.

Tococa spadiciflora Triana

Tococa stellata Gleason

Tococa stephanotricha Naudin

Tococa subciliata (DC.) Triana

Tococa symphyandra (Triana) Cogn.

Tococa tepuiensis Wurdack

Tococa undabunda J. F.Macbr.

Topobea acuminata Wurdack

Topobea adscendens E.Cotton \& Matezki

Topobea aeruginosa (Standl.) L.O.Williams

Topobea albertiae Wurdack

Topobea alternifolia Gleason

Topobea amplifolia Almeda

Topobea andreana Cogn.

Topobea anisophylla Triana

Topobea arboricola Almeda

Topobea asplundii Wurdack

Topobea barbata Gleason

Topobea brachyura (Gleason) Wurdack

Topobea brenesii Standl.

Topobea brevibractea Gleason

Topobea bullata E.Cotton \& Matezki

Topobea calcarata L.Uribe

Topobea caliginosa Almeda

Topobea calophylla Almeda

Topobea calycularis Naudin

Topobea castanedae Wurdack

Topobea caudata Wurdack

Topobea ciliata Cogn.

Topobea cordata Gleason

Topobea crassifolia (Almeda) Almeda

Topobea cutucuensis Wurdack

Topobea dimorphophylla Almeda

Topobea discolor Hochr.

Topobea dodsonorum Wurdack

Topobea eplingii Wurdack

Topobea ferruginea Gleason

Topobea fragrantissima Almeda
Miconia setifera (Pilg.) Michelang.

Miconia spadiciflora (Triana) Michelang.

Miconia stenoptera (Gleason) Michelang.

Miconia stephanotricha (Naudin) Michelang.

Miconia subciliata DC.

Miconia symphyadra Triana

Miconia tepuiensis (Wurdack) Michelang

Miconia undabunda (J. F. Macbr.) Michelang.

Blakea acuminata (Wurdack) Penneys \& Judd

Blakea adscendens (E. Cotton \& Matezki) Penneys \& Judd

Blakea aeruginosa Standl.

Blakea albertiae (Wurdack) Penneys \& Judd

Blakea alternifolia (Gleason) Gleason

Blakea amplifolia (Almeda) Penneys \& Almeda

Blakea pectinata Penneys, Phytoneuron

0.91666666666666674

Blakea anisophylla (Triana) Baill.

Blakea arboricola (Almeda) Penneys \& Almeda

Blakea asplundii (Wurdack) Penneys \& Judd

Blakea barbata (Gleason) Penneys \& Judd

Blakea brachyura Gleason

Blakea brenesii (Standl.) Penneys \& Almeda

Blakea brevibractea (Gleason) Penneys \& Judd

Blakea bullata (E.Cotton \& Matezki) Penneys \& Judd

Blakea calcarata (L. Uribe) Penneys \& Judd

Blakea micrantha Almeda

Blakea calophylla (Almeda) Penneys \& Almeda

Blakea calycularis (Naudin) Penneys \& Almeda

Blakea castanedae (Wurdack) Penneys \& Judd

Blakea horologica Penneys \& Judd

Blakea ciliata Markgr.

Blakea cordata (Gleason) Penneys \& Almeda

Blakea crassifolia Almeda

Blakea cutucuensis (L. Uribe) Penneys \& Judd

Blakea dimorphophylla (Almeda) Penneys \& Almeda

Blakea discolor (Hochr.) Penneys \& Judd,

Blakea dodsoniorum (Wurdack) Penneys \& Almeda

Blakea eplingii (Wurdack) Penneys \& Judd,

Blakea ferruginea (Gleason) Penneys \& Judd

Blakea fragrantissima (Almeda) Penneys \& Almeda 
Topobea gerardoana Almeda

Topobea glaberrima Triana

Topobea glabrescens Triana

Topobea gracilis Triana

Topobea hexandra Almeda

Topobea induta Markgr.

Topobea inflata Triana

Topobea insignis Triana

Topobea intricata Almeda

Topobea killipii Wurdack

Topobea laevigata (D.Don) Naudin

Topobea lentii Almeda

Topobea longiloba Wurdack

Topobea longisepala Gleason

Topobea macbrydei Wurdack

Topobea maguirei Wurdack

Topobea maurofernandeziana Cogn.

Topobea mcphersonii Almeda

Topobea micrantha Pittier

Topobea modica Wurdack

Topobea mortoniana Wurdack

Topobea multiflora (D.Don) Triana

Topobea muricata Lozano

Topobea parasitica Aubl.

Topobea parvifolia (Gleason) Almeda

Topobea pascoensis Wurdack

Topobea pittieri Cogn.

Topobea pluvialis Standl.

Topobea punctulata Triana

Topobea rotundifolia (D.Don) Naudin

Topobea sessilifolia Triana

Topobea setosa Triana

Topobea standleyi L.O.Williams

Topobea stephanochaeta Naudin

Topobea steyermarkii Wurdack

Topobea storkiii Standl.

Topobea suaveolens Almeda

Topobea subbarbata Wurdack

Topobea subscabrula Triana

Topobea subsessiliflora Wurdack
Blakea gerardoana (Almeda) Penneys \& Almeda

Blakea glaberrima (Triana) Penneys \& Judd

Blakea cuprina Penneys \& Judd

Blakea clavata Penneys \& Judd

Blakea hexandra (Almeda) Penneys \& Almeda

Blakea induta (Markgr.) Penneys \& Judd

Blakea inflata (Triana) Penneys \& Judd

Blakea insignis (Triana) Penneys \& Judd

Blakea intricata (Almeda) Penneys \& Almeda

Blakea killipii (Wurdack) Penneys \& Judd

Blakea laevigata D. Don

Blakea lentii (Almeda) Penneys \& Almeda

Blakea longiloba (Wurdack) Penneys \& Judd

Blakea longisepala (Gleason) Penneys \& Judd

Blakea macbrydei (Wurdack) Penneys \& Judd

Blakea maguirei (Wurdack) Penneys \& Judd

Blakea maurofernandeziana (Cogn.) Penneys \& Almeda

Blakea mcphersonii (Almeda) Penneys \& Almeda

Miconia concinna Almeda

Blakea modica (Wurdack) Penneys \& Judd

Blakea mortoniana (Wurdack) Penneys \& Judd

Blakea multiflora D.Don

Blakea muricata (Lozano) Penneys \& Judd

Blakea parasitica (Aublet) D.Don

Blakea parvifolia Gleason

Blakea pascoensis (Wurdack) Penneys \& Judd

Blakea henripittieri Penneys \& Almeda

Blakea pluvialis (Standl.) Penneys \& Almeda

Blakea punctulata (Triana) Wurdack

Blakea rotundifolia D.Don

Blakea sessilifolia (Triana) Penneys \& Judd

Blakea setosa (Triana) Penneys \& Judd

Blakea standleyi (L.O.Williams) Penneys \& Almeda

Blakea stephanochaeta (Naudin) Penneys \& Judd

Blakea steyermarkii (Wurdack) Penneys \& Judd

Blakea storkii (Standl.) Almeda

Blakea suaveolens (Almeda) Penneys \& Almeda

Blakea subbarbata (Wurdack) Penneys \& Judd

Blakea subscabrula (Triana) Penneys \& Judd

Blakea subsessiliflora (Wurdack) Penneys \& Judd 
Topobea superba Naudin

Topobea tetramera Almeda

Topobea tetroici Wurdack

Topobea toachiensis Wurdack

Topobea trianaei Cogn.

Topobea verrucosa Wurdack

Topobea watsonii Cogn.
Blakea superba (Naudin) Penneys \& Judd

Blakea tetramera (Almeda) Penneys \& Almeda

Blakea tetroici (Wurdack) Penneys \& Judd

Blakea toachiensis (Wurdack) Penneys \& Judd

Blakea trianae (Cogn.) Penneys \& Judd

Blakea verrucosa (Wurdack) Penneys \& Judd

Blakea watsonii (Cogn.) Penneys \& Almeda 
Table 5: Currently accepted species of New World Melastomataceae (as of October 15, 2020).

Acanthella pulchra Gleason

Acanthella sprucei Benth. \& Hook. f.

Aciotis acuminifolia (Mart. ex DC.) Triana

Aciotis annua (Mart. ex DC.) Triana

Aciotis circaeifolia (Bonpl.) Triana

Aciotis ferreirana Brade

Aciotis indecora (Bonpl.) Triana

Aciotis olivieriana Freire-Fierro

Aciotis ornata (Miq.) Gleason

Aciotis paludosa (Mart. ex DC.) Triana

Aciotis polystachya (Bonpl.) Triana

Aciotis purpurascens (Aubl.) Triana

Aciotis rubricaulis (Mart. ex DC.) Triana

Aciotis viscida (Benth.) Freire-Fierro

Aciotis wurdackiana Freire-Fierro

Acisanthera alsinaefolia (DC.) Triana

Acisanthera ayangannae (Wurdack) M.J.R.Rocha \& P.J.F.Guim.,

Acisanthera boliviensis Cogn. ex Kuntze

Acisanthera erecta J.St.-Hil.

Acisanthera fluitans Cogn.

Acisanthera paraguayensis (Hook.f.) Cogn .

Acisanthera uniflora (Vahl) Gleason

Acisanthera variabilis (DC.) Triana

Adelobotrys acreana Wurdack

Adelobotrys adscendens (Sw.) Triana

Adelobotrys atlantica Schulman

Adelobotrys ayangannensis Wurdack

Adelobotrys barbata Triana

Adelobotrys boissierianus Cogn.

Adelobotrys ciliatus (Naudin) Triana

Adelobotrys duidae (Gleason) Wurdack

Adelobotrys fruticosa Wurdack

Adelobotrys fuscescens Triana

Adelobotrys intonsus (Gleason) Wurdack

Adelobotrys jefensis Almeda

Adelobotrys klugii Wurdack

Adelobotrys latifolius Schulman

Adelobotrys linearifolius L. Uribe

Adelobotrys macrantha Gleason

Adelobotrys macrophyllus Pilg.

Adelobotrys marginatus Brade

Adelobotrys microcarpus Schulman

Adelobotrys monticola Gleason

Adelobotrys permixta Wurdack

Adelobotrys praetextus Pilg.

Adelobotrys rachidotrichia Brade

Adelobotrys rotundifolius Triana

Adelobotrys ruokolainenii Schulman

Adelobotrys saxosa Wurdack

Adelobotrys scandens (Aubl.) DC.

Adelobotrys spruceanus Cogn.

Adelobotrys stenophylla Wurdack

Adelobotrys subsessilis Gleason 
Adelobotrys tessmannii Markgr.

Allomaieta caucana Lozano

Allomaieta ebejicosana Lozano

Allomaieta grandiflora Gleason

Allomaieta hirsuta (Gleason) Lozano

Allomaieta javierbarrigae $\mathrm{H}$. Mend.

Allomaieta pancurana Lozano

Allomaieta strigosa (Gleason) Lozano

Allomaieta villosa (Gleason) Lozano

Allomaieta zenufanasana Lozano

Alloneuron glomeratum C. Ulloa \& Michelang.

Alloneuron liron B. Walln.

Alloneuron majus (Markgr.) Markgr. ex J.F. Macbr.

Alloneuron ronliesneri B. Walln.

Alloneuron ulei Pilg.

Andesanthus aristeguietae (Wurdack) P.J.F.Guim. \& Michelang.

Andesanthus elegantulus (Todzia \& Almeda) P.J.F.Guim.\& Michelang.

Andesanthus gleasonianus (Wurdack) P.J.F.Guim. \& Michelang.

Andesanthus inopinatus (Wurdack) P.J.F.Guim. \& Michelang.

Andesanthus lepidotus (Bonpl.) P.J.F.Guim. \& Michelang.

Andesanthus narinoensis (Wurdack) P.J.F.Guim. \& Michelang.

Andesanthus paleaceus (Triana ex Mart.) P.J.F.Guim. \& Michelang.

Andesanthus silvestris (Todzia \& Almeda) P.J.F.Guim.\& Michelang.

Andesanthus wurdackii (Almeda \& Todzia) P.J.F.Guim. \& Michelang.

Appendicularia pullei (Gleason) M.J.R.Rocha \& P.J.F.Guim

Appendicularia subglabra (Wurdack) M.J.R.Rocha \& P.J.F.Guim.

Appendicularia thymifolia (Bonpl.) DC.

Arthrostemma alatum Triana

Arthrostemma ciliatum Pav. ex D. Don

Arthrostemma parvifolium Cogn.

Arthrostemma primaevum Almeda

Axinaea affinis (Naudin) Cogn.

Axinaea alata E. Cotton

Axinaea campii E. Cotton

Axinaea carolina-telleziae Bussmann \& Paniagua

Axinaea colombiana Lozano \& Alvear

Axinaea confusa E. Cotton

Axinaea costaricensis Cogn.

Axinaea crassinoda Triana

Axinaea dentata E. Cotton

Axinaea dependens Ruiz \& Pav. ex D. Don

Axinaea disrupta E. Cotton

Axinaea fallax Gleason

Axinaea fernando-cabiesii Bussmann, J.A. Gruhn \& A. Glenn

Axinaea flava E. Cotton, Bussmann \& P. Lozano

Axinaea floribunda (Naudin) Triana

Axinaea glandulosa Ruiz \& Pav. ex D. Don

Axinaea glauca E. Cotton \& P. Lozano

Axinaea grandifolia (Naudin) Triana

Axinaea lanceolata Ruiz \& Pav.

Axinaea lawessonii E. Cotton

Axinaea lehmannii Cogn.

Axinaea luteynii E. Cotton

Axinaea macrophylla (Naudin) Triana

Axinaea merianiae (DC.) Triana

Axinaea mertensioides Wurdack 


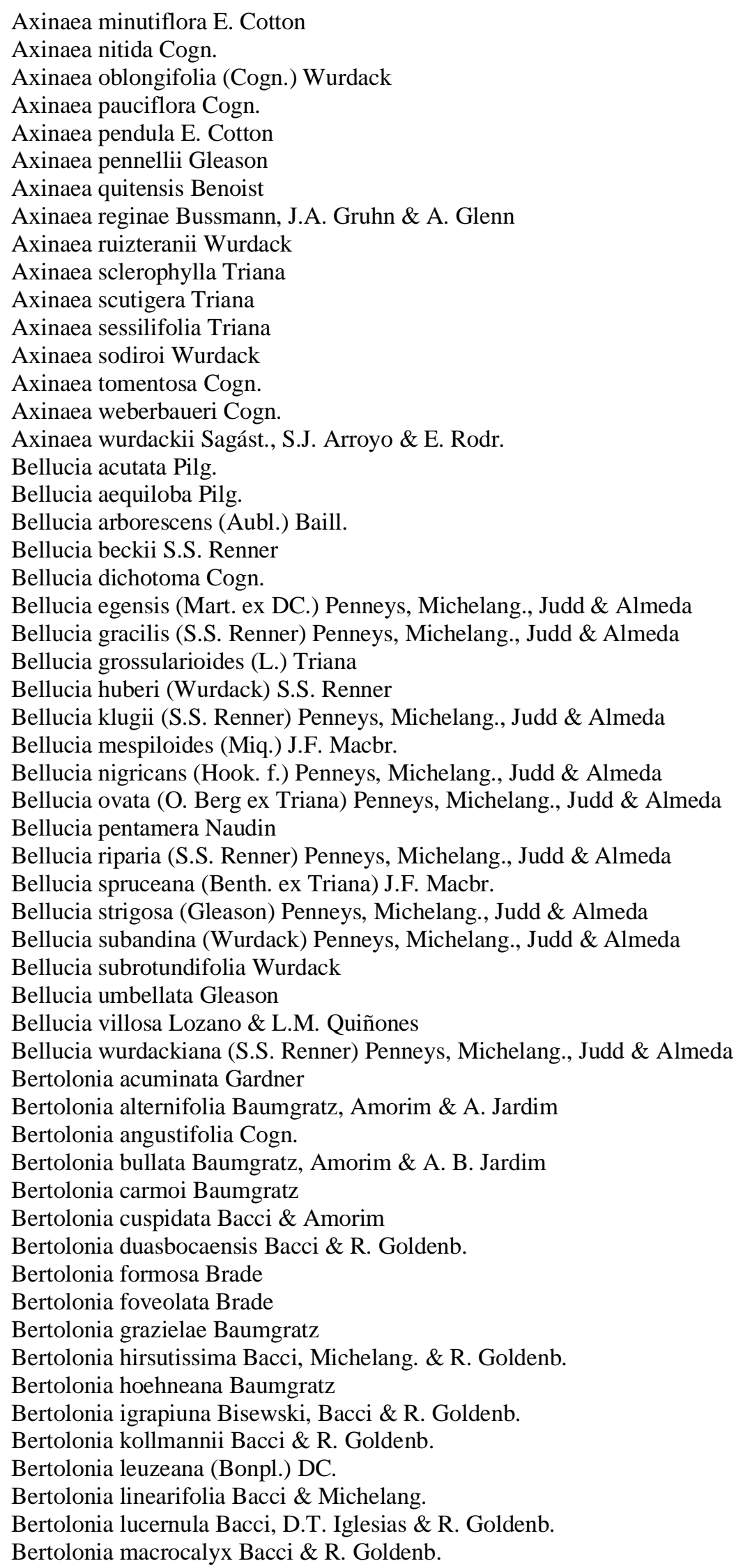


Bertolonia maculata DC.

Bertolonia margaritacea Naudin

Bertolonia marmorata (Naudin) Naudin

Bertolonia michelangeliana Bacci \& R. Goldenb.

Bertolonia mosenii Cogn.

Bertolonia nymphaeifolia Raddi

Bertolonia organensis Baumgratz, Silva-Gonçalves \& Nunes-Freitas

Bertolonia paranaensis Baumgratz

Bertolonia reginatoi Bacci \& Michelang.

Bertolonia riocontensis Bisewski, Bacci \& R. Goldenb.,

Bertolonia ruschiana Bacci \& R. Goldenb.

Bertolonia sanguinea Saldanha ex Cogn.

Bertolonia valenteana Baumgratz

Bertolonia venezuelensis Wurdack

Bertolonia violacea Bisewski, Bacci \& R. Goldenb.,

Bertolonia vitoriana Bacci \& Amorim

Bertolonia wurdackiana Baumgratz

Bisglaziovia behurioides Cogn.

Blakea acostae Wurdack

Blakea acuminata (Wurdack) Penneys \& Judd

Blakea adscendens (E. Cotton \& Matezki) Penneys \& Judd

Blakea aeruginosa Standl.

Blakea albertiae (Wurdack) Penneys \& Almeda

Blakea allotricha L. Uribe

Blakea alternifolia (Gleason) Gleason

Blakea amabilis (Linden) Cogn.

Blakea amplifolia (Almeda) Penneys \& Almeda

Blakea andreana Cogn.

Blakea anisophylla (Triana) Baill.

Blakea anomala Donn. Sm.

Blakea arboricola (Almeda) Penneys \& Almeda

Blakea argentea Gleason

Blakea asplundii (Wurdack) Penneys \& Judd

Blakea attenboroughii Penneys \& L. Jost

Blakea austin-smithii Standl.

Blakea barbata (Gleason) Penneys \& Judd

Blakea bocatorena Kriebel \& D. Santam.

Blakea brachyura Gleason

Blakea bracteata Gleason

Blakea brasiliensis Cogn.

Blakea brenesii (Standl.) Penneys \& Almeda

Blakea brevibracteata (Gleason) Penneys \& Judd

Blakea brunnea Gleason

Blakea bullata (E. Cotton \& Matezki) Penneys \& Judd

Blakea calcarata (L. Uribe) Penneys \& Judd

Blakea calophylla (Almeda) Penneys \& Almeda

Blakea calycosa Gleason

Blakea calycularis (Naudin) Penneys \& Almeda

Blakea calyptrata Gleason

Blakea campii Wurdack

Blakea castanedae (Wurdack) Penneys \& Judd

Blakea chlorantha Almeda

Blakea ciliata Markgr.

Blakea clavata Penneys \& Judd

Blakea clusiifolia Gleason

Blakea coloradensis Almeda 
Blakea cordata (Gleason) Penneys \& Almeda

Blakea costaricensis Umaña \& Almeda

Blakea crassifolia Almeda

Blakea crinita Gleason

Blakea cuatrecasasii Gleason

Blakea cuneata Standl.

Blakea cuprina Penneys \& Judd

Blakea cutucuensis (Wurdack) Penneys \& Judd

Blakea darcyana Almeda

Blakea dimorphophylla (Almeda) Penneys \& Almeda

Blakea discolor (Hochr.) Penneys \& Judd

Blakea dodsoniorum (Wurdack) Penneys \& Almeda

Blakea droseripila Penneys

Blakea echinata Almeda \& Penneys

Blakea elliptica (Gleason) Almeda

Blakea eplingii (Wurdack) Penneys \& Judd

Blakea eriocalyx Wurdack

Blakea fasciculata Gleason

Blakea ferruginea (Gleason) Penneys \& Judd

Blakea fissicalyx L. Uribe

Blakea florifera Gleason

Blakea foliacea Gleason

Blakea formicaria Wurdack

Blakea fragrantissima (Almeda) Penneys \& Almeda

Blakea fuchsioides Almeda

Blakea gerardoana (Almeda) Penneys \& Almeda

Blakea glaberrima (Triana) Penneys \& Judd

Blakea glabrescens Benth.

Blakea glandulosa Gleason

Blakea gracilis Hemsl.

Blakea granatensis Naudin

Blakea grandiflora Hemsl.

Blakea gregii Almeda

Blakea grisebachii Cogn.

Blakea guatemalensis Donn. Sm.

Blakea hammelii Almeda

Blakea hammettiorum Almeda

Blakea harlingii Wurdack

Blakea henripittieri Penneys \& Almeda

Blakea herrerae Almeda

Blakea hexandra (Almeda) Penneys \& Almeda

Blakea hirsuta Berg ex Triana

Blakea hirsutissima (J.F. Macbr.) Wurdack

Blakea hispida Markgr.

Blakea holtonii Hochr.

Blakea horologica Penneys \& Judd

Blakea hydraeformis Wurdack

Blakea incompta Markgr.

Blakea induta (Markgr.) Penneys \& Judd

Blakea inflata (Triana) Penneys \& Judd

Blakea insignis (Triana) Penneys \& Judd

Blakea intricata (Almeda) Penneys \& Almeda

Blakea involvens Markgr.

Blakea jativae Wurdack

Blakea killipii (Wurdack) Penneys \& Judd

Blakea laevigata D. Don 
Blakea lanuginosa Wurdack

Blakea latifolia (Ruiz \& Pav.) D. Don

Blakea lentii (Almeda) Penneys \& Almeda

Blakea lindeniana (Naudin) Triana

Blakea litoralis L.O. Williams

Blakea longibracteata Cogn.

Blakea longiloba (Wurdack) Penneys \& Judd

Blakea longipes L. Uribe

Blakea longisepala (Gleason) Penneys \& Judd

Blakea macbrydei (Wurdack) Penneys \& Judd

Blakea madisonii Wurdack

Blakea maguirei (Wurdack) Penneys \& Judd

Blakea maurofernandeziana (Cogn.) Penneys \& Almeda

Blakea mcphersonii (Almeda) Penneys \& Almeda

Blakea megaphylla Wurdack

Blakea mexiae Gleason

Blakea micrantha Almeda

Blakea modica (Wurdack) Penneys \& Judd

Blakea monticola J.R. Johnst.

Blakea mortoniana (Wurdack) Penneys \& Judd

Blakea multiflora D. Don

Blakea muricata (Lozano) Penneys \& Judd

Blakea nangaritzana D. Fernández, C. Ulloa \& Penneys

Blakea nareliana Bussmann

Blakea nodosa Wurdack

Blakea oldemanii Wurdack

Blakea orientalis Gleason

Blakea ovalis (Ruiz \& Pav.) D. Don

Blakea paleacea Gleason

Blakea paludosa Gleason

Blakea parasitica (Aubl.) D. Don

Blakea parvifolia Gleason

Blakea pascoensis (Wurdack) Penneys \& Judd

Blakea pauciflora Gleason

Blakea pectinata Penneys

Blakea penduliflora Almeda

Blakea perforata Almeda

Blakea pichinchensis Wurdack

Blakea pilosa Gleason

Blakea platypoda Gleason

Blakea pluvialis (Standl.) Penneys \& Almeda

Blakea podagrica Triana

Blakea polyantha Wurdack

Blakea portentosa Wurdack

Blakea princeps (Linden ex Mast.) Cogn.

Blakea pulverulenta Vahl

Blakea punctulata (Triana) Wurdack

Blakea purpusii Brandegee

Blakea pyxidanthus Triana

Blakea quadrangularis Triana

Blakea quadriflora Gleason

Blakea repens (Ruiz \& Pav.) D. Don

Blakea ricardoi Michelang. \& D.Santam.

Blakea rosea (Ruiz \& Pav.) D. Don

Blakea rostrata Berg ex Triana

Blakea rotundifolia D. Don 
Blakea sawadae J.F. Macbr.

Blakea scarlatina Almeda

Blakea schlimii (Naudin) Triana

Blakea schultzei Markgr.

Blakea sessilifolia (Triana) Penneys \& Judd

Blakea setosa (Triana) Penneys \& Judd

Blakea spruceana Cogn.

Blakea squamigera L. Uribe

Blakea standleyana J.F. Macbr.

Blakea standleyi (L.O. Williams) Penneys \& Almeda

Blakea stellaris Gleason

Blakea stephanochaeta (Naudin) Penneys \& Judd

Blakea steyermarkii (Wurdack) Penneys \& Judd

Blakea stipulacea Wurdack

Blakea storkii (Standl.) Almeda

Blakea suaveolens (Almeda) Penneys \& Almeda

Blakea subbarbata (Wurdack) Penneys \& Judd

Blakea subconnata O. Berg ex Triana

Blakea subpanduriformis E. Cotton \& Matezki

Blakea subscabrula (Triana) Penneys \& Judd

Blakea subsessiliflora (Wurdack) Penneys \& Judd

Blakea subvaginata Wurdack

Blakea superba (Naudin) Penneys \& Judd

Blakea tapantiana Umaña \& Almeda

Blakea tetramera (Almeda) Penneys \& Almeda

Blakea tetroici (Wurdack) Penneys \& Judd

Blakea toachiensis (Wurdack) Penneys \& Judd

Blakea trianae (Cogn.) Penneys \& Judd

Blakea trinervia L.

Blakea truncata Gleason

Blakea tuberculata Donn. Sm.

Blakea unguiculata Almeda \& Penneys

Blakea urbaniana Cogn.

Blakea vallensis Wurdack

Blakea venusta Kriebel, Almeda \& A. Estrada

Blakea verrucosa (Wurdack) Penneys \& Judd

Blakea villosa Cogn.

Blakea watsonii (Cogn.) Penneys \& Almeda

Blakea wilburiana Almeda

Blakea wilsoniorum Almeda

Boyania ayangannae Wurdack

Boyania colombiana H. Mend.

Brachyotum alpinum Cogn.

Brachyotum andreanum Cogn.

Brachyotum angustifolium Wurdack

Brachyotum azuayense Wurdack

Brachyotum barbeyanum Cogn.

Brachyotum benthamianum Triana

Brachyotum campanulare (Bonpl.) Triana

Brachyotum campii Wurdack

Brachyotum cernuum (Bonpl.) Triana

Brachyotum cogniauxii Wurdack

Brachyotum confertum (Bonpl.) Triana

Brachyotum coronatum (Triana) Wurdack

Brachyotum cutervoanum Wurdack

Brachyotum ecuadorense Wurdack 
Brachyotum fictum Wurdack

Brachyotum figueroae J.F. Macbr.

Brachyotum fraternum Wurdack

Brachyotum gleasonii Wurdack

Brachyotum gracilescens Triana

Brachyotum grisebachii Cogn.

Brachyotum harlingii Wurdack

Brachyotum huancavelicae Wurdack

Brachyotum incrassatum E. Cotton

Brachyotum intermedium Wurdack

Brachyotum jamesonii Triana

Brachyotum johannes-julii E. Cotton

Brachyotum ledifolium (Desr.) Triana

Brachyotum lindenii Cogn.

Brachyotum longisepalum Wurdack

Brachyotum lutescens (Ruiz \& Pav.) Triana

Brachyotum lycopodioides Triana

Brachyotum lymphatum Wurdack

Brachyotum markgrafii Wurdack

Brachyotum maximowiczii Cogn.

Brachyotum microdon (Naudin) Triana

Brachyotum multinervium Wurdack

Brachyotum multituberculatum Wurdack

Brachyotum naudinii Triana

Brachyotum nutans Gleason

Brachyotum parvifolium Cogn.

Brachyotum quinquenerve (Ruiz \& Pav.) Triana

Brachyotum radula Triana

Brachyotum rosmarinifolium (Ruiz \& Pav.) Triana

Brachyotum rostratum (Naudin) Triana

Brachyotum rotundifolium Cogn.

Brachyotum rugosum Wurdack

Brachyotum russatum E. Cotton

Brachyotum sanguinolentum (Naudin) Triana

Brachyotum sertulatum C. Ulloa

Brachyotum strigosum (L. f.) Triana

Brachyotum trichocalyx Triana

Brachyotum tyrianthinum J.F. Macbr.

Brachyotum uribei Wurdack

Brachyotum virescens (Cogn.) Wurdack

Brachyotum weberbaueri Cogn.

Brasilianthus carajensis Almeda \& Michelang.

Bucquetia glutinosa (L. f.) DC.

Bucquetia nigritella (Naudin) Triana

Bucquetia vernicosa Gleason

Cambessedesia angelana Fidanza \& Almeda

Cambessedesia atropurpurea A.B. Martins

Cambessedesia cambessedesioides (Wurdack) A.B. Martins

Cambessedesia corymbosa Mart. \& Schrank ex DC.

Cambessedesia eichleri Cogn.

Cambessedesia espora (A. St.-Hil. ex Bonpl.) DC.

Cambessedesia glaziovii Cogn. ex A.B. Martins

Cambessedesia gracilis Wurdack

Cambessedesia harleyi Wurdack

Cambessedesia hermogenesii A.B. Martins

Cambessedesia hilariana (Kunth) DC. 
Cambessedesia latevenosa Mart. ex DC.

Cambessedesia membranacea Gardner

Cambessedesia pityrophylla (DC.) A.B. Martins

Cambessedesia purpurata Schrank \& Mart. ex DC.

Cambessedesia regnelliana Cogn.

Cambessedesia rupestris A.B. Martins

Cambessedesia salviifolia (Cham.) A.B. Martins

Cambessedesia semidecandra A. St.-Hil. ex A.B. Martins

Cambessedesia striatella (Naudin) A.B. Martins

Cambessedesia tenuis Markgr.

Cambessedesia tiradentensis R.J.V. Alves, Rutter, R. A. \& A.B. Martins

Cambessedesia uncinata Fidanza \& Almeda

Cambessedesia weddellii Naudin

Cambessedesia wurdackii A.B. Martins

Castratella piloselloides Naudin

Castratella rosea Gleason

Centradenia floribunda Planch.

Centradenia grandifolia (Schltdl.) Endl.

Centradenia inaequilateralis (Schltdl. \& Cham.) G. Don

Centradenia paradoxa (Kraenzl.) Almeda

Centradeniastrum album Gleason

Centradeniastrum roseum Cogn.

Centronia laurifolia D. Don

Centronia neblinae Wurdack

Centronia reticulata Triana

Centronia sessilifolia Cogn.

Centronia vaupesana Wurdack

Chaetogastra adenophora (Cogn.) P.J.F. Guim. \& Michelang.

Chaetogastra alata P.J.F.Guim. \& Michelang.

Chaetogastra almedae (Todzia) P.J.F. Guim. \& Michelang.

Chaetogastra anderssonii (Wurdack) P.J.F. Guim. \& Michelang.

Chaetogastra andreana (Cogn.) P.J.F. Guim. \& Michelang.

Chaetogastra araneicalyx (de Santiago) P.J.F. Guim. \& Michelang.

Chaetogastra arthrostemmoides (Cogn.) P.J.F. Guim. \& Michelang.

Chaetogastra asperifolia (Cogn.) P.J.F. Guim. \& Michelang.

Chaetogastra aurea (Cogn.) P.J.F. Guim. \& Michelang.

Chaetogastra brittoniana (Cogn. ex Rusby) P.J.F. Guim. \& Michelang.

Chaetogastra campii (Wurdack) P.J.F. Guim. \& Michelang.

Chaetogastra capitata (Naudin) P.J.F. Guim. \& Michelang.

Chaetogastra cerastifolia (Naudin) P.J.F. Guim. \& Michelang.

Chaetogastra chamaecistus Sieber ex Grisebach

Chaetogastra chironioides Grisebach

Chaetogastra ciliaris DC.

Chaetogastra cisplatensis (Cogn.) P.J.F. Guim. \& Michelang.

Chaetogastra cistoides Griseb.

Chaetogastra citrina (Naudin) P.J.F. Guim. \& Michelang.

Chaetogastra clinopodifolia DC.

Chaetogastra confertiflora (Naudin) P.J.F. Guim. \& Michelang.

Chaetogastra congestiflora (Todzia) P.J.F. Guim. \& Michelang.

Chaetogastra connata (Gleason ex Todzia) P.J.F. Guim. \& Michelang.

Chaetogastra cordeiroi F.S. Mey. \& R. Goldenb.

Chaetogastra cornuta (Gleason) P.J.F. Guim. \& Michelang.

Chaetogastra crassilfolia F.S.Mey. \& R.Goldenb.

Chaetogastra cristaensis F.S.Mey. \& R.Goldenb.

Chaetogastra debilis Cham.

Chaetogastra decora (Gleason) P.J.F. Guim. \& Michelang. 
Chaetogastra dimorphylla (Gleason) P.J.F. Guim. \& Michelang. Chaetogastra durangensis (Standl.) P.J.F. Guim. \& Michelang. Chaetogastra echinata (Ruiz et Pav.) DC.

Chaetogastra erioclada (Triana) P.J.F. Guim. \& Michelang. Chaetogastra excoriata (Cogn.) P.J.F. Guim. \& Michelang. Chaetogastra ferrariana (Cogn.) P.J.F. Guim. \& Michelang. Chaetogastra fulvipilis (Cogn.) P.J.F. Guim. \& Michelang. Chaetogastra galeottiana (Naudin) P.J.F. Guim. \& Michelang. Chaetogastra gayana (Naudin) P.J.F. Guim. \& Michelang. Chaetogastra geitneriana Schltdl.

Chaetogastra gracilis DC.

Chaetogastra grossa (L. f.) P.J.F. Guim. \& Michelang. Chaetogastra hassleri (Cogn.) P.J.F. Guim. \& Michelang. Chaetogastra herbacea (DC.) P.J.F. Guim. \& Michelang. Chaetogastra herincquiana (Cogn.) P.J.F. Guim. \& Michelang. Chaetogastra herzogii (Cogn.) P.J.F. Guim. \& Michelang. Chaetogastra hieracioides DC.

Chaetogastra hintonii Gleason ex P.J.F. Guim. \& Michelang. Chaetogastra hutchisonii (Wurdack) P.J.F. Guim. \& Michelang. Chaetogastra incarum (Gleason) P.J.F. Guim. \& Michelang. Chaetogastra inconstans (Vahl.) P. J. F. Guim. \& Michelang. Chaetogastra kingii (Wurdack) P.J.F. Guim. \& Michelang. Chaetogastra kunhardtii (Gleason) P.J.F. Guim. \& Michelang. Chaetogastra laevis (Cogn.) P.J.F. Guim. \& Michelang. Chaetogastra lancifolia (Wurdack) P.J.F. Guim. \& Michelang. Chaetogastra latibracteolata (P.G.Wilson) P.J.F. Guim. \& Michelang. Chaetogastra latifolia (Naudin) P.J.F. Guim. \& Michelang. Chaetogastra laxa (Desr.) P.J.F. Guim. \& Michelang.

Chaetogastra longifolia (Vahl.) DC.

Chaetogastra longipilosa (Cogn.) P.J.F. Guim. \& Michelang.

Chaetogastra longisepala (Cogn.) P.J.F. Guim. \& Michelang.

Chaetogastra longistyla (Cogn.) P.J.F. Guim. \& Michelang.

Chaetogastra macvaughii (Todzia) P.J.F. Guim. \& Michelang.

Chaetogastra mariae (Wurdack) P.J.F. Guim. \& Michelang.

Chaetogastra membranifolia (Cogn. \& Rusby) P.J.F. Guim. \& Michelang.

Chaetogastra minor (Cogn.) P.J.F. Guim. \& Michelang.

Chaetogastra mollis (Bonpl.) DC.

Chaetogastra montana P.J.F. Guim. \& Michelang.

Chaetogastra monticola (Naudin) P.J.F. Guim. \& Michelang.

Chaetogastra nanifolia (Todzia) P.J.F. Guim. \& Michelang.

Chaetogastra naudiniana Decne.

Chaetogastra nitida (Graham) P.J.F. Guim. \& Michelang.

Chaetogastra obtusifolia (Cogn.) P.J.F. Guim. \& Michelang.

Chaetogastra oligantha (Gleason) P.J.F. Guim. \& Michelang.

Chaetogastra orbignyana (Naudin) P.J.F. Guim. \& Michelang.

Chaetogastra oroensis (Gleason) P.J.F. Guim. \& Michelang.

Chaetogastra panicularis Naudin

Chaetogastra paratropica (Griseb.) P.J.F. Guim. \& Michelang.

Chaetogastra parviflora (Cogn.) P.J.F. Guim. \& Michelang.

Chaetogastra patens (Todzia) P.J.F. Guim. \& Michelang.

Chaetogastra pauciflora P.J.F. Guim. \& Michelang.

Chaetogastra pendula (Cogn.) P.J.F. Guim. \& Michelang.

Chaetogastra pentamera (Ule) P.J.F. Guim. \& Michelang.

Chaetogastra pleromoides (Naudin) P.J.F. Guim. \& Michelang.

Chaetogastra pulcherrima (Gleason) P.J.F. Guim. \& Michelang.

Chaetogastra purpurascens (Cogn.) P.J.F. Guim. \& Michelang. 
Chaetogastra purpusii (Brandegee) P.J.F. Guim. \& Michelang.

Chaetogastra repens (Wurdack) P.J.F. Guim. \& Michelang.

Chaetogastra rhynchantherifolia (Cogn.) P.J.F. Guim. \& Michelang.

Chaetogastra riograndensis F.S. Mey.

Chaetogastra rojasii (Cogn.) P.J.F. Guim. \& Michelang.

Chaetogastra roseotincta (Todzia) P.J.F. Guim. \& Michelang.

Chaetogastra rufipilis (Schltdl.) Walp.

Chaetogastra rupestris (Cogn.) P.J.F. Guim. \& Michelang.

Chaetogastra rusbyi (Cogn.) P.J.F. Guim. \& Michelang.

Chaetogastra sandiensis (Wurdack) P.J.F. Guim. \& Michelang.

Chaetogastra saxicola (F.S.Mey., P.J.F.Guim. \& R.Goldenb.) P.J.F. Guim. \& Michelang.

Chaetogastra saxosa (Gleason) P.J.F. Guim. \& Michelang.

Chaetogastra scabriuscula (Schlecht.) P.J.F. Guim. \& Michelang.

Chaetogastra schiedeana (Schltdl. \& Cham.) Walp.

Chaetogastra sebastianapolitana (Raddi) P.J.F. Guim. \& Michelang.

Chaetogastra sericea (de Santiago) P.J.F. Guim. \& Michelang.

Chaetogastra simplicicaulis (Naudin) P.J.F. Guim. \& Michelang.

Chaetogastra sinaloensis (Todzia) P.J.F. Guim. \& Michelang.

Chaetogastra solmsii (Cogn.) P.J.F. Guim. \& Michelang.

Chaetogastra stenopetala (Triana) P.J.F. Guim. \& Michelang.

Chaetogastra stenophylla (Cogn.) P.J.F. Guim. \& Michelang.

Chaetogastra tetrapetala (Cogn.) P.J.F. Guim. \& Michelang.

Chaetogastra thulia (Todzia) P.J.F. Guim. \& Michelang.

Chaetogastra tortuosa (Bonpl.) DC.

Chaetogastra triflora (Gleason) P. J. F. Guim. \& Michelang.

Chaetogastra trinitensis (Cogn.) P.J.F. Guim. \& Michelang.

Chaetogastra urbanii (Cogn.) P.J.F. Guim. \& Michelang.

Chaetogastra venosa (Gleason) P.J.F. Guim. \& Michelang.

Chaetogastra versicolor (Lindl.) P.J.F. Guim. \& Michelang.

Chaetogastra violacea (Cogn.) P.J.F. Guim. \& Michelang.

Chaetogastra wasshausenii (Wurdack) P.J.F. Guim. \& Michelang.

Chaetogastra weberbaueri (Cogn.) P.J.F. Guim. \& Michelang.

Chaetolepis alpina Naudin

Chaetolepis anisandra Naudin

Chaetolepis cufodontisii Standl.

Chaetolepis lindeniana (Naudin) Triana

Chaetolepis loricarella Triana

Chaetolepis microphylla (Bonpl.) Miq.

Chaetolepis perijensis Wurdack

Chaetolepis phelpsiae Gleason

Chaetolepis santamartensis Wurdack

Chaetolepis sessilis Pittier

Chaetostoma albiflorum (Naudin) Koschnitzke \& A.B. Martins

Chaetostoma armatum (Spreng.) Cogn.

Chaetostoma cupressinum (D. Don) Koschnitzke \& A.B. Martins

Chaetostoma fastigiatum Naudin

Chaetostoma flavum Koschnitzke \& A.B. Martins

Chaetostoma glaziovii Cogn.

Chaetostoma hexapetalum D. Nunes, D.O. Diniz, Koschnitzke \& M.J. Silva

Chaetostoma inerme Naudin

Chaetostoma riedelianum Cogn.

Chaetostoma scoparia Cogn.

Chaetostoma selagineum (Naudin) Koschnitzke \& A.B. Martins

Chaetostoma stenocladon (Naudin) Koschnitzke \& A.B. Martins

Chalybea brevipedunculata Penneys, C. Ulloa \& D. Fernández

Chalybea calyptrata (Penneys \& M.E. Morales) Penneys \& M.E. Morales 
Chalybea corymbifera Naudin

Chalybea ecuadorensis (Wurdack) Penneys \& M.E. Morales

Chalybea kirkbridei (Wurdack) M.E. Morales \& Penneys

Chalybea macrocarpa (L. Uribe) M.E. Morales \& Penneys

Chalybea minor (L. Uribe) M.E. Morales \& Penneys

Chalybea mutisiana (L. Uribe) M.E. Morales \& Penneys

Chalybea occidentalis (Lozano \& N. Ruiz-R.) M.E. Morales \& Penneys

Chalybea penduliflora (Wurdack) M.E. Morales \& Penneys

Chalybea peruviana M.E. Morales \& Penneys

Clidemia ferox Gleason

Clidemia minutiflora (Triana) Cogn.

Comolia anomala Pittier

Comolia berberifolia (Humb. \& Bonpl.) DC.

Comolia bracteosa Huber

Comolia latifolia (Aubl.) Cogn.

Comolia leptophylla (Bonpl.) Naudin

Comolia microphylla Benth.

Comolia nummularioides (Bonpl.) Naudin

Comolia ovalifolia (DC.) Triana

Comolia prostrata Wurdack

Comolia serpyllacea Wurdack

Comolia sessilis (Spreng.) Triana

Comolia villosa (Aubl.) Triana

Comoliopsis coriacea (Gleason) M.J.R.Rocha \& P.J.F.Guim.

Comoliopsis montana (Gleason) M.J.R.Rocha \& P.J.F.Guim.

Comoliopsis neblinae Wurdack

Desmoscelis calcarata (Naudin) Triana

Desmoscelis villosa (Aubl.) Naudin

Dicrananthera hedyotoideae C.Presl.

Diolena boliviensis Cogn.

Eriocnema acaulis (Cham.) Triana

Ernestia adenotricha L. Uribe

Ernestia blackii Brade \& Markgr.

Ernestia cataractae Tutin

Ernestia confertiflora Wurdack

Ernestia goudotii Triana

Ernestia granvillei Wurdack

Ernestia karuruana Wurdack

Ernestia lata Gleason

Ernestia maguirei Wurdack

Ernestia minor Gleason

Ernestia ovata Cogn.

Ernestia quadriseta O. Berg ex Triana

Ernestia rubra Pulle

Ernestia tenella (Bonpl.) DC.

Fritzschia anisostemon Cham.

Fritzschia atropurpurea D.Nunes, M.J.R.Rocha \& P.J.F.Guim.

Fritzschia cordifolia R.Romero, D.Nunes \& M.J.R.Rocha

Fritzschia edmundoi (Brade) M.J.R.Rocha \& P.J.F.Guim.

Fritzschia erecta Cham.

Fritzschia furnensis R. Romero \& M. J. Rocha

Fritzschia integrifolia Cham.

Fritzschia lanceiflora (DC.) M.J.R.Rocha \& P.J.F.Guim.

Fritzschia rupestris R. Pacifico, Almeda \& D. Nunes

Fritzschia sertularia (DC.) M.J.R.Rocha \& P.J.F.Guim.

Fritzschia sessilis (Spreng.) M.J.R.Rocha \& P.J.F.Guim. 
Fritzschia stenodon (Triana) M.J.R.Rocha \& P.J.F.Guim.

Graffenrieda anomala Triana

Graffenrieda barahonensis Urb.

Graffenrieda bella Almeda

Graffenrieda boliviensis Cogn.

Graffenrieda calyptrelloides Wurdack

Graffenrieda caryophyllea Triana

Graffenrieda caudata Wurdack

Graffenrieda chrysandra (Griseb.) Triana

Graffenrieda cinna J.F. Macbr.

Graffenrieda cinnoides Gleason

Graffenrieda colombiana Gleason

Graffenrieda conostegioides Triana

Graffenrieda cucullata (Triana) L.O. Williams

Graffenrieda emarginata (Ruiz \& Pav.) Triana

Graffenrieda fantastica R.E. Schult. \& L.B. Sm.

Graffenrieda foliosa Cogn.

Graffenrieda fruticosa Wurdack

Graffenrieda galeottii (Naudin) L.O. Williams

Graffenrieda gentlei Lundell

Graffenrieda glandulosa R. Goldenb. \& Meirelles

Graffenrieda goldenbergii L.F. Lima, Baumgratz, Nic Lugh. \& J.U. Santos

Graffenrieda gracilis (Triana) L.O. Williams

Graffenrieda grandifolia Gleason

Graffenrieda harlingii Wurdack

Graffenrieda hitchcockii Gleason

Graffenrieda intermedia Triana

Graffenrieda irwinii Wurdack

Graffenrieda jauana Wurdack

Graffenrieda jefensis Almeda, Alvear \& H. Mend.

Graffenrieda kralii Wurdack

Graffenrieda laevicarpa Michelang. \& R. Goldenb.

Graffenrieda lanceolata Gleason

Graffenrieda latifolia (Naudin) Triana

Graffenrieda laurina Triana

Graffenrieda limbata Triana

Graffenrieda maklenkensis H. Mend., Alvear \& Almeda

Graffenrieda maturaca L.F. Lima, Baumgratz, Nic Lugh. \& J.U. Santos

Graffenrieda meridensis Wurdack

Graffenrieda miconioides Naudin

Graffenrieda micrantha (Gleason) L.O. Williams

Graffenrieda moaensis Wurdack

Graffenrieda moritziana Triana

Graffenrieda obliqua Triana

Graffenrieda ottoschulzii (Urb. \& Ekman) Urb. \& Ekman

Graffenrieda patens Triana

Graffenrieda pedunculata Gleason

Graffenrieda penneysii Michelang. \& C. Ulloa

Graffenrieda phoenicea Markgr.

Graffenrieda polymera Gleason

Graffenrieda reticulata Wurdack

Graffenrieda robusta (Cogn.) L.O. Williams

Graffenrieda rotundifolia (Bonpl.) DC.

Graffenrieda rufa Wurdack

Graffenrieda rufescens Britton \& P. Wilson

Graffenrieda rupestris Ducke 
Graffenrieda santamartensis Wurdack

Graffenrieda scandens (Gleason) Wurdack

Graffenrieda sessilifolia Triana

Graffenrieda sipapoana Wurdack

Graffenrieda steyermarkii Wurdack

Graffenrieda tamana Wurdack

Graffenrieda tricalcarata Gleason

Graffenrieda trichanthera Gleason

Graffenrieda tristis (Triana) L.O. Williams

Graffenrieda uribei Wurdack

Graffenrieda versicolor Gleason

Graffenrieda weddellii Naudin

Henriettea acunae (Alain) Alain

Henriettea aggregata (D. Don) J.F. Macbr.

Henriettea angustifolia O. Berg ex Triana

Henriettea barkeri (Urb. \& Ekman) Alain

Henriettea boliviensis (Cogn.) Penneys, Michelang., Judd \& Almeda

Henriettea bracteosa (Wurdack) Penneys, Michelang., Judd \& Almeda

Henriettea caudata (Gleason) Penneys, Michelang., Judd \& Almeda

Henriettea ciliata (Urb. \& Ekman) Alain

Henriettea cuabae (Urb.) Borhidi

Henriettea cuneata (Standl.) L.O. Williams

Henriettea duckeana (Hoehne) Penneys, Michelang., Judd \& Almeda

Henriettea ekmanii (Urb.) Alain

Henriettea fascicularis (Sw.) M. Gómez

Henriettea fissanthera (Gleason) Penneys, Michelang., Judd \& Almeda

Henriettea flavescens (Aubl.) Baill.

Henriettea gibberosa (Urb.) Alain

Henriettea glabra (Vell.) Penneys, Michelang., Judd \& Almeda

Henriettea gomesii Brade

Henriettea goudotiana (Naudin) Penneys, Michelang., Judd \& Almeda

Henriettea granulata $\mathrm{O}$. Berg \& Triana

Henriettea heteroneura (Gleason) Penneys, Michelang., Judd \& Almeda

Henriettea hondurensis (Wurdack) Penneys, Michelang., Judd \& Almeda

Henriettea horridula Pilg.

Henriettea hotteana (Urb. \& Ekman) Alain

Henriettea ininiensis (Wurdack) Penneys, Michelang., Judd \& Almeda

Henriettea lasiostylis Pilg.

Henriettea lateriflora (Vahl) R.A. Howard \& E.A. Kellogg

Henriettea lawrancei (Gleason) Penneys, Michelang., Judd \& Almeda

Henriettea loretensis (Gleason) J.F. Macbr.

Henriettea lundellii (Wurdack) Penneys, Michelang., Judd \& Almeda

Henriettea macfadyenii (Triana) Alain

Henriettea maguirei (Wurdack) Penneys, Michelang., Judd \& Almeda

Henriettea manarae (Wurdack) Penneys, Michelangeli, Judd \& Almeda

Henriettea maroniensis Sagot

Henriettea martiusii (DC.) Naudin

Henriettea megaloclada (Urb. \& Ekman) Alain

Henriettea membranifolia (Cogn.) Alain

Henriettea mucronata (Gleason) S.S. Renner

Henriettea multiflora Naudin

Henriettea multigemma Carmenate \& Michelang.

Henriettea odorata (Markgr.) Penneys, Michelang., Judd \& Almeda

Henriettea ovata (Cogn.) Penneys, Michelang., Judd \& Almeda

Henriettea patrisiana DC.

Henriettea prancei (Wurdack) Penneys, Michelang., Judd \& Almeda 
Henriettea punctata M. Gomez

Henriettea ramiflora (Sw.) DC.

Henriettea reflexa (Urb. \& Ekman) Alain

Henriettea rimosa (Wurdack) Penneys, Michelang., Judd \& Almeda

Henriettea saldanhaei Cogn.

Henriettea seemannii (Naudin) L.O. Williams

Henriettea sessilifolia (L.) Alain

Henriettea sierrae Carmenate \& Bécquer

Henriettea spruceana Cogn.

Henriettea squamata (Alain) Alain

Henriettea squamulosa (Cogn.) Judd

Henriettea stellaris O. Berg ex Triana

Henriettea steyermarkii (Wurdack) Penneys, Michelang., Judd \& Almeda

Henriettea strigosa Gleason

Henriettea succosa (Aubl.) DC.

Henriettea sylvestris (Gleason) J.F. Macbr.

Henriettea tachirensis (Wurdack) Penneys, Michelang., Judd \& Almeda

Henriettea tobagensis (Wurdack) Penneys, Michelang., Judd \& Almeda

Henriettea tovarensis (Cogn.) Penneys, Michelang., Judd \& Almeda

Henriettea trachyphylla (Triana) Penneys, Michelang., Judd \& Almeda

Henriettea triflora (Vahl) Alain

Henriettea tuberculosa (Donn. Sm.) L.O. Williams

Henriettea uniflora Judd, Skean \& Penneys

Henriettea verrucosa (Triana) J.F. Macbr.

Henriettea williamii Brade

Henriettea williamsii (Pittier) Penneys, Michelangeli, Judd \& Almeda

Heterocentron alatum Rose \& Standl.

Heterocentron breedlovei (Wurdack) P.J.F. Guim. \& Michelang.

Heterocentron chiapense (Wurdack) P.J.F. Guim. \& Michelang.

Heterocentron chimalapanum Todzia

Heterocentron elegans (Schltdl.) Kuntze

Heterocentron evansii Almeda

Heterocentron glandulosum Schenk

Heterocentron hirtellum (Cogn.) L.O. Williams

Heterocentron laxiflorum Standl.

Heterocentron mexicanum Hook. \& Arn.

Heterocentron muricatum Gleason

Heterocentron purpureum S. Winkl.

Heterocentron subtriplinervium (Link \& Otto) A. Braun \& C.D. Bouché

Heterocentron suffruticosum Brandegee

Huberia bradeana Bochorny \& R.Goldenb.

Huberia capixaba (R.Goldenb. \& Reginato) Bochorny \& Michelang.

Huberia capixaba (R.Goldenb. \& Reginato) Bochorny \& Michelang.

Huberia carvalhoi Baumgratz

Huberia cogniauxii Baumgratz

Huberia consimilis Baumgratz

Huberia cordifolia (Cogn.) Bochorny \& R.Goldenb.

Huberia corymbosa (Cogn.) Bochorny \& R.Goldenb.

Huberia edmundoi (Brade) Bochorny \& R.Goldenb.

Huberia espiritosantensis Baumgratz

Huberia glazioviana Cogn.

Huberia glutinosa (Cogn.) Bochorny \& R.Goldenb.

Huberia hirsuta Bochorny \& R.Goldenb.

Huberia huberioides (Brade) Bochorny \& R.Goldenb.

Huberia insignis (Cham.) Bochorny \& R.Goldenb.

Huberia kollmannii (R.Goldenb. \& R.Tav.) Bochorny \& Michelang. 
Huberia laurina DC.

Huberia limae (Brade) Bochorny \& R. Goldenb.

Huberia lumiarensis (Bochorny, Michelang. \& R.Goldenb.) Bochorny

Huberia magdalenensis (Brade) Bochorny \& R.Goldenb.

Huberia mestrealvarensis (D.T.Iglesias \& R.Goldenb.) Bochorny \& Michelang.

Huberia minor Cogn.

Huberia minutifolia Bochorny \& R.Goldenb.

Huberia misteriosa Bochorny \&. R. Goldenb.

Huberia mourae (Cogn.) Bochorny \& R. Goldenb.

Huberia nettoana Brade

Huberia organensis (Saldanha \& Cogn.) Bochorny \& R.Goldenb.

Huberia ovalifolia DC.

Huberia parvifolia Cogn.

Huberia peruviana Cogn.

Huberia piranii Baumgratz

Huberia semiserrata DC.

Huberia sessilifolia R. Goldenb. \& Michelang.

Huberia souzalimae (Brade) Bochorny \& R.Goldenb.

Huberia staminodia Baumgratz

Huberia triplinervis Cogn.

Huberia weberbaueriana Baumgratz

Kirkbridea pentamera Wurdack

Kirkbridea tetramera Wurdack

Lavoisiera adamantium Pedersoli

Lavoisiera alba DC.

Lavoisiera angustifolia Cogn.

Lavoisiera arachnoidea Almeda \& A.B. Martins

Lavoisiera belinelloi A.B. Martins \& Almeda

Lavoisiera bradeana Barreto

Lavoisiera canastrensis Almeda \& A.B. Martins

Lavoisiera caryophyllea Naudin

Lavoisiera chamaepitys Naudin

Lavoisiera cougniaxiana Barreto

Lavoisiera confertiflora Naudin

Lavoisiera cordata Cogn. ex Glaz.

Lavoisiera crassifolia DC.

Lavoisiera daviesiana Almeda \& A.B. Martins

Lavoisiera firmula DC.

Lavoisiera gentianoides DC.

Lavoisiera glandulifera Naudin

Lavoisiera grandiflora Naudin

Lavoisiera harleyi Wurdack

Lavoisiera humilis Naudin

Lavoisiera imbricata DC.

Lavoisiera itambana DC.

Lavoisiera macrocarpa Naudin

Lavoisiera mello-barretoi Markgr.

Lavoisiera minima A.B. Martins \& Almeda

Lavoisiera mucorifera DC.

Lavoisiera nervulosa Naudin

Lavoisiera pohliana O. Berg ex Arens

Lavoisiera pulchella Cham.

Lavoisiera pulcherrima DC.

Lavoisiera punctata DC.

Lavoisiera quinquenervis Wurdack

Lavoisiera rigida Cogn. 
Lavoisiera rundeliana Almeda \& A.B. Martins

Lavoisiera sampaioana Barreto

Lavoisiera scaberula Naudin

Lavoisiera senaei Schwacke

Lavoisiera setosa A.B. Martins \& Almeda

Lavoisiera subulata Triana

Lavoisiera tetragona DC.

Lavoisiera vestita Almeda \& A.B. Martins

Leiostegia vernicosa Benth.

Lithobium cordatum Bong.

Loricalepis atlantica Amorim, Michelang. \& R.Goldenb.

Loricalepis duckei Brade

Macairea axilliflora Wurdack

Macairea cardonae Wurdack

Macairea chimantensis Wurdack

Macairea cuieirasii S.S. Renner

Macairea duidae Gleason

Macairea lanata Gleason

Macairea lasiophylla (Benth.) Wurdack

Macairea linearis Gleason

Macairea maroana Wurdack

Macairea multinervia Benth.

Macairea neblinae Wurdack

Macairea pachyphylla Benth.

Macairea parvifolia Benth.

Macairea philipsonii Renner

Macairea radula (Bonpl.) DC.

Macairea rigida Benth.

Macairea rufescens DC.

Macairea spruceana O. Berg ex Triana

Macairea stylosa Triana

Macairea sulcata Triana

Macairea theresiae Cogn.

Macairea thyrsiflora DC.

Macrocentrum andinum Michelang. \& R. Goldenb.

Macrocentrum anfractum Wurdack

Macrocentrum angustifolium Gleason

Macrocentrum anychioides Gleason

Macrocentrum brevipedicellatum Wurdack

Macrocentrum chimantense Wurdack

Macrocentrum cristatum (DC.) Triana

Macrocentrum droseroides Triana

Macrocentrum fasciculatum (DC.) Triana

Macrocentrum fruticosum Gleason

Macrocentrum gesneriaceum Sandwith

Macrocentrum gracile Wurdack

Macrocentrum huberi Wurdack

Macrocentrum latifolium Wurdack

Macrocentrum longidens (Gleason) Wurdack

Macrocentrum maguirei Wurdack

Macrocentrum minus Gleason

Macrocentrum neblinae Wurdack

Macrocentrum parvulum Gleason

Macrocentrum repens (Gleason) Wurdack

Macrocentrum rubescens Gleason

Macrocentrum steyermarkii Wurdack 
Macrocentrum stipulaceum Wurdack

Macrocentrum vestitum Sandwith

Macrocentrum yaracuyense Wurdack

Maguireanthus ayangannae Wurdack

Mallophyton chimantense Wurdack

Marcetia acerosa DC.

Marcetia alba Ule

Marcetia bahiana (Ule) A.B. Martins

Marcetia bahiensis (Brade \& Markgr.) Wurdack

Marcetia bracteolaris Cogn.

Marcetia candolleana A.K.A. Santos \& A.B. Martins

Marcetia canescens Naudin

Marcetia cardosoana A.K.A. Santos \& A.B. Martins

Marcetia eimeariana A.B. Martins \& Woodgyer

Marcetia ericoides (Spreng.) Cogn.

Marcetia formosa Wurdack

Marcetia grandiflora Markgr.

Marcetia harleyi Wurdack

Marcetia hatschbachii A.B. Martins

Marcetia lanuginosa Wurdack

Marcetia latifolia Naudin

Marcetia luetzelburgii Markgr.

Marcetia lychnophoroides A.B. Martins

Marcetia macrophylla Wurdack

Marcetia mucugensis Wurdack

Marcetia nervulosa Markgr.

Marcetia nummularia Markgr.

Marcetia oxycoccoides Wurdack \& A.B. Martins

Marcetia paganuccii A.K.A. Santos \& A.B. Martins

Marcetia semiriana A.B. Martins

Marcetia shepherdii A.B. Martins

Marcetia sincorensis Wurdack

Marcetia taxifolia (A. St.-Hil.) DC.

Marcetia velutina Markgr.

Marcetia viscida Wurdack

Meriania acida (Markgr.) Wurdack

Meriania acostae Wurdack

Meriania aguaditensis H. Mend. \& Fern. Alonso

Meriania albertiae Wurdack

Meriania albiflora Carmenate \& Michelang.

Meriania almedae Wurdack

Meriania amischophylla Wurdack

Meriania ampla Wurdack

Meriania amplexicaulis Wurdack

Meriania angustifolia (Cogn.) Carmenate \& Michelang.

Meriania antioquiensis L. Uribe

Meriania aracaensis Wurdack

Meriania arborea (Naudin) Triana

Meriania ardyae D. Fernández \& Dellinger

Meriania arizae H. Mend. \& Fern. Alonso

Meriania aurata C. Ulloa, D. Fernández \& D.A. Neill

Meriania axinioides Gleason

Meriania barbosae H. Mend., Alvear \& Almeda

Meriania baumgratziana R. Goldenb. \& Michelang.

Meriania boliviensis Cogn.

Meriania brachycera (Naudin) H. Mend. \& Fern. Alonso 
Meriania brevipedunculata Judd \& Skean

Meriania brittoniana Wurdack

Meriania broccha Wurdack

Meriania calophylla (Cham.) Triana

Meriania calyptrata (Naudin) Triana

Meriania campii Wurdack

Meriania claussenii (Naudin) Triana

Meriania colombiana Gleason

Meriania compressicaulis Almeda \& Penneys

Meriania cordifolia (H. Karst.) Triana

Meriania costata Wurdack

Meriania crassiramis (Naudin) Wurdack

Meriania cuneifolia Gleason

Meriania cuzcoana Wurdack

Meriania denticulata (Gleason) Wurdack

Meriania dimorphanthera Wurdack

Meriania drakei (Cogn.) Wurdack

Meriania ekmanii Urb.

Meriania excelsa (Gardner) Cogn.

Meriania fantastica Alvear, H. Mend. \& Almeda

Meriania finicola Wurdack

Meriania franciscana C. Ulloa \& Homeier

Meriania furvanthera Wurdack

Meriania glabra (DC.) Triana

Meriania glazioviana Cogn.

Meriania grandiflora (Standl.) Almeda

Meriania haemantha (Planch. \& Lindl.) H. Mend. \& Fern. Alonso

Meriania heptamera Lozano \& Alvear

Meriania hernandi L. Uribe

Meriania hexamera Sprague

Meriania horrida C. Ulloa \& Achá

Meriania hoyosii (Wurdack) Almeda \& Penneys

Meriania huilensis Wurdack

Meriania inflata Michelang. \& R. Goldenb.

Meriania involucrata (Desr.) Naudin

Meriania kirkbridei Wurdack

Meriania leucantha (Sw.) Sw.

Meriania longipes O. Berg ex Triana

Meriania loxensis Gleason

Meriania macrophylla (Benth.) Triana

Meriania maguirei Wurdack

Meriania maxima Markgr.

Meriania mexiae Wurdack

Meriania microflora Rob.Fernandez, R.Goldenb. \& Michelang.

Meriania mutabilis (Gleason) H. Mend. \& Fern. Alonso

Meriania mutisii (Bonpl.) H. Mend. \& Fern. Alonso

Meriania neblinensis Wurdack

Meriania ninakurorum (Bussmann \& Paniagua) E. Cotton \& Balslev

Meriania nobilis Triana

Meriania odorata Almeda

Meriania ornata Wurdack

Meriania pallida Gleason

Meriania panamensis Gleason

Meriania paniculata (DC.) Triana

Meriania parvifolia Judd \& Skean

Meriania pastazana Wurdack 
Meriania peltata L. Uribe

Meriania phlomoides (Triana) Almeda

Meriania pichinchensis Wurdack

Meriania prunifolia D. Don

Meriania pulcherrima Herzog

Meriania purpurea (Sw.) Sw.

Meriania quintuplinervis Naudin

Meriania radula (Benth.) Triana

Meriania rigida (Benth.) Triana

Meriania robusta Cogn.

Meriania rubriflora Michelang. \& R. Goldenb.

Meriania rugosa Markgr.

Meriania sanchezii R. Goldenb.

Meriania sanguinea Wurdack

Meriania sararensis H. Mend. \& Fern. Alonso

Meriania sclerophylla Triana

Meriania selvaflorensis H. Mend.

Meriania silverstonei H. Mend. \& Fern. Alonso

Meriania speciosa (Bonpl.) Naudin

Meriania splendens Triana

Meriania squamulosa Urb. \& Ekman

Meriania stellata (Gleason) Wurdack

Meriania steyermarkii Gleason

Meriania subumbellata Cogn.

Meriania tetragona (Cogn.) Wurdack

Meriania tetramera Wurdack

Meriania tetraquetra Triana

Meriania tomentosa (Cogn.) Wurdack

Meriania urceolata Triana

Meriania vargasii Wurdack

Meriania versicolor L. Uribe

Meriania vilcabambensis Wurdack

Meriania weberbaueri J.F. Macbr.

Meriania yalconensis H. Mend. \& Fern. Alonso

Meriania zunacensis D. Fernández \& Dellinger

Merianthera bullata R. Goldenb., Fraga \& A.P. Fontana

Merianthera burlemarxii Wurdack

Merianthera eburnea R. Goldenb. \& Fraga

Merianthera parvifolia R. Goldenb., Fraga \& A.P. Fontana

Merianthera pulchra Kuhlm.

Merianthera sipolisii (Glaz. \& Cogn.) Wurdack

Merianthera verrucosa R. Goldenb., Fraga \& A.P. Fontana

Miconia abbottii (Urb.) Ionta \& Judd

Miconia abbreviata Markgr.

Miconia abeggii Urb. \& Ekman

Miconia ablusa (Wurdack) Michelang.

Miconia abscondita Majure, Judd \& Skean

Miconia abysmophila Wurdack

Miconia acalephoides Naudin

Miconia acanthocoryne Wurdack

Miconia acinodendron (L.) Sweet

Miconia acostae (Wurdack) Michelang.

Miconia acreana Ule

Miconia acuminata (Steud.) Naudin

Miconia acuminifera Triana

Miconia acuminoides Skean, Judd \& Majure 
Miconia acunae Borhidi

Miconia acunagalei Judd, Bécquer \& Majure

Miconia acurensis (Wurdack) Michelang.

Miconia acutiflora (Naudin) R. Goldenb.

Miconia acutifolia Ule

Miconia acutilamina Michelang.

Miconia acutipetala Sprague

Miconia adamantinensis (Brade) R. Goldenb.

Miconia adenocalyx Urb. \& Ekman

Miconia adenothrix (Cogn.) R. Goldenb.

Miconia adinantha Wurdack

Miconia adrieni J.F. Macbr.

Miconia adventitia Michelang.

Miconia aenigmatica Wurdack

Miconia aequatorialis Wurdack

Miconia aeruginosa Naudin

Miconia affinis DC.

Miconia aggregata Gleason

Miconia agrestis (Aubl.) Baill.

Miconia aguaclarensis (Wurdack) Michelang.

Miconia aguilarii (Kriebel \& Almeda) Gamba \& Almeda

Miconia aguirrei L. Uribe

Miconia aguitensis Gleason

Miconia alainii Judd \& Skean

Miconia alata (Aubl.) DC.

Miconia alaticaulis Michelang.

Miconia alatiflora Michelang.

Miconia alatissima Gamba \& Almeda

Miconia albertii Gleason

Miconia albertobrenesii Gamba \& Almeda

Miconia albertsmithii Michelang.

Miconia albicans (Sw.) Steud.

Miconia albiviridis Urb. \& Ekman

Miconia alboglandulosa Gamba \& Almeda

Miconia alborosea L. Uribe

Miconia alborufescens Naudin

Miconia aligera Wurdack

Miconia aliquantula Wurdack

Miconia allardii (Wurdack) Michelang.

Miconia allenii (Almeda) Almeda

Miconia alloeotricha (Urb.) Judd, Penneys \& Skean

Miconia almedae (Kriebel) Michelang.

Miconia alpestris Cogn.

Miconia alpina Cogn.

Miconia alternans Naudin

Miconia alternidomatia Michelang.

Miconia alternifolia (Griseb.) Alain

Miconia alternilamina Michelang.

Miconia alterninervia (Cogn.) R. Goldenb.

Miconia altissima Cogn.

Miconia altomacaensis (Baumgratz \& D'El Rei Souza) R. Goldenb.

Miconia alypifolia Naudin

Miconia amabilis Cogn.

Miconia amacurensis Wurdack

Miconia amambayensis Kraenzl.

Miconia amapaensis R. Goldenb. \& Hinoshita 
Miconia amazonica Triana

Miconia amblyandra Naudin

Miconia amilcariana Almeda \& Dorr

Miconia amissa Wurdack

Miconia amnicola Wurdack

Miconia amoena Triana

Miconia amorimii (Reginato \& R. Goldenb.) R. Goldenb.

Miconia ampla Triana

Miconia amplexicaulis Naudin

Miconia amplilamina Michelang.

Miconia amplinodis Umaña \& Almeda

Miconia amygdaloides (DC.) R. Goldenb.

Miconia anaectocalyx Michelang.

Miconia anchicayensis Gamba \& Almeda

Miconia ancistrophora (C. Wright) Triana

Miconia andersonii Fawc. \& Rendle

Miconia andreana Cogn.

Miconia androsaemifolia Griseb.

Miconia angelana R. Romero \& R. Goldenb.

Miconia angulata (Griseb.) M. Gomez

Miconia angustidentata Almeda \& Penneys

Miconia angustifolia (Sw.) Griseb.

Miconia angustilamina (Judd \& Skean) Judd \& Ionta

Miconia anisophylla Triana

Miconia anisotricha (Schltdl.) Triana

Miconia annulata (Naudin) Triana

Miconia anoriensis (L. Uribe) Michelang.

Miconia antillana Skean, Judd \& Majure

Miconia antioquiensis Wurdack

Miconia aphanantha (Naudin) Michelang.

Miconia apiculata Urb. \& Ekman

Miconia apleura (Urb. \& Ekman) Judd \& Bécquer

Miconia aplostachya (Bonpl.) DC.

Miconia aponeura Triana

Miconia appendiculata Triana

Miconia approximata Gamba \& Almeda

Miconia aprica Gleason

Miconia aptera (DC.) R. Goldenb.

Miconia araguensis Wurdack

Miconia araneifera (Markgr.) Michelang. \& R. Goldenb.

Miconia arboricola Almeda

Miconia arbutifolia Naudin

Miconia archeri Wurdack

Miconia argentea (Sw.) DC.

Miconia argentimuricata Majure \& Judd

Miconia argyraea Cogn.

Miconia argyrophylla DC.

Miconia aristata Gleason

Miconia aristigera (Naudin) R. Goldenb. \& Michelang.

Miconia ascendens Wurdack

Miconia ascenditricha Judd, Bécquer \& Majure

Miconia asclepiadea Triana

Miconia aspera (Cogn.) R. Goldenb.

Miconia aspergillaris (Bonpl.) Naudin

Miconia asperifolia (Naudin) Majure \& Judd

Miconia asperiuscula (DC.) R. Goldenb. 
Miconia asperrima Triana

Miconia asplundii Wurdack

Miconia aspratilis Wurdack

Miconia astrocalyx Meirelles \& R. Goldenb.

Miconia astroplocama Donn. Sm.

Miconia astrotricha (DC.) Triana

Miconia asymmetrica Michelang.

Miconia atlantica Caddah \& R. Goldenb.

Miconia atrata (Spreng.) Wawra

Miconia atrofusca Cogn.

Miconia atropilis Cogn. \& Gleason

Miconia atropurpurea Gamba \& Almeda

Miconia atroviridis (Cogn.) R. Goldenb.

Miconia attenuatifolia Michelang.

Miconia augustgrisebachii Ionta \& Judd

Miconia augusti Cogn.

Miconia augustkappleri Michelang.

Miconia aulocalyx Mart. ex Triana

Miconia aurantiaca (Almeda \& Kriebel) Gamba \& Almeda

Miconia aurantipetala Michelang.

Miconia aurea (D. Don) Naudin

Miconia aureoides Cogn.

Miconia auricoma (Spring ex Mart.) R. Goldenb.

Miconia aurifolia Bécquer \& Judd

Miconia auritinoda Wurdack

Miconia australis (Cham.) R. Goldenb.

Miconia avia Wurdack

Miconia axillaris (Macfad.) Skean, Judd \& Majure

Miconia axinaeoides Gleason

Miconia ayacuchensis Wurdack

Miconia ayangannensis (Wurdack) Michelang.

Miconia ayisyena Skean, Judd \& Majure

Miconia aymardii Wurdack

Miconia badilloi Michelang.

Miconia bahiana R.Goldenb. \& J.Coelho

Miconia bahorucensis Skean, Judd \& Majure

Miconia bailloniana J.F. Macbr.

Miconia baillonii M. Gómez

Miconia bairdiana (Skean, Judd, Clase \& Peguero) Skean \& Judd

Miconia balansae (Cogn.) R. Goldenb.

Miconia balbisiana (Ser. ex DC.) Michelang.

Miconia bangii Cogn.

Miconia baracoana M. Gómez

Miconia baracoensis Urb.

Miconia barahonensis (Urb. \& Ekman) Ionta \& Judd

Miconia barbata (Borhidi) Judd, Bécquer \& Majure

Miconia barbeyana Cogn.

Miconia barbinervis (Benth.) Triana

Miconia barbipetiolata Michelang.

Miconia barbipilis Gleason

Miconia barclayana Wurdack

Miconia barkeri Urb. \& Ekman

Miconia barkleyi (Wurdack) Michelang.

Miconia basilensis Urb. \& Ekman

Miconia baumgratziana R. Goldenb. \& C.V. Martin

Miconia bella Wurdack 
Miconia beneolens Wurdack

Miconia benoistii Wurdack

Miconia bensparrei Gamba \& Almeda

Miconia benthamiana Triana

Miconia benthamii (Naudin) Michelang.

Miconia bernardii Wurdack

Miconia berteroi (DC.) Judd \& Ionta

Miconia biacuta Cogn.

Miconia biappendiculata (Naudin) L. Uribe

Miconia bicolor (Mill.) Triana

Miconia biflora (Cogn.) Judd

Miconia biformis Cogn.

Miconia bigibbosa (Cogn.) Michelang.

Miconia biglandulosa Gleason

Miconia biglomerata (Bonpl.) DC.

Miconia bilopezii Wurdack

Miconia biolleyana (Cogn.) Gamba \& Almeda

Miconia bipatrialis Wurdack

Miconia biperulifera Cogn.

Miconia birimosa (Naudin) Skean, Judd \& Majure

Miconia bisecundiflora Ocampo \& Almeda

Miconia biserrata (DC.) Michelang.

Miconia bissei (Bécquer) Bécquer \& Michelang.

Miconia bisulcata Urb.

Miconia blakeifolia Gleason

Miconia blancheana Urb.

Miconia blepharodes (DC.) R. Goldenb.

Miconia boekei (Wurdack) Gamba \& Almeda

Miconia bolivarensis Wurdack

Miconia boliviensis Cogn.

Miconia boomii Wurdack

Miconia bordoncilloana Lozano \& M.E. Morales

Miconia borhidiana Judd, Bécquer \& Majure

Miconia borinquensis Skean, Judd \& Majure

Miconia borjensis Wurdack

Miconia brachyanthera Triana

Miconia brachybotrya Triana

Miconia brachycalyx Triana

Miconia brachycentra (Griseb.) M. Gómez

Miconia brachygyna Gleason

Miconia brachyloba (Urb.) Bécquer

Miconia brachystemon (Urb.) Judd, Bécquer \& Majure

Miconia brackenridgei (A. Gray) R. Goldenb.

Miconia bracteata (DC.) Triana

Miconia bracteolata (Bonpl.) DC.

Miconia bracteosa (Naudin) Michelang.

Miconia bractiflora Gamba \& Almeda

Miconia bradeana Baumgratz \& R. Goldenb.

Miconia brasiliensis Triana

Miconia brenesii Standl.

Miconia breteleri Wurdack

Miconia breviflora (Cogn.) R. Goldenb.

Miconia brevifolia (Urb. \& Ekman) Skean \& Judd

Miconia brevipes Benth.

Miconia brevis J.F. Macbr.

Miconia brevistylis Cogn. 
Miconia brevitheca Gleason

Miconia brigitteae H. Mend. \& Posada-Herrera

Miconia brittonii Cogn.

Miconia broadwayi (Urb.) Michelang.

Miconia brunescens (Urb.) Bécquer \& Skean

Miconia brunnea Mart. ex DC.

Miconia bubalina (D. Don) Naudin

Miconia bucherae Alain

Miconia buddlejoides Triana

Miconia bullata (Turcz.) Triana

Miconia bullatifolia Michelang.

Miconia bullifera (Mart. \& Schrank ex DC.) Michelang.

Miconia bullosa (DC.) Michelang.

Miconia bullotricha Bécquer \& Majure

Miconia buntingii Wurdack

Miconia burchellii Triana

Miconia buxifolia Naudin

Miconia cabraliensis (Wurdack) R. Goldenb.

Miconia cabucu Hoehne

Miconia cacatin (Aubl.) S.S. Renner

Miconia cacumina Wurdack

Miconia caelata (Bonpl.) DC.

Miconia caelestis (Standl.) Michelang.

Miconia caerulea (Pav. ex D. Don) Naudin

Miconia caesariata Wurdack

Miconia caesia Cogn. \& Gleason

Miconia caiuia E.C.O. Chagas \& R. Goldenb.

Miconia cajalbanensis Judd, Bécquer \& Majure

Miconia cajanumana Wurdack

Miconia calignosa Wurdack

Miconia calocoma Almeda

Miconia calvescens DC.

Miconia calycina Cogn.

Miconia calycopteris (Rich.) Judd, Bécquer \& Majure

Miconia campanensis Urb. \& Ekman

Miconia campestris (Benth.) Triana

Miconia campii Wurdack

Miconia camporum (Brade) R. Goldenb.

Miconia canaguensis Wurdack

Miconia canaimana Michelang.

Miconia cancellata (Cogn.) R. Goldenb.

Miconia candelabrum (J.F. Macbr.) R. Goldenb.

Miconia cannabina Markgr.

Miconia canoi Michelang. \& D.F. Paredes

Miconia capillaris (Sw.) M. Gomez

Miconia capilliflora (Naudin) R. Goldenb.

Miconia capillinervis Ionta \& Judd

Miconia capillipes (Triana) Michelang.

Miconia capitellata Cogn.

Miconia capixaba R. Goldenb.

Miconia caquetana (Sprague) Michelang.

Miconia caquetensis (Gleason) Ocampo \& Almeda

Miconia carassana Cogn.

Miconia cardenasiae Jan. M. Burke \& Michelang.

Miconia carnea Cogn.

Miconia carolensis (Gleason) Michelang. 
Miconia carpishana Wurdack

Miconia caryophyllaea (DC.) Triana

Miconia castaneiflora Naudin

Miconia castillensis Wurdack

Miconia castrensis Wurdack

Miconia cataractae Triana

Miconia catharinensis (Cogn.) R. Goldenb.

Miconia caucana Gleason

Miconia caudata (Bonpl.) DC.

Miconia caudatifolia Michelang.

Miconia caudiculata Pittier

Miconia caudigera DC.

Miconia cauingia J.F. Macbr.

Miconia cautis Wurdack

Miconia cava Michelang. \& R. Goldenb.

Miconia cazaletii Wurdack

Miconia celaquensis Almeda

Miconia centrodesma Naudin

Miconia centrodesmoides Wurdack

Miconia centronioides Gleason

Miconia centrophora Naudin

Miconia centrosperma Almeda

Miconia cephaloides Michelang.

Miconia ceramicarpa (DC.) Cogn.

Miconia cerasiflora Urb.

Miconia cercophora Wurdack

Miconia cernua Naudin

Miconia cernuiflora Jan. M. Burke \& Michelang.

Miconia chaetocalyx (A. Gray) R. Goldenb.

Miconia chaetodon Naudin

Miconia chaetodonta R. Goldenb. \& Michelang.

Miconia chamissois Naudin

Miconia chapensis E. Cotton \& W. Meier

Miconia charadrophila (Tutin) Michelang.

Miconia charleswrightii Bécquer \& Judd

Miconia chartacea Triana

Miconia chemillensis L.A. Cárdenas

Miconia chichirivichensis Michelang.

Miconia chimantensis (Wurdack) Michelang.

Miconia chinantlana (Naudin) Almeda

Miconia chionophila Naudin

Miconia chiriquiensis Almeda

Miconia chlorocarpa Cogn.

Miconia chocoensis (Wurdack) Gamba \& Almeda

Miconia choriophylla Wurdack

Miconia christophoriana (Ham.) DC

Miconia chrysocoma Gleason

Miconia chrysoneura Triana

Miconia chrysophylla (Rich.) Urb.

Miconia ciliaris Triana

Miconia ciliata (Rich.) DC.

Miconia ciliolata (Cogn.) R. Goldenb.

Miconia cinchonifolia DC.

Miconia cineana Majure, Judd, Ionta \& Skean

Miconia cinerascens Miq.

Miconia cinerea Cogn. 
Miconia cinereiformis Ionta, Judd \& Skean

Miconia cinnabarina Jan. M. Burke \& Michelang.

Miconia cinnamomea Beurl.

Miconia cinnamomifolia (DC.) Naudin

Miconia cionotricha L. Uribe

Miconia cipoensis R. Goldenb.

Miconia circumscissa (Cogn.) R. Goldenb.

Miconia cladonia Gleason

Miconia clandestina (Almeda) Almeda

Miconia clasei Majure, Skean \& Judd

Miconia clathrantha Triana ex Cogn.

Miconia cleefii L. Uribe

Miconia clementiana (Britton) Bécquer \& Majure

Miconia clementii (P. Wilson) Bécquer

Miconia clivorum Wurdack

Miconia clypeata Wurdack

Miconia coadunata (Wurdack) R. Goldenb.

Miconia coarctiflora (Wurdack) Ocampo \& Almeda

Miconia coccinea (Rich.) Judd \& Skean

Miconia cocoensis Almeda \& Kriebel

Miconia codonostigma Gleason \& Wurdack

Miconia coelestis (Pav. ex D. Don) Naudin

Miconia cogniauxii (Glaz. ex D'El Rei Souza) R. Goldenb.

Miconia collatata Wurdack

Miconia collayensis Wurdack

Miconia colliculosa Almeda

Miconia coloradensis Almeda

Miconia commutata Almeda

Miconia comosa Cogn.

Miconia complanata Jan. M. Burke, Michelang. \& D. Fernández

Miconia compressa Naudin

Miconia compressicaulis Wurdack

Miconia comptifolia Wurdack

Miconia concinna Almeda

Miconia condylata Wurdack

Miconia confertiflora Almeda

Miconia conformis Wurdack

Miconia conglomerata (DC.) Michelang.

Miconia conica Skean, Judd \& Majure

Miconia coniophora Urb. \& Ekman

Miconia conoapiculata Michelang.

Miconia conoattenuata Michelang.

Miconia conobracteata Michelang.

Miconia conobrenesii Michelang.

Miconia conocentronioides Michelang.

Miconia conochiriquensis Michelang.

Miconia conocuatrecasii Michelang.

Miconia conodentata Michelang.

Miconia conohirtella Michelang.

Miconia conolancifolia Michelang.

Miconia conomacrantha Michelang.

Miconia conomicrantha Michelang.

Miconia conoplumosa Michelang.

Miconia conorubiginosa Michelang.

Miconia conorufescens Michelang.

Miconia conosetifera Michelang. 
Miconia conosetosa Michelang.

Miconia conospeciosa Michelang.

Miconia contrerasii Wurdack

Miconia cookii Gleason

Miconia cooperi (Cogn.) Michelang.

Miconia corallina Spring

Miconia corazonica Wurdack

Miconia corcovadensis (Raddi) R. Goldenb.

Miconia cordata Triana

Miconia cordifolia Wurdack

Miconia cordigera (Triana) R. Goldenb.

Miconia cordilamina Judd \& Bécquer

Miconia coriacea (Sw.) DC.

Miconia cornifera Humberto Mend., Posada-Herrera \& Heriberto David

Miconia cornifolia (Desr.) Naudin

Miconia cornoides (Schltdl. \& Cham.) Almeda

Miconia cornuta (Lozano \& N. Ruiz-R.) Almeda \& Alvear

Miconia coronata (Bonpl.) DC.

Miconia coronifera Wurdack

Miconia correae Almeda

Miconia corymbiformis Cogn.

Miconia corymbosa (Rich.) Judd \& Skean

Miconia cosangensis Wurdack

Miconia costaricensis Cogn.

Miconia costata (Urb.) Judd, Bécquer \& Majure

Miconia costoides (Michelang.) Michelang.

Miconia cottoniana Michelang.

Miconia cowanii Wurdack

Miconia crassifolia Triana

Miconia crassinervia Cogn.

Miconia crassinervis (Urb.) Skean, Judd \& Majure

Miconia crassipes Triana

Miconia crassistigma Cogn.

Miconia crebribullata Wurdack

Miconia cremadena Gleason

Miconia cremersii (Wurdack) Ocampo \& Almeda

Miconia cremophylla Naudin

Miconia crenata (Vahl) Michelang.

Miconia crenulata (Gleason) Michelang.

Miconia cretacea Gleason

Miconia crinita Naudin

Miconia cristalensis (Borhidi) Judd, Bécquer \& Majure

Miconia cristata (Reginato \& R. Goldenb.) R. Goldenb.

Miconia croatii (Almeda) Michelang. \& Almeda

Miconia crocata Almeda

Miconia crocea (Desr.) Naudin

Miconia crocina Michelang.

Miconia crossosepala (Griseb.) Michelang.

Miconia crotonifolia (Desr.) Judd \& Ionta

Miconia cruenta Triana

Miconia cuatrecasasii Markgr.

Miconia cubacinerea Majure \& Judd

Miconia cubana (Alain) Majure \& Judd

Miconia cubatanensis Hoehne

Miconia cubensis (C. Wright ex Griseb.) C. Wright

Miconia cucullata (Gleason) Michelang. 
Miconia cundinamarcensis Wurdack

Miconia cuneata Triana ex Cogn.

Miconia cuneatissima R. Goldenb. \& Michelang.

Miconia cupeyalensis Bécquer \& Michelang.

Miconia cuprea Wurdack

Miconia cursoris (Wurdack) Michelang.

Miconia curta (Gleason) Wurdack

Miconia curvipila (Urb. \& Ekman) Ionta, Judd \& Skean

Miconia cuspidata Mart. ex Naudin

Miconia cuspidatissima Pittier

Miconia cutucuensis Wurdack

Miconia cyanocarpa Naudin

Miconia cyathanthera Triana

Miconia cymifera (Donn. Sm.) Michelang.

Miconia daironii Michelang.

Miconia danielii Almeda

Miconia dapsiliflora Wurdack

Miconia dasyclada Wurdack

Miconia dasytricha (A. Gray) R. Goldenb.

Miconia davidsei (Almeda) Almeda

Miconia debilis (Crueg.) Michelang.

Miconia decipiens Cogn.

Miconia decorticans (Bécquer) Bécquer \& Majure

Miconia decurrens Cogn.

Miconia deflexa (O. Berg ex Triana) R. Goldenb.

Miconia delicatula A. Rich.

Miconia demissifolia Wurdack

Miconia dendroides (Naudin) R. Goldenb.

Miconia densifolia Cogn.

Miconia dentata (D. Don) Michelang.

Miconia denticulata Naudin

Miconia dependens (Pav. ex D. Don) Judd \& Majure

Miconia desiliens (Gleason) Michelang.

Miconia desmantha Benth.

Miconia desportesii Urb.

Miconia diaphanea Gleason

Miconia dichroa Cogn.

Miconia dichrophylla J.F. Macbr.

Miconia diegogomezii Kriebel \& Almeda

Miconia dielsiana Urb.

Miconia dielsii Markgr.

Miconia difficilis Triana

Miconia diffusa (Cogn.) R. Goldenb.

Miconia diguensis (Wurdack) Michelang.

Miconia dimorphica (J.F. Macbr.) Michelang.

Miconia dimorphotheca Jan. M. Burke \& Michelang.

Miconia dioica Wurdack

Miconia dipsacea Naudin

Miconia discoidea (Lozano \& N. Ruiz-R.) Almeda \& Alvear

Miconia discolor DC.

Miconia discolorata Bécquer \& Michelang.

Miconia dispar Benth.

Miconia dissimulans Wurdack

Miconia dissita Almeda

Miconia dissitiflora Almeda

Miconia dissitinervia Kriebel, Almeda \& A. Estrada 
Miconia disticha Michelang.

Miconia divaricata Gardner

Miconia divaricatiflora Judd \& Bécquer

Miconia divergens Triana

Miconia divisoriana Wurdack

Miconia dodecandra Cogn.

Miconia dodsonii Wurdack

Miconia dolichantha (Naudin) R. Goldenb.

Miconia dolichodons (Cogn.) R. Goldenb.

Miconia dolichopoda Naudin

Miconia dolichorrhyncha Naudin

Miconia dolichostachya (Naudin) R. Goldenb.

Miconia domatiata (Urb. \& Ekman) Skean \& Judd

Miconia domingensis Cogn.

Miconia dominicensis (Penneys \& Judd) Penneys

Miconia domociliata Michelang.

Miconia doniana Naudin

Miconia dorsaliporosa R. Goldenb. \& Reginato

Miconia dorsiloba Gleason

Miconia drosera (Sagot) Michelang.

Miconia dubia (DC.) R. Goldenb.

Miconia duckei Cogn.

Miconia dudleyi Wurdack

Miconia duidae (Gleason) Michelang.

Miconia dulcis Jan. M. Burke \& Michelang.

Miconia dumetosa Cogn.

Miconia dunstervillei Wurdack

Miconia dura Triana

Miconia durandii Michelang. \& P.Jørg

Miconia dusenii (Cogn.) R. Goldenb.

Miconia ebracteata (Triana) R. Goldenb.

Miconia echinata (Griseb.) Judd, Bécquer \& Majure

Miconia echinocarpa Judd, Bécquer \& Majure

Miconia ecuadorensis (Gleason) Michelang.

Miconia edentula (Gleason) Michelang.

Miconia egensis Cogn.

Miconia egregia Wurdack

Miconia eichleri Cogn.

Miconia ekmanii (Urb.) Judd, Bécquer \& Majure

Miconia elaeodendron (DC.) Naudin

Miconia elaeoides Naudin

Miconia elata (Sw.) DC.

Miconia eleagnoides (Sw.) Griseb.

Miconia elegans Cogn.

Miconia elegantissima Bécquer \& Judd

Miconia ellipsoidea (Urb. \& Ekman) Ionta, Judd \& Skean

Miconia ellsworthii Michelang.

Miconia elongata Cogn.

Miconia elvirae Wurdack

Miconia emendata Wurdack

Miconia epibaterium (DC.) Michelang.

Miconia eremita L. Uribe

Miconia erikasplundii Gamba \& Almeda

Miconia erikmaniana Skean \& Judd

Miconia eriocalyx Cogn.

Miconia erioclada Triana 
Miconia eriodonta DC.

Miconia ernstii Wurdack

Miconia ernstulei Michelang.

Miconia erosa Gleason

Miconia erostrata (DC.) R. Goldenb.

Miconia erythrantha Naudin

Miconia erythropogon (DC.) Judd \& Ionta

Miconia espinosae Markgr.

Miconia eugenioides Triana

Miconia euphorbioides (Naudin) R. Goldenb.

Miconia evanescens (Almeda) Gamba \& Almeda

Miconia expansa Gleason

Miconia explicita Wurdack

Miconia extinctoria (Bonpl.) Michelang.

Miconia fabianiana Mabb.

Miconia fadyenii (Hook.) Judd \& Skean

Miconia falcata Cogn.

Miconia fallacissima (Markgr.) R. Goldenb.

Miconia fallax DC.

Miconia fanshawei Wurdack

Miconia farfanii Jan. M. Burke \& Michelang.

Miconia farinasii (Wurdack) Michelang.

Miconia fasciculata Gardner

Miconia fastigiata (Cogn.) R. Goldenb.

Miconia fausta (Wurdack) Michelang.

Miconia favosa (Desr.) Naudin

Miconia fenestrata (Benth.) Michelang.

Miconia ferreyrae Wurdack

Miconia ferruginata DC.

Miconia ferruginea (Desr.) DC.

Miconia fictilis (J.F. Macbr.) Michelang. \& R. Goldenb.

Miconia filamentosa Gleason

Miconia filiformis (Gleason) Michelang.

Miconia filisepala (Urb.) Judd, Bécquer \& Majure

Miconia firma J.F. Macbr.

Miconia fissa Gleason

Miconia fissinervia (Gleason) Michelang.

Miconia flaccida Gleason

Miconia flammea Casar.

Miconia flavescens Cogn.

Miconia flexuosa (Triana) Michelang.

Miconia floccosa Cogn.

Miconia florbella Schnell ex Michelang. \& R.Goldenb.

Miconia floribunda (Bonpl.) DC.

Miconia fluminensis Ule

Miconia foliosa Triana

Miconia folsomii (Almeda) Almeda

Miconia fontanae (Reginato \& R. Goldenb.) R. Goldenb.

Miconia foreroi (Wurdack) Michelang.

Miconia formicaria Gamba \& Almeda

Miconia formicocollina Michelang.

Miconia formicofoliosa Michelang.

Miconia formicoheterophylla Michelang.

Miconia formicojuruensis Michelang.

Miconia formicosetosa Michelang.

Miconia formonense (Judd, Skean \& Clase) Judd, Bécquer \& Majure 
Miconia formosa Cogn.

Miconia forzzae R. Goldenb. \& Hinoshita

Miconia fosbergii Wurdack

Miconia fosteri Wurdack

Miconia foveolata Cogn.

Miconia fragilis Naudin

Miconia fragrans Cogn.

Miconia fragrantissima (Almeda) Michelang. \& Almeda

Miconia francavillana Cogn.

Miconia fraterna (Gleason) Michelang.

Miconia freyreissii (Cogn.) R. Goldenb.

Miconia friedmaniorum Almeda \& Umaña

Miconia friedrichlehmannii R. Goldenb.

Miconia frontinoana Cogn. \& Gleason

Miconia fruticulosa Cogn.

Miconia fuertesii Cogn.

Miconia fuliginosa Wurdack

Miconia fulvostellata L.O. Williams

Miconia funckii Wurdack

Miconia furfuracea (Vahl) Griseb.

Miconia galactantha Naudin

Miconia galdamesiae Kriebel \& Almeda

Miconia galeiformis Jan. M. Burke \& Michelang.

Miconia galeottii (Naudin) Michelang.

Miconia garcia-barrigae (Wurdack) Almeda

Miconia gentryi Wurdack

Miconia georgebuntingii Michelang.

Miconia gibba Markgr.

Miconia gigantea Cogn.

Miconia gigantophylla (Britton) Michelang.

Miconia gilva Cogn.

Miconia glaberrima (Schltdl.) Naudin

Miconia glabrata Cogn.

Miconia glabrifolia Judd, Bécquer \& Majure

Miconia glandulifera Cogn.

Miconia glanduliflora R. Goldenb. \& Michelang.

Miconia glandulipetala Ocampo \& Almeda

Miconia glandulistyla Wurdack

Miconia glandulosa (Sw.) Naudin

Miconia glaucescens Triana

Miconia glazioviana Cogn.

Miconia gleasoniana Wurdack

Miconia globulifera Naudin

Miconia globuliflora (Rich.) Cogn.

Miconia glomerata Triana

Miconia glomerulifera Cogn.

Miconia glomeruliflora Judd, Bécquer \& Majure

Miconia gloriosa (Macfad.) Michelang.

Miconia glutinosa Cogn.

Miconia glyptophylla Wurdack

Miconia goldenbergiana Caddah

Miconia gonioclada Triana

Miconia goniostigma Triana

Miconia gonoptera (Gleason) Michelang.

Miconia gorzulae (Wurdack) Michelang. \& R. Goldenb.

Miconia gossypina Triana 
Miconia goudotii Naudin

Miconia graciliflora (Huber) Michelang.

Miconia gracilifolia Ionta \& Judd

Miconia gracilipes (Gleason) Michelang.

Miconia gracilis Triana

Miconia granatensis (Gleason) Ocampo \& Almeda

Miconia grandibracteata Judd, Bécquer \& Majure

Miconia grandidentata Almeda

Miconia grandiflora Cogn.

Miconia grandifoliata R. Goldenb. \& Michelang.

Miconia granulata (Urb.) Majure \& Judd

Miconia granvillei (Wurdack) Michelang.

Miconia gratissima Benth. ex Triana

Miconia grayana Cogn.

Miconia grayumii Almeda

Miconia grenadensis (Penneys \& Judd) Penneys

Miconia griffisii J.F. Macbr.

Miconia grisea Cogn.

Miconia grisebachiana Bécquer \& Michelang.

Miconia grossidentata Wurdack

Miconia guadalupensis (DC.) Judd, Ionta, \& Majure

Miconia guaiquinimae Wurdack

Miconia guajaibonensis Judd, Bécquer \& Majure

Miconia guatemalensis Cogn.

Miconia guayaquilensis (Bonpl.) D. Don ex DC.

Miconia gynoverrucosa (Reginato) R. Goldenb.

Miconia hadrophylla Wurdack

Miconia haemantha (Triana ex Cogn.) Skean, Judd \& Majure

Miconia haemanthoides Skean, Judd \& Majure

Miconia hamata Cogn.

Miconia hammelii (Almeda) Almeda

Miconia hanstuerckheimii Michelang.

Miconia harlingii Wurdack

Miconia hatschbachii (Brade) R. Goldenb.

Miconia haughtii (Gleason) Wurdack

Miconia heliotropoides Triana

Miconia hematostemon Naudin

Miconia hemenostigma Naudin

Miconia henripittieri (Kriebel) Michelang.

Miconia heptamera (Wurdack) Michelang.

Miconia herincquiana (Cogn.) R. Goldenb.

Miconia hermogenesii (Baumgratz \& D'El Rei Souza) R. Goldenb.

Miconia hernandogarciae H. Mend. \& Idárraga

Miconia herpetica DC.

Miconia herrerae Gleason

Miconia herzogii Cogn.

Miconia heterochaeta Wurdack

Miconia heteroclita (Naudin) Michelang.

Miconia heteromera Naudin

Miconia heteronervis (Naudin) Michelang.

Miconia heteroneura (Schrank \& Mart. ex DC.) Michelang.

Miconia heterophylla (Naudin) M. Gómez

Miconia heteroporata (Reginato) R. Goldenb.

Miconia heterothrix Gleason \& Wurdack

Miconia heterotricha Wurdack

Miconia hexamera Wurdack 
Miconia hexapetala Wurdack

Miconia hildeana Kriebel \& Almeda

Miconia hirsuta (Sw.) Judd, Bécquer \& Majure

Miconia hirsutivena Gleason

Miconia hirta Cogn.

Miconia hirtella Cogn.

Miconia hirtellicaulis Ionta \& Judd

Miconia hirticalyx Judd \& Bécquer

Miconia hirticaulis Skean, Judd \& Majure

Miconia hirtistyla Bécquer \& Majure

Miconia hispaniolica Judd \& Majure

Miconia hispidula (Cogn.) Judd, Bécquer \& Majure

Miconia histothrix Wurdack

Miconia holosericea (L.) DC.

Miconia hondurensis Donn. Sm.

Miconia hookeriana Triana

Miconia horrida (Cogn.) R. Goldenb.

Miconia hospitalis Wurdack

Miconia hotteana (Urb. \& Ekman) Judd \& Ionta

Miconia hotteana (Urb. \& Ekman) Judd \& Ionta

Miconia hottensis Ionta, Judd \& Skean

Miconia howardiana Judd, Salzman \& Skean

Miconia huanucensis Wurdack

Miconia huberi Wurdack

Miconia huigrensis Wurdack

Miconia humifusa Jan. M. Burke \& Michelang.

Miconia hutchisonii Wurdack

Miconia hybophylla (Urb.) Majure \& Judd

Miconia hyemalis A. St.-Hil. \& Naudin

Miconia hygrophila Naudin

Miconia hylophila Wurdack

Miconia hymenanthera Triana

Miconia hyperprasina Naudin

Miconia hypiodes Urb. \& Ekman

Miconia hypoglauca (C. Wright ex Griseb.) Judd, Bécquer \& Majure

Miconia hypoleuca (Benth.) Triana

Miconia ibaguensis (Bonpl.) Triana

Miconia ibarrae Almeda

Miconia icosandra Gleason

Miconia idiogena Wurdack

Miconia idroboi Wurdack

Miconia iluensis Wurdack

Miconia imbricata Gleason

Miconia imitans Wurdack

Miconia imparilis (Wurdack) Michelang.

Miconia impetiolaris (Sw.) D. Don ex DC.

Miconia impressa (Urb.) Judd, Bécquer \& Majure

Miconia impressinervis Skean \& Judd

Miconia inaequalifolia Triana

Miconia inaequalis R. Goldenb. \& Michelang.

Miconia inaequidens (DC.) Naudin

Miconia inaequipetiolata Majure \& Judd

Miconia inamoena Pilg.

Miconia inanis Cogn. \& Gleason ex Gleason

Miconia incachacana Wurdack

Miconia incerta (Wurdack) Gamba \& Almeda 
Miconia inconspicua Miq.

Miconia incurva Gleason

Miconia indicoviolacea Gamba, Almeda \& Alvear

Miconia ingens Wurdack

Miconia innata Gleason

Miconia inobsepta (Wurdack) Michelang.

Miconia inopinata (Almeda) Almeda

Miconia insueta Wurdack

Miconia insularis Gleason

Miconia integrifolia Cogn.

Miconia intermedia (DC.) Ocampo \& Almeda

Miconia intonsa (E. Cotton \& W. Meier) Michelang.

Miconia intricata Triana

Miconia inusitata (Wurdack) Michelang. \& R. Goldenb.

Miconia ioneura Griseb.

Miconia ionopogon (Mart.) R. Goldenb.

Miconia irwinii Wurdack

Miconia itatiaiae (Wawra) R. Goldenb.

Miconia iteophylla Almeda

Miconia jahnii Pittier

Miconia jaliscana (Standl.) Michelang.

Miconia jamesluteynii Michelang.

Miconia jashaferi Majure \& Judd

Miconia javorkaeana Borhidi

Miconia jefensis Almeda

Miconia jentaculorum Wurdack

Miconia jimenezii Judd \& R.S. Beaman

Miconia jitotolana Wurdack

Miconia jorgensenii Wurdack

Miconia josecuatrecasasii R. Goldenb. \& Michelang.

Miconia jucunda (DC.) Triana

Miconia juddii (Skean) Skean, Judd \& Majure

Miconia julianii Michelang.

Miconia juliansteyermarkii R. Goldenb. \& Michelang.

Miconia juruensis Pilg.

Miconia kappellei Almeda \& Kriebel

Miconia kappleri Naudin

Miconia karlkrugii Majure \& Judd

Miconia karsticola Judd, Bécquer, Skean \& Majure

Miconia kavanayensis Wurdack

Miconia klotzschii Triana

Miconia klugii Gleason

Miconia koepckeana Wurdack

Miconia kollmannii R. Goldenb. \& Reginato

Miconia kraenzlinii Cogn.

Miconia kriegeriana Baumgratz \& Chiaveg.

Miconia krugiana (Cogn.) Majure \& Judd

Miconia krugii Cogn.

Miconia kuntzei Cogn.

Miconia labiakiana Goldenb. \& C.V. Martin

Miconia lacera (Bonpl.) Naudin

Miconia lachnoclada Wurdack

Miconia laciniata Wurdack

Miconia lacunosa (Cogn.) R. Goldenb.

Miconia laeta Cogn.

Miconia laetivirens L. Uribe 
Miconia laevifolia (Gleason) Michelang.

Miconia laevigata (L.) D. Don

Miconia laevipilis Wurdack

Miconia lagunensis Ule

Miconia lambayequensis Wurdack

Miconia lamprarrhena Triana

Miconia lamprophylla Triana

Miconia lanata (DC.) Triana

Miconia lanatifolia Judd, Bécquer \& Majure

Miconia lanceifolia (Urb.) Ionta, Judd \& Skean

Miconia lanceolata (Desr.) DC.

Miconia lancifolia (Spruce ex Triana) Michelang.

Miconia lapae (D'El Rei Souza \& Baumgratz) R. Goldenb.

Miconia lappacea (DC.) Triana

Miconia larensis Gleason

Miconia lasiocalyx Cogn.

Miconia lasiopetala (Cogn.) Almeda

Miconia lasiopoda (Benth.) Michelang.

Miconia lasiostachya (Cogn.) R. Goldenb.

Miconia lasiostyla Gleason

Miconia lasseri Gleason

Miconia latecrenata (DC.) Naudin

Miconia lateriflora Cogn.

Miconia latidecurrens Gamba \& Almeda

Miconia latifolia (D. Don) Naudin

Miconia latistigma Cogn.

Miconia laurina (D. Don) Naudin

Miconia laxa Wurdack

Miconia laxiflora (Schltdl.) Michelang.

Miconia laxivenula (Wurdack) Gamba \& Almeda

Miconia leabiscoriacea R. Goldenb.

Miconia leabiswarmingiana R. Goldenb.

Miconia leablanchetiana R. Goldenb.

Miconia leacalvescens R. Goldenb.

Miconia leacapitata R. Goldenb.

Miconia leacinnamomifolia R. Goldenb.

Miconia leacollina R. Goldenb.

Miconia leaconfusa R. Goldenb.

Miconia leacongestiflora R. Goldenb.

Miconia leacordifolia R. Goldenb.

Miconia leacoriacea R. Goldenb.

Miconia leacrenata R. Goldenb. \& Michelang.

Miconia leacuminata R. Goldenb.

Miconia leacuneata R. Goldenb.

Miconia leadebilis R. Goldenb.

Miconia leadentata R. Goldenb.

Miconia leadispar R. Goldenb.

Miconia leaechinata R. Goldenb.

Miconia leaeichleri R. Goldenb.

Miconia leaeriocalyx R. Goldenb.

Miconia leafallax R. Goldenb.

Miconia leaflavescens R. Goldenb.

Miconia leafloribunda R. Goldenb.

Miconia leafluminensis R. Goldenb.

Miconia leafoveolata R. Goldenb.

Miconia leafragilis R. Goldenb. 
Miconia leagracilis R. Goldenb.

Miconia leagrayana R. Goldenb.

Miconia leahirtella R. Goldenb.

Miconia leahumilis R. Goldenb.

Miconia leakleinii R. Goldenb.

Miconia lealancifolia R. Goldenb.

Miconia lealindeniana Michelang. \& R. Goldenb.

Miconia lealpestris R. Goldenb.

Miconia leamarginata R. Goldenb.

Miconia leamarkgrafii R. Goldenb.

Miconia leamollis R. Goldenb.

Miconia leamourae R. Goldenb.

Miconia leandroides Cogn. \& Gleason ex Gleason

Miconia leaneurotricha R. Goldenb.

Miconia leaoblongifolia R. Goldenb.

Miconia leaorganensis R. Goldenb.

Miconia leaovata R. Goldenb.

Miconia leapallida R. Goldenb.

Miconia leapennipilis R. Goldenb.

Miconia leapolychaeta R. Goldenb.

Miconia learamboi R. Goldenb.

Miconia leariedeliana R. Goldenb.

Miconia learigida R. Goldenb.

Miconia leasaldanhae R. Goldenb.

Miconia leasanguinea R. Goldenb.

Miconia leasylvatica R. Goldenb.

Miconia leatetragona R. Goldenb.

Miconia leatetraptera R. Goldenb.

Miconia leathyrsiflora R. Goldenb.

Miconia leatomentosa R. Goldenb.

Miconia leatrata R. Goldenb.

Miconia leatristis R. Goldenb.

Miconia leatruncata R. Goldenb.

Miconia leaumbellata R. Goldenb.

Miconia leavelutina R. Goldenb.

Miconia leawarmingiana R. Goldenb.

Miconia leblondii Judd \& Skean

Miconia lechleri Triana

Miconia ledifolia (DC.) Naudin

Miconia lehmannii Cogn.

Miconia leiotricha Wurdack

Miconia lenticellata Alain

Miconia lepidota DC.

Miconia leptantha Urb. \& Ekman

Miconia leptopus (Triana) Michelang. \& R. Goldenb.

Miconia leticiana (Michelang.) Michelang.

Miconia leucandra (C. Wright ex Griseb.) Judd \& Ionta

Miconia leucocarpa DC.

Miconia licrophora Wurdack

Miconia liebmannii Cogn.

Miconia liesneri Wurdack

Miconia ligulata Almeda

Miconia ligustrina (Sm.) Triana

Miconia ligustroides (DC.) Naudin

Miconia lilacina Triana

Miconia lima (Desr.) M. Gómez 
Miconia limbata (Cogn.) R. Goldenb.

Miconia limitaris Wurdack

Miconia limoides (Urb.) Majure \& Judd

Miconia lindeniana (Naudin) M. Gómez

Miconia lindmanii (Urb.) Bécquer \& Michelang.

Miconia linearis (Gleason) Michelang.

Miconia linnaeoides (Hook. f.) Michelang.

Miconia liogieri Bécquer \& Michelang.

Miconia lithophila L. Uribe

Miconia littlei Wurdack

Miconia livida Triana

Miconia loligomorpha (R. Goldenb. \& Reginato) R. Goldenb.

Miconia lomensis (Urb.) Michelang.

Miconia lonchophylla Naudin

Miconia longibarbis (Schrank \& Mart. ex DC.) R. Goldenb.

Miconia longibracteata Almeda

Miconia longicollis (Urb. \& Cogn.) Judd \& Bécquer

Miconia longicoma (Cogn.) Ocampo \& Almeda

Miconia longicuspidata S.S. Renner \& R. Goldenb.

Miconia longicuspis Cogn.

Miconia longidentata Michelang. \& W. Meier

Miconia longifolia (Aubl.) DC.

Miconia longipetiolata (Brade) R. Goldenb.

Miconia longiracemosa Gleason

Miconia longirostrata R. Romero, Fontelas \& Versiane

Miconia longisepala Gleason

Miconia longisetosa Wurdack

Miconia longispicata Triana

Miconia lorenzouribei Michelang.

Miconia loretensis Pilg.

Miconia loreyoides Triana

Miconia lourteigiana Wurdack

Miconia loxensis (Bonpl.) DC.

Miconia lucenae R.Goldenb. \& Michelang.

Miconia luciana Gleason

Miconia lucianobernadii Michelang.

Miconia lucida Naudin

Miconia luctatoris (Wurdack) R. Goldenb.

Miconia lugonis Wurdack

Miconia lugubris Cogn.

Miconia lundelliana L.O. Williams

Miconia lundellii (Wurdack) Almeda

Miconia lurida Cogn.

Miconia lutea (Cogn.) R. Goldenb.

Miconia luteola Cogn.

Miconia lutescens (Bonpl.) DC.

Miconia luteynii Wurdack

Miconia lutgardae Bécquer \& Michelang.

Miconia lymanii Wurdack

Miconia macayana Judd \& Skean

Miconia macbrydeana Wurdack

Miconia machinazana C. Ulloa \& D.A. Neill

Miconia macrantha Triana

Miconia macrocarpa (Urb. \& Ekman) Judd \& Ionta

Miconia macrodon (Naudin) Wurdack

Miconia macropetala Michelang. 
Miconia macrophysca (Spruce ex Triana) Michelang.

Miconia macropora (Triana) R. Goldenb.

Miconia macroptera (Naudin) Michelang.

Miconia macrosperma (Mart.) Michelang.

Miconia macrothyrsa Benth.

Miconia macrotis Cogn.

Miconia maculata (Urb. \& Ekman) Judd, Bécquer \& Majure

Miconia macuxi Meirelles, Caddah \& R. Goldenb.

Miconia madisonii Wurdack

Miconia madrensis Standl.

Miconia magdalenae Triana

Miconia magdalenensis (Brade) R. Goldenb.

Miconia magnipetala (R. Goldenb. \& E. Camargo) R. Goldenb.

Miconia maguirei Gleason

Miconia mailyniae H. Mend. \& Aguirre-Santoro

Miconia majalis Cogn.

Miconia malatestae J.F. Macbr.

Miconia manarae (Wurdack) Michelang.

Miconia manauara R. Goldenb., Caddah \& Michelang.

Miconia mandonii Cogn.

Miconia manicata Cogn. \& Gleason

Miconia manserichensis (Wurdack) Michelang.

Miconia mansfeldiana Urb. \& Ekman

Miconia mantuensis (Britton \& P. Wilson) Bécquer

Miconia mapirensis Gleason

Miconia marahuacensis (Wurdack) Michelang.

Miconia marginata Triana

Miconia mariae Wurdack

Miconia marigotiana (Urb. \& Ekman) Majure \& Judd

Miconia maroana Wurdack

Miconia martiniana Gleason

Miconia martinicensis Cogn.

Miconia matthaei Naudin

Miconia mattogrossensis Hoehne

Miconia mattosii (Baumgratz \& D'El Rei Souza) R. Goldenb.

Miconia matudae (L.O. Williams) Michelang.

Miconia mavacana (Wurdack) Michelang.

Miconia maximowicziana Cogn.

Miconia mayarensis (Urb.) Bécquer \& Michelang.

Miconia mayeta (D. Don) Michelang.

Miconia mazanana J.F. Macbr.

Miconia mazatecana de Santiago

Miconia mcphersonii Almeda \& Penneys

Miconia mevaughii Wurdack

Miconia media (D. Don) Naudin

Miconia mediocris Wurdack

Miconia medusa Gleason

Miconia megalantha Gleason

Miconia melanodesma (Naudin) Michelang. \& R. Goldenb.

Miconia melanotricha (Triana) Gleason

Miconia melastomoides (Raddi) R. Goldenb.

Miconia melinonis Naudin

Miconia mellina DC.

Miconia membranacea Triana

Miconia mendoncaei Cogn.

Miconia meridensis Triana 
Miconia meridionalis (D'El Rei Souza) R. Goldenb.

Miconia mesmeana Gleason

Miconia mesoamericana Michelang.

Miconia metallica (Naudin) Triana

Miconia micayana Wurdack

Miconia michelangeliana R. Goldenb. \& L. Kollmann

Miconia miconiastrum (Naudin) R. Goldenb.

Miconia micrantha Cogn.

Miconia microdictya (Urb. \& Ekman) Skean, Judd \& Majure

Miconia microflora R. Goldenb. \& Michelang.

Miconia micropetala Cogn.

Miconia microphylla (C. Wright) M. Gómez

Miconia microphysca Michelang.

Miconia microstachya (Naudin) R. Goldenb.

Miconia microthyrsa (R.O. Williams) Michelang.

Miconia miguelfuertesii Ionta \& Judd

Miconia miles-morganii J.F. Macbr.

Miconia militis Wurdack

Miconia mimica Gleason

Miconia minuta Gleason

Miconia minutiflora (Bonpl.) DC.

Miconia miocarpa Naudin

Miconia mirabilis (Aubl.) L.O. Williams

Miconia mituana Wurdack

Miconia moana (Borhidi \& O. Muñiz) Bécquer \& Michelang.

Miconia mocquerysii Wurdack

Miconia modica J.F. Macbr.

Miconia moensis (Britton) Alain

Miconia molesta Cogn.

Miconia molinopampana Wurdack

Miconia mollicula Triana

Miconia mollis Triana

Miconia molybdea Naudin

Miconia monantha (L.O. Williams) Michelang.

Miconia monciona Urb. \& Ekman

Miconia monocephala Urb.

Miconia monopleura (Urb.) Bécquer \& Majure

Miconia monteleagreana (Cogn.) Michelang.

Miconia monzoniensis Cogn.

Miconia moorei Wurdack

Miconia morichensis (Wurdack) Michelang.

Miconia morii Almeda

Miconia mornicola A.C. Nicolson

Miconia mortoniana (Standl.) Michelang.

Miconia mourae Cogn.

Miconia mulleola Wurdack

Miconia multiflora Cogn. ex Britton

Miconia multiglandulosa Cogn.

Miconia multinervia Cogn.

Miconia multiplinervia Cogn.

Miconia multisetosa (Cogn.) R. Goldenb.

Miconia multispicata Naudin

Miconia munchicana (Lozano \& N. Ruiz-R.) Almeda \& Alvear

Miconia munizii (Borhidi) Ionta \& Bécquer

Miconia muricata (D. Don) Triana

Miconia muriculata (Almeda) Michelang. \& Almeda 
Miconia mutabilis (DC.) Triana

Miconia mutisiana Markgr.

Miconia myriantha Benth.

Miconia myrmecina (Gleason) Michelang.

Miconia myrmecodomatia Michelang.

Miconia myrtiformis Naudin

Miconia myrtillifolia Naudin

Miconia namandensis Wurdack

Miconia nambyquarae Hoehne

Miconia nanayensis (Wurdack) R. Goldenb. \& Michelang.

Miconia nanopetala R. Goldenb. \& Michelang.

Miconia nanophylla Judd, Bécquer \& Majure

Miconia napoana Wurdack

Miconia nasella Wurdack

Miconia navasensis (Britton \& P. Wilson) Ionta \& Bécquer

Miconia navicularis (Brade) R. Goldenb.

Miconia navifolia Ionta, Judd \& Skean

Miconia navioensis Wurdack

Miconia neblinae (Wurdack) Michelang.

Miconia neblinensis Wurdack

Miconia nebulensis Michelang.

Miconia neei Jan. M. Burke \& Michelang.

Miconia neglecta (Brade) R. Goldenb.

Miconia nematophora Urb. \& Ekman

Miconia neoamygdalina Skean, Judd \& Majure

Miconia neocalcarata Michelang.

Miconia neocoloradensis Almeda

Miconia neocordata Michelang.

Miconia neocoronata Gamba \& Almeda

Miconia neocrotonifolia Michelang.

Miconia neocrugeriana Michelang.

Miconia neoepiphytica Michelang.

Miconia neolanuginosa Almeda

Miconia neopectinata Almeda

Miconia neopilosa Michelang.

Miconia neosecunda Ocampo \& Almeda

Miconia neosecundiflora Ocampo \& Almeda

Miconia neourceolata Michelang.

Miconia neriifolia Triana

Miconia nervosa (Sm.) Triana

Miconia nervosissima Michelang. \& R. Goldenb.

Miconia neurotricha Cogn.

Miconia nianga (DC.) R. Goldenb.

Miconia niangaiformis (Cogn.) R. Goldenb.

Miconia niederleinii (Cogn.) R. Goldenb.

Miconia nigricans Cogn.

Miconia nigripes Cogn. \& Gleason

Miconia nipensis (Britton \& P. Wilson) Ionta \& Bécquer

Miconia nitens Benth.

Miconia nitida (D. Don) Naudin

Miconia nitidissima Cogn.

Miconia nobilis Gleason

Miconia nodosa Cogn.

Miconia norandina Michelang.

Miconia nordestina R. Goldenb. \& E.C.O. Chagas

Miconia norlindii (Urb.) Majure \& Judd 
Miconia notabilis Triana

Miconia novemnervia Naudin

Miconia nubicola Proctor

Miconia nutans Donn. Sm.

Miconia nystroemii Ekman ex Urb.

Miconia obconica Gleason \& Wurdack

Miconia obliqua Gleason

Miconia oblonga (Gleason) Michelang.

Miconia oblongifolia Cogn.

Miconia obovata Triana

Miconia obscura (Bonpl.) Naudin

Miconia obtusa (Griseb.) Triana

Miconia obtusifolia (Cogn.) Skean, Judd \& Majure

Miconia ocampensis Skean, Judd \& Majure

Miconia ochracea Triana

Miconia octona (Bonpl.) Judd \& Majure

Miconia octopetala Cogn.

Miconia odoratissima L.A. Cárdenas

Miconia oellgaardii E. Cotton

Miconia oerstediana (O. Berg ex Triana) Michelang.

Miconia oinochrophylla Donn. Sm.

Miconia oldemanii Wurdack

Miconia oleifolia (Griseb.) M. Gómez

Miconia oligantha Wurdack

Miconia oligocephala Donn. Sm.

Miconia oligochaeta (Cham.) R. Goldenb.

Miconia oligotricha (DC.) Naudin

Miconia ombrophila Wurdack

Miconia omissa Bécquer \& Michelang.

Miconia onaensis Wurdack

Miconia oocarpa (A. Gray) R. Goldenb.

Miconia opaca (Brade) R. Goldenb.

Miconia opacifolia J.F. Macbr.

Miconia oraria Wurdack

Miconia orcheotoma Naudin

Miconia oreogena Wurdack

Miconia orescia L. Uribe

Miconia organensis Gardner

Miconia ortiziae (Kriebel) Michelang.

Miconia osaensis Aguilar, Kriebel \& Almeda

Miconia ossaeifolia Urb. \& Ekman

Miconia ossaeiformis Naudin

Miconia ossaeoides (Naudin) Michelang. \& Tiernan

Miconia ostentata (Wurdack) Michelang.

Miconia ostrina (Gleason) Michelang.

Miconia ottikeri J.F. Macbr.

Miconia ottobuchtienii Michelang. \& R. Goldenb.

Miconia ottoschmidtii (Urb.) Majure \& Judd

Miconia ovalifolia Cogn.

Miconia ovata Cogn.

Miconia ovatifolia (Urb.) Judd, Bécquer \& Majure

Miconia oxyura (Naudin) Michelang.

Miconia pachyantha Bécquer

Miconia pachydonta Gleason

Miconia pachyphylla Cogn.

Miconia pachystachya (Wurdack) Michelang. 
Miconia paeminosa Wurdack

Miconia pagnolensis Majure \& Judd

Miconia pailasana Wurdack

Miconia pakaraimae (Wurdack) Michelang.

Miconia palcazuana Michelang. \& R. Goldenb.

Miconia paleacea Cogn.

Miconia paleaciramis Michelang. \& R. Goldenb.

Miconia palenquensis (Wurdack) Gamba \& Almeda

Miconia paludigena Wurdack

Miconia pandurata Triana

Miconia paniculata (Mart. \& Schrank ex DC.) Naudin

Miconia papillata (Cogn.) R. Goldenb.

Miconia papillopetala Kriebel \& Almeda

Miconia papillosa (Desr.) Naudin

Miconia papillosperma Michelang. \& R. Goldenb.

Miconia paradisica Wurdack

Miconia paradoxa (Mart. ex DC.) Triana

Miconia paraguayensis Cogn.

Miconia paralimoides Majure \& Judd

Miconia paralongicollis (Judd, Ionta, Clase \& Skean) Judd \& Bécquer

Miconia parasitica (O. Berg ex Triana) R. Goldenb.

Miconia parvifolia Cogn.

Miconia paspaloides Gleason

Miconia pastazana Wurdack

Miconia pastoensis Triana

Miconia paucartambensis Jan. M. Burke \& Michelang.

Miconia paucidens DC.

Miconia paulina (DC.) R. Goldenb.

Miconia paupercula (Naudin) Triana

Miconia pausana Wurdack

Miconia pavoniana Naudin

Miconia pectinata (Cogn.) R. Goldenb.

Miconia pedicellata Cogn.

Miconia pedunculata Majure \& Judd

Miconia peltata Almeda

Miconia peltifolia R. Goldenb. \& Michelang.

Miconia pendula Umaña \& Almeda

Miconia penduliflora Cogn.

Miconia penicillata Gleason

Miconia pennellii Gleason

Miconia penninervis (Griseb.) M. Gómez

Miconia penningtonii Wurdack

Miconia pentlandii Naudin

Miconia pepericarpa DC.

Miconia perclara (Wurdack) Michelang.

Miconia perelegans Urb.

Miconia perezii (Alain) Bécquer \& Michelang.

Miconia pergamentacea Cogn.

Miconia perijensis Wurdack

Miconia pernettifolia Triana

Miconia perobscura Wurdack

Miconia persicariifolia Cogn.

Miconia perturbata Wurdack

Miconia petersonii Urb.

Miconia petiolaris (Schltdl. \& Cham.) Michelang.

Miconia petiolata (DC.) Michelang. 
Miconia petroniana Cogn. \& Saldanha

Miconia petropolitana Cogn.

Miconia phaeochaeta Wurdack

Miconia phaeophylla Triana

Miconia phaeotricha Naudin

Miconia phanerostila Pilg.

Miconia phelpsiae (Gleason) R. Goldenb.

Miconia phlebodes Wurdack

Miconia phrynosomaderma Majure \& Judd

Miconia pichinchensis Benth.

Miconia picinguabensis R. Goldenb. \& A.B. Martins

Miconia pierreboissieriana R. Goldenb.

Miconia pilaloensis Wurdack

Miconia pileata DC.

Miconia pilgeriana Ule

Miconia pilifera (Urb.) Ionta \& Bécquer

Miconia pilonensis (Wurdack) R. Goldenb.

Miconia pilosa (Sw.) Judd \& Ionta

Miconia pilosissima (Cogn.) R. Goldenb.

Miconia piperifolia Triana

Miconia piperilamina Michelang.

Miconia pisinna Wurdack

Miconia pisinniflora Wurdack

Miconia pittieri Cogn.

Miconia planifilamentosa (Brade) R. Goldenb.

Miconia platyphylla (Benth.) L.O. Williams

Miconia platypoda Gleason

Miconia pleiocrassifolia R. Goldenb.

Miconia pleiofluminensis R. Goldenb. \& Michelang.

Miconia pleioglazioviana R. Goldenb.

Miconia pleiomagdalenensis R. Goldenb.

Miconia pleiomicrantha R. Goldenb.

Miconia pleioparvifolia R. Goldenb.

Miconia pleiorosea R. Goldenb.

Miconia pleiosetulosa R. Goldenb.

Miconia plena Gleason

Miconia plethorica Naudin

Miconia plicata (Griseb.) M. Gómez

Miconia plicatifolia Skean, Judd \& Majure

Miconia plukenetii Naudin

Miconia plumifera Triana

Miconia poecilantha L. Uribe

Miconia poeppigii Triana

Miconia poiretii (Griseb.) M. Gómez

Miconia polita Gleason

Miconia polyadena (Ule) R. Goldenb.

Miconia polyandra Gardner

Miconia polychaeta Wurdack

Miconia polychaete (Urb. \& Ekman) Ionta, Judd \& Skean

Miconia polyflora Skean, Judd \& Majure

Miconia polygama Cogn.

Miconia polyneura Triana

Miconia polystachya (Naudin) R. Goldenb.

Miconia polytopica Wurdack

Miconia poortmannii (Cogn.) Wurdack

Miconia popayanensis Wurdack 
Miconia porphyrotricha (Markgr.) Wurdack

Miconia portogallensis de Santiago \& Michelang.

Miconia portoricensis (Alain) Ionta, Judd \& Skean

Miconia pozoensis Wurdack

Miconia pozuzoana L.A. Cárdenas \& Michelang.

Miconia prancei Wurdack

Miconia prasina (Sw.) DC.

Miconia prasinifolia Gleason

Miconia pratensis Judd, Bécquer \& Majure

Miconia prietoi Wurdack

Miconia proctorii Judd

Miconia procumbens (Gleason) Wurdack

Miconia prominens Wurdack

Miconia protuberans Wurdack

Miconia pseudoalternifolia Michelang.

Miconia pseudoaplostachya Cogn.

Miconia pseudocapsularis Wurdack

Miconia pseudocentrophora Cogn.

Miconia pseudodebilis Michelang.

Miconia pseudoeichleri Cogn.

Miconia pseudofloribunda (Bécquer) Bécquer \& Michelang.

Miconia pseudopedicellata Judd \& Bécquer

Miconia pseudopinetorum (Borhidi \& O. Muñiz) Judd, Bécquer \& Majure

Miconia pseudoradula Cogn. \& Gleason

Miconia pseudorigida Proctor

Miconia psychrophila Naudin

Miconia pterocaulon Triana

Miconia pteroclada Urb.

Miconia pterosepala Urb.

Miconia puberula Cogn.

Miconia pubescens (Gleason) Michelang.

Miconia pubicalyx Gleason

Miconia pubipetala Miq.

Miconia pubistyla (Wurdack) R. Goldenb.

Miconia pujana Markgr.

Miconia pulchra Cogn.

Miconia pulgari J.F. Macbr.

Miconia pulverata Judd, Bécquer \& Majure

Miconia pulverulenta Ruiz \& Pav.

Miconia pulvinata Gleason

Miconia punctata (Desr.) D. Don ex DC.

Miconia punctibullata M.E. Morales, Michelang. \& F. González

Miconia punicea Wurdack

Miconia puracensis Wurdack

Miconia purpurea (D. Don) Judd \& Skean

Miconia purpureovillosa (Hoehne) R. Goldenb.

Miconia purpureoviolacea (Cogn.) Michelang.

Miconia purpuriflora Michelang. \& R. Goldenb.

Miconia purulensis Donn. Sm.

Miconia pusilliflora (DC.) Naudin

Miconia pustulata Naudin

Miconia pycnantha (Urb. \& Ekman) Ionta, Judd \& Skean

Miconia pycnaster (Tutin) Michelang.

Miconia pycnoneura Urb.

Miconia pyramidalis (Desr.) DC.

Miconia pyrifolia Naudin 
Miconia pyxidata (Proctor) Michelang.

Miconia quadrangularis (Sw.) Naudin

Miconia quadrialata S.S. Renner \& S. Beck

Miconia quadricaulis Michelang.

Miconia quadridomius Gamba \& Almeda

Miconia quadrifolia Naudin

Miconia quadripora Wurdack

Miconia quadrisulca (Naudin) Ocampo \& Almeda

Miconia quinquedentata (DC.) R. Goldenb.

Miconia quinquenervia (Mill.) Gamba \& Almeda

Miconia quinquenodis (DC.) R. Goldenb.

Miconia quintuplinervia Cogn.

Miconia rabenii Cogn.

Miconia racemifera (DC.) Triana

Miconia racemosa (Aubl.) DC.

Miconia raddii R. Goldenb.

Miconia radicans (Cogn.) Gamba \& Almeda

Miconia radula Cogn.

Miconia radulifolia (Benth.) Naudin

Miconia raggiana Michelang.

Miconia raimondiana (Markgr.) R. Goldenb. \& Michelang.

Miconia ramboi Brade

Miconia rangeliana C. Wright ex Griseb.

Miconia rariflora (Bonpl.) Michelang.

Miconia rava Wurdack

Miconia ravenii Wurdack

Miconia reburrosa Wurdack

Miconia reclinata (Bonpl.) Naudin

Miconia recondita Wurdack

Miconia reducens Triana

Miconia reflexa (Gleason) Michelang.

Miconia reflexipila Wurdack

Miconia refracta (Cogn.) R. Goldenb.

Miconia regelii Cogn.

Miconia reitziana (Cogn. \& Gleason) Gamba \& Almeda

Miconia reitzii (Wurdack) R. Goldenb.

Miconia remotiflora Urb.

Miconia renatogoldenbergii Meirelles \& Bacci

Miconia renatoi Gamba \& Almeda

Miconia rennerae E. Cotton

Miconia repens (Triana) Michelang.

Miconia reptans (R.Goldenb. \& Reginato) R. Goldenb.

Miconia resima Naudin

Miconia resimoides Cogn.

Miconia resinosa (Gleason) Michelang.

Miconia reticulata Triana

Miconia reticulato-venosa Judd, Bécquer \& Majure

Miconia retropila (DC.) Ocampo \& Almeda

Miconia retusa Pilg.

Miconia reversa (DC.) Ocampo \& Almeda

Miconia revolutifolia Skean, Judd \& Majure

Miconia rhamnifolia (Naudin) Ocampo \& Almeda

Miconia rheophytica Posada-Herrera \& Almeda

Miconia rhodantha Wurdack

Miconia rhodopetala (Donn. Sm.) Michelang.

Miconia rhodopogon (DC.) R. Goldenb. 
Miconia rhombifolia Alain

Miconia rhomboidea (Urb. \& Ekman) Skean \& Judd

Miconia rhytidophylla Naudin

Miconia ribesiiflora (Cham.) R. Goldenb.

Miconia ricardoi Kriebel \& Almeda

Miconia richardsprucei Michelang.

Miconia riedeliana (O.Berg ex Triana) R.Goldenb.

Miconia rigens Naudin

Miconia rigida (Sw.) Triana

Miconia rigidissima Urb. \& Ekman

Miconia rimachii Wurdack

Miconia rimalis Naudin

Miconia rimbachii Wurdack

Miconia riograndensis (Brade) R. Goldenb.

Miconia riparia Triana

Miconia rivalis Wurdack

Miconia rivetii Danguy \& Cherm.

Miconia robinsoniana Cogn.

Miconia robusta Cogn.

Miconia rodriguezii (Almeda) Almeda

Miconia rondoniensis Meirelles \& R. Goldenb.

Miconia ronliesneri Michelang.

Miconia roraimensis Ule

Miconia roseopetala Michelang.

Miconia rosmarinifolia (Griseb.) M. Gómez

Miconia rotundifolia (D. Don) Naudin

Miconia rubella (Raddi) R. Goldenb.

Miconia rubens (Sw.) Naudin

Miconia rubescens (Triana) Gamba \& Almeda

Miconia rubida (Cogn.) R. Goldenb.

Miconia rubiginosa (Bonpl.) DC.

Miconia rubra (Aubl.) Mabb.

Miconia rubricans Triana

Miconia rubrinervis (Naudin) Judd \& Bécquer

Miconia rubripetala Michelang.

Miconia rubripila (Cogn.) Judd \& Ionta

Miconia rubrisetulosa Ionta, Judd \& Skean

Miconia ruddae (Wurdack) Michelang.

Miconia rufa (Griseb.) Triana

Miconia rufescens (Aubl.) DC.

Miconia rufibarbis (Triana) Gamba \& Almeda

Miconia ruficalyx Gleason

Miconia ruficaulis Ionta \& Bécquer

Miconia rufinervis Judd

Miconia rufipila Triana

Miconia rufiramea Wurdack

Miconia rufistellata Judd \& Majure

Miconia rufoalpestris Skean, Judd \& Majure

Miconia rufostellulata Pittier

Miconia rugifolia Triana

Miconia rugosa Triana

Miconia ruizii Naudin

Miconia ruizteranii Wurdack

Miconia rupestris Ule

Miconia rupicola Gleason

Miconia rupticalyx Wurdack 
Miconia rusbyana Cogn.

Miconia ruschiana Caddah \& R. Goldenb.

Miconia russea Wurdack

Miconia rzedowskii de Santiago

Miconia sabiaensis (Brade) R. Goldenb.

Miconia sagotiana Cogn.

Miconia saldanhae Cogn.

Miconia salebrosa Wurdack

Miconia salicifolia Bonpl. ex Naudin

Miconia salicina (Ser. ex DC.) Mabb.

Miconia saltuensis Wurdack

Miconia samanensis Urb.

Miconia sancti-philippi Naudin

Miconia sandemanii Wurdack

Miconia sandwithii (Wurdack) Michelang.

Miconia sanguinea (D. Don) Triana

Miconia santanana Judd \& Skean

Miconia santaremensis Wurdack

Miconia santaritensis Almeda

Miconia santoslimae (Brade) R. Goldenb.

Miconia sarmentosa Cogn.

Miconia sastrei Wurdack

Miconia saulensis (Wurdack) Michelang.

Miconia savannarum R.O. Williams

Miconia saxatilis J.F. Macbr.

Miconia saxicola Brandegee

Miconia scaberrima Judd, Bécquer \& Majure

Miconia scabra Cogn.

Miconia scabrosa (L.) Ionta, Judd \& Skean

Miconia scalpta (Vent.) Ionta, Judd \& Skean

Miconia schippii Standl.

Miconia schlechtendalii Cogn.

Miconia schlimii Triana

Miconia schnellii Wurdack

Miconia schunkei Wurdack

Miconia schwackei Cogn.

Miconia sciaphila Judd \& Ionta

Miconia sciurea L. Uribe

Miconia sclerophylla Triana

Miconia scopulina (Brandegee) Michelang.

Miconia scutata Gleason

Miconia secuncaquetana Ocampo \& Almeda

Miconia secunda R.A. Howard \& E.A. Kellogg

Miconia secundiflora Cogn.

Miconia secundifolia Cogn.

Miconia secundivaricata Ocampo \& Almeda

Miconia secundo-angustifolia M. Gómez

Miconia secundo-lanceolata M. Gómez

Miconia secunfrancavillana Ocampo \& Almeda

Miconia secungrandifolia Ocampo \& Almeda

Miconia secunlongisepala Ocampo \& Almeda

Miconia secunmacdanielii Ocampo \& Almeda

Miconia secunmaguirei Ocampo \& Almeda

Miconia secunmexicana Ocampo \& Almeda

Miconia secunneblinensis Ocampo \& Almeda

Miconia secunpastazana Ocampo \& Almeda 
Miconia secunpetiolaris Ocampo \& Almeda Miconia secunretropila Ocampo \& Almeda Miconia secunrotundifolia Ocampo \& Almeda Miconia secunsanguinea Ocampo \& Almeda Miconia selleana Urb. \& Ekman

Miconia sellowiana Naudin

Miconia semijuga (Gleason) Michelang.

Miconia semisterilis Gleason

Miconia seposita Wurdack

Miconia septentrionalis Judd \& R.S. Beaman

Miconia septuplinervis Pittier

Miconia serialis DC.

Miconia sericea (D. Don) Michelang.

Miconia serpens (Triana) Michelang.

Miconia serrulata (DC.) Naudin

Miconia sessiliflora (Naudin) Michelang.

Miconia sessilifolia Naudin

Miconia sessilis Gamba \& Almeda

Miconia seticaulis Wurdack

Miconia setifera (Pilg.) Michelang.

Miconia setimarginata Pittier

Miconia setosa Wurdack

Miconia setosociliata Cogn.

Miconia setulosa Cogn.

Miconia shaferi Cogn.

Miconia shattuckii Standl.

Miconia shepherdii R. Goldenb. \& Reginato

Miconia siapensis (Wurdack) Michelang.

Miconia silicicola Gleason

Miconia silverstonei Wurdack

Miconia silvestris Gleason

Miconia silvicola (Gleason) Michelang.

Miconia silviphila Michelang.

Miconia simplex Triana

Miconia simplicicaulis (Naudin) R. Goldenb.

Miconia simpsonii (Wurdack) Michelang.

Miconia sintenisii Cogn.

Miconia skeaniana Judd

Miconia skutchiana Michelang.

Miconia smaragdina Naudin

Miconia smithii Cogn. ex Gleason

Miconia sneidernii Wurdack

Miconia sodiroi Wurdack

Miconia solearis (Naudin) Gamba \& Almeda

Miconia solenifera (Cogn.) Ocampo \& Almeda

Miconia solmsii Cogn.

Miconia sordida Triana

Miconia sororopana Michelang.

Miconia spadiciflora (Triana) Michelang.

Miconia spanantha Judd \& Majure

Miconia sparrei Wurdack

Miconia sparsisetulosa (Hoehne) R. Goldenb.

Miconia spatellophora Gleason

Miconia speciosa (A. St.-Hil. \& Naudin) Naudin

Miconia spectabilis (Gleason) Michelang.

Miconia spennerostachya Naudin 
Miconia sphaerocarpa (Cogn.) R. Goldenb.

Miconia sphagnicola Urb. \& Ekman

Miconia spicata (Gleason) Gamba \& Almeda

Miconia spicellata Bonpl. ex Naudin

Miconia spichigeri Wurdack

Miconia spinulidentata Cogn. \& Gleason

Miconia spinulosa Naudin

Miconia spireifolia Triana

Miconia splendens (Sw.) Griseb.

Miconia sprucei Triana

Miconia squamulosa (Sm.) Triana

Miconia staminea (Desr.) DC.

Miconia staphidioides (Naudin) Triana

Miconia steinbachii Markgr.

Miconia stelligera Cogn.

Miconia stellipilis Cogn.

Miconia stellulata Gleason

Miconia stellulitricha R. Goldenb. \& Michelang.

Miconia stenobotrys (Rich.) Naudin

Miconia stenocardia Cogn.

Miconia stenopetala Griseb.

Miconia stenophylla Wurdack

Miconia stenoptera (Gleason) Michelang.

Miconia stenostachya DC.

Miconia stenourea Triana

Miconia stephananthera Ule

Miconia stephanotricha (Naudin) Michelang.

Miconia stephentillettii Michelang.

Miconia sterilis Gleason

Miconia stevensiana Almeda

Miconia steyermarkii Gleason

Miconia stipitata Gleason

Miconia stipularis Naudin

Miconia striata (Vahl) Cogn.

Miconia strigilliflora (Naudin) R. Goldenb.

Miconia strigillosa (Sw.) Judd \& Ionta

Miconia strigosa (Triana) Wurdack

Miconia suaveolens Wurdack

Miconia subalpina Gleason

Miconia subandicola Wurdack

Miconia subciliata DC.

Miconia subcompressa Urb.

Miconia subcordata Cogn.

Miconia subcrustulata Beurl.

Miconia suberosa Meireles \& R. Goldenb.

Miconia subglabra Cogn.

Miconia subhirsuta (DC.) M. Gómez

Miconia sublanata (Cogn.) R. Goldenb.

Miconia submacrophylla Gleason

Miconia submontana (Rose ex Gleason) Michelang.

Miconia subnodosa Triana

Miconia subobruta (Wurdack) R. Goldenb. \& Michelang.

Miconia suborbicularis Cogn.

Miconia subpeltata (Kriebel \& Almeda) Almeda

Miconia subseriata (Naudin) R. Goldenb. \& Michelang.

Miconia subsessilifolia Wurdack 
Miconia subsimplex Pilg.

Miconia subspicata Wurdack

Miconia subtrinervis (Cogn.) R. Goldenb.

Miconia subulata (Gleason) R. Goldenb. \& Michelang.

Miconia subulipetala Wurdack

Miconia subvernicosa Cogn.

Miconia sulbahiensis (D'El Rei Souza) R. Goldenb.

Miconia sulcata J.F. Macbr.

Miconia sulcicaulis (Poepp. ex Naudin) Ocampo \& Almeda

Miconia sulfurea (Naudin) R. Goldenb.

Miconia summa Cuatrec.

Miconia superba Ule

Miconia superposita Wurdack

Miconia suprabasalis (R. Goldenb. \& Reginato) R. Goldenb.

Miconia susannae (Borhidi) Bécquer \& Michelang.

Miconia sylvatica (Schltdl.) Naudin

Miconia symphyandra Triana

Miconia symplectocaulos Pilg.

Miconia symplocoidea Triana

Miconia tabayensis Wurdack

Miconia tacanensis Wurdack

Miconia talamancensis Almeda

Miconia tamana Wurdack

Miconia taurina (Gleason) Michelang.

Miconia tenebrosa (Almeda) Almeda

Miconia tenensis Markgr.

Miconia tentaculicapitata Majure \& Judd

Miconia tentaculifera Naudin

Miconia tenuifolia (Donn. Sm.) Michelang.

Miconia tenuis Triana

Miconia teotepecensis de Santiago

Miconia tephrodes Wurdack

Miconia tepicana Standl.

Miconia tepuiensis (Wurdack) Michelang.

Miconia terborghii Wurdack

Miconia terera Naudin

Miconia ternata (Cogn.) R. Goldenb.

Miconia ternatifolia Triana

Miconia tetragona Cogn.

Miconia tetragonoloba (Cogn.) Judd, Bécquer \& Majure

Miconia tetramera (Urb. \& Ekman) Bécquer

Miconia tetrandra (Sw.) D. Don ex G. Don

Miconia tetrapetala (Almeda) Almeda

Miconia tetraptera (Cogn.) Ionta, Judd \& Skean

Miconia tetraquetra (Cham.) R. Goldenb.

Miconia tetrasperma Gleason

Miconia tetraspermoides Wurdack

Miconia tetrastoma Naudin

Miconia tetrazygioidea Bécquer \& Judd

Miconia tetrazygioides Urb. \& Ekman

Miconia thaminantha Wurdack

Miconia theaezans (Bonpl.) Cogn.

Miconia therezopolitana (Cogn.) R. Goldenb.

Miconia thomasiana DC.

Miconia thyrsiflora (D. Don) Naudin

Miconia thyrsoidea (D. Don ex DC.) Naudin 
Miconia thysanophylla Wurdack

Miconia tiliifolia Naudin

Miconia tillettii Wurdack

Miconia tinifolia Naudin

Miconia tiri Triana

Miconia titanophylla Gleason

Miconia tixixensis Standl. \& Steyerm.

Miconia tocachensis Michelang.

Miconia tocoaristata Michelang.

Miconia tocobovata Michelang.

Miconia tococa (Desr.) Michelang.

Miconia tococapitata Michelang.

Miconia tococaudata Michelang.

Miconia tocociliata Michelang.

Miconia tococinnamomea Michelang.

Miconia tococoidea (DC.) Michelang.

Miconia tococordata Michelang.

Miconia tococoronata Michelang.

Miconia tocohirta Michelang.

Miconia tocopauciflora Michelang.

Miconia tocoracemifera Michelang.

Miconia tomentosa (Rich.) D. Don ex DC.

Miconia tonduzii Cogn.

Miconia torbeciana (Urb. \& Ekman) Skean \& Judd

Miconia toroi Gleason

Miconia tovarensis Cogn.

Miconia traillii Cogn.

Miconia transversa Gleason

Miconia trauninensis (Cogn.) R. Goldenb.

Miconia trianae Cogn.

Miconia trianana Judd \& Bécquer

Miconia triangularis Gleason

Miconia triantha (E. Camargo \& R. Goldenb.) R. Goldenb.

Miconia tricaudata Wurdack

Miconia trichocalyx (Pittier) Michelang. \& Judd

Miconia trichogona J.F. Macbr.

Miconia trichophora Gleason

Miconia trichosantha (Almeda) Almeda

Miconia trichotoma (Desr.) DC.

Miconia tricostata (Urb. \& Ekman) Skean, Judd \& Majure

Miconia trimera Wurdack

Miconia trinervia (Sw.) D. Don ex Loudon

Miconia trinitensis (Crueg.) Michelang.

Miconia triplinervis Ruiz \& Pav.

Miconia tristis Spring

Miconia trujillensis Wurdack

Miconia truncata Triana

Miconia tschudyoides Cogn.

Miconia tuberculata (Naudin) Triana

Miconia tubulosa Gleason

Miconia tuckeri Gleason

Miconia tuerckheimii Cogn.

Miconia turbinata (Urb. \& Ekman) Skean \& Judd

Miconia turgida Gleason

Miconia turquinensis Urb. \& Ekman

Miconia ulei (Cogn.) R. Goldenb. 
Miconia uliginosa (Brade) R. Goldenb.

Miconia ulmarioides Naudin

Miconia umbellata (Mill.) Judd \& Ionta

Miconia umbriensis Wurdack

Miconia umbrosa Cogn.

Miconia undabunda (J.F. Macbr.) Michelang.

Miconia undata Triana

Miconia uninervis Alain

Miconia urbaniana Cogn.

Miconia urbanii (Cogn.) Judd

Miconia urceolata Urb.

Miconia uribei Wurdack

Miconia urophylla DC.

Miconia urticilamina Michelang.

Miconia urticoides Triana

Miconia utleyana (Almeda) Almeda

Miconia uvida Wurdack

Miconia uvifera Naudin

Miconia vaccinioides (Bonpl.) Naudin

Miconia valentinensis Bacci \& R. Goldenb.

Miconia valenzuelana Michelang. \& R.Goldenb.

Miconia valeriana (Standl.) Wurdack

Miconia valida Cogn.

Miconia vallartensis Zabalgoitia, Figueroa \& Muñiz-Castro

Miconia vallensis Wurdack

Miconia vallicola (Gleason) Michelang.

Miconia valtheri Naudin

Miconia vargasii Wurdack

Miconia variabilis Gamba \& Almeda

Miconia variifolia (Wurdack) Michelang.

Miconia vazquezii (Borhidi \& O. Muñiz) Ionta \& Bécquer

Miconia vegaensis (Cogn.) Judd, Bécquer \& Majure

Miconia velutina Triana

Miconia venosa (Gleason) Michelang.

Miconia ventricosa (Almeda) Almeda

Miconia venulosa Wurdack

Miconia veraguensis Gamba \& Almeda

Miconia verapazana Michelang.

Miconia verrucosa Cogn.

Miconia verruculosa (Wurdack) P.Jørg. \& C.Ulloa

Miconia versicolor Naudin

Miconia verticalis (N. Ruiz-R.) Michelang.

Miconia verticillifolia Michelang. \& R. Goldenb.

Miconia vesca Wurdack

Miconia vesiculosa (Cogn.) R. Goldenb.

Miconia vestita Almeda

Miconia victorinii Alain

Miconia vilhenensis Wurdack

Miconia villonacensis Wurdack

Miconia vincentina (Urb.) Judd \& Ionta

Miconia violacea Cogn.

Miconia violascens (Pilg.) Michelang. \& R. Goldenb.

Miconia virescens (Vahl) Triana

Miconia virgata (Sw.) Skean, Judd \& Majure

Miconia viridiflava (Brade) R. Goldenb.

Miconia viscidula Urb. \& Ekman 
Miconia viscosa (Cogn.) R. Goldenb.

Miconia vismioides Triana

Miconia vitiflora J.F. Macbr.

Miconia vittata (Linden \& André) Cogn.

Miconia volcanalis (Standl. \& Steyerm.) Michelang.

Miconia voronovii Gleason

Miconia vulcanicola (Donn. Sm.) Michelang.

Miconia vulcanidomatia Bécquer \& Skean

Miconia wagneri J.F. Macbr.

Miconia waltercampii Michelang.

Miconia walterjuddii Bécquer \& Michelang.

Miconia warmingiana Cogn.

Miconia weberbaueri Cogn.

Miconia weddellii Naudin

Miconia willdenowii Klotzsch ex Naudin

Miconia williamandersoniii Michelang.

Miconia wittii Ule

Miconia wolfei Wurdack

Miconia woodsii (Judd \& Skean) Ionta, Judd \& Skean

Miconia woytkowskii Wurdack

Miconia wrightii (Griseb.) Triana

Miconia wurdackii L. Uribe

Miconia xalapensis (Bonpl.) M. Gómez

Miconia xanthocoma (Naudin) R. Goldenb.

Miconia xantholasia (DC.) R. Goldenb.

Miconia xanthopogon (Naudin) R. Goldenb.

Miconia xanthostachya (Cogn.) R. Goldenb.

Miconia xenotricha Urb. \& Ekman

Miconia yamanigueyensis Bécquer \& Michelang.

Miconia yanachagaensis Michelang. \& R. Goldenb.

Miconia yatuensis Wurdack

Miconia yeseniae W. Palacios, D. Fernández \& Michelang.

Miconia yungasensis R. Goldenb. \& Michelang.

Miconia yunquensis Judd, Bécquer \& Majure

Miconia zamorensis Gleason

Miconia zanonii Judd, Skean \& R.S. Beaman

Miconia zarucchii Wurdack

Miconia zemurrayana Standl. \& L.O. Williams

Miconia zubenetana J.F. Macbr.

Microlicia abairana R. Romero \& Woodgyer

Microlicia acuminata Naudin

Microlicia agrestis Cogn.

Microlicia amblysepala Ule

Microlicia amplexicaulis Cogn.

Microlicia arenariaefolia DC.

Microlicia aurea Wurdack

Microlicia aureoglandulosa Woodgyer \& R. Romero

Microlicia avicularis Mart. ex Naudin

Microlicia baccharoides Mart. \& Schrank ex Naudin

Microlicia bahiensis Markgr.

Microlicia balsamifera Mart.

Microlicia baumgratziana A.B. Martins \& Koschn.

Microlicia benthamiana Triana

Microlicia blanchetiana Cogn.

Microlicia bradeana Hoehne

Microlicia caatingae J. Coelho \& R. Romero 
Microlicia canastrensis Naudin

Microlicia candolleana R. Romero \& Versiane

Microlicia capitata R.Pacifico, Almeda \& Fidanza

Microlicia catolensis Woodgyer \& Zappi

Microlicia cerifera (Gardner) A.B. Martins \& Almeda

Microlicia chrysantha Wurdack

Microlicia chrysoglandulosa R. Romero, Versiane, Fontelas \& D.O. Diniz-Neres

Microlicia ciliatoglandulosa R. Romero

Microlicia cogniauxiana R. Romero

Microlicia colombiana H. Mend. \& R. Romero

Microlicia comparilis Wurdack

Microlicia confertiflora Naudin

Microlicia consimilis Wurdack

Microlicia contasensis Woodgyer \& Zappi

Microlicia cordata (Spreng.) Cham.

Microlicia coriacea R.Pacifico, Almeda \& Fidanza

Microlicia crassa R. Romero

Microlicia crebropunctata Pilg.

Microlicia crenulata (DC.) Mart.

Microlicia crispa Woodgyer \& R. Romero

Microlicia cryptandra Naudin

Microlicia cuneata Naudin

Microlicia curralensis Brade

Microlicia curticalycina R. Romero \& Woodgyer

Microlicia cuspidifolia Mart. ex Naudin

Microlicia decipiens Naudin

Microlicia denudata Cogn.

Microlicia donii Fidanza \& R.Pacifico

Microlicia edmundoi Brade

Microlicia elegans Naudin

Microlicia ericoides D. Don

Microlicia euphorbioides Mart.

Microlicia fasciculata Mart.

Microlicia flava R. Romero

Microlicia flavovirens Woodgyer \& Zappi

Microlicia formosa Cham.

Microlicia frankii R.B. Pacifico \& Fidanza

Microlicia furnensis R. Romero

Microlicia ganevii Woodgyer \& R. Romero

Microlicia giuliettiana A.B. Martins \& Almeda

Microlicia glazioviana Cogn.

Microlicia graveolens DC.

Microlicia guanayana Wurdack

Microlicia harleyi Wurdack

Microlicia hatschbachii Wurdack

Microlicia helvola (Spreng) Triana

Microlicia hirta Pataro \& R. Romero

Microlicia hirticalyx R. Romero \& Woodgyer

Microlicia hirtoferruginea Naudin

Microlicia hispidula Naudin

Microlicia humilis Naudin

Microlicia inquinans Naudin

Microlicia insignis Cham.

Microlicia intercalycina Pataro \& R. Romero

Microlicia isophylla DC.

Microlicia isostemon Wurdack 
Microlicia jungermannioides DC.

Microlicia juniperina A. St.-Hil.

Microlicia karinae R. Pacifico\& Almeda

Microlicia latifolia D.O. Diniz-Neres \& M.J. Silva

Microlicia leucopetala Wurdack

Microlicia linifolia (DC.) Cham.

Microlicia longicalycina R. Romero

Microlicia longipedicellata Almeda \& A.B. Martins

Microlicia longirostrata R.Romero, Fontelas \& Versiane

Microlicia longisepala Wurdack

Microlicia luetzelburgii Markgr.

Microlicia lutea Markgr.

Microlicia macedoi L.B. Sm. \& Wurdack

Microlicia macropetala Pataro \& R. Romero

Microlicia macrophylla Naudin

Microlicia maculata R. Romero

Microlicia martiana O. Berg ex Triana

Microlicia maximowicziana Cogn.

Microlicia melanostagma Pilg.

Microlicia mendoncaei Cogn.

Microlicia microphylla Cogn.

Microlicia minima Markgr.

Microlicia minutiflora Cogn.

Microlicia monticola Wurdack

Microlicia morii Wurdack

Microlicia morrensis R. Pacifico \& Almeda

Microlicia mucugensis (Wurdack) Almeda \& A.B. Martins

Microlicia multicaulis Mart. ex Naudin

Microlicia mutabilis R.Pacifico, Almeda \& Fidanza

Microlicia myrtoidea Cham.

Microlicia naudiniana R. Romero

Microlicia neglecta Cogn.

Microlicia nervosa R. Romero

Microlicia noblickii (Wurdack) A.B. Martins \& Almeda

Microlicia nodotricha Almeda \&R.Pacifico

Microlicia nortecipoana R.B. Pacifico, Fidanza \& Almeda

Microlicia oblongifolia Naudin

Microlicia obovatifolia R.B. Pacifico, Fidanza \& Almeda

Microlicia obtusa Naudin

Microlicia obtusifolia Cogn. ex R. Romero

Microlicia oligochaeta Wurdack

Microlicia ordinata (Wurdack) Almeda \& A.B. Martins

Microlicia oxyanthera Naudin

Microlicia pabstii Brade

Microlicia pacificoi Almeda \& Fidanza

Microlicia parvula (Markgr.) Koschn. \& A.B. Martins

Microlicia passerina Naudin

Microlicia peruviana Cogn.

Microlicia petasensis Wurdack

Microlicia petiolulata Cogn.

Microlicia pilosissima Cogn.

Microlicia pinheiroi Wurdack

Microlicia piranii R.Pacifico, Almeda \& Fidanza

Microlicia plumosa Woodgyer \& Zappi

Microlicia polychaeta R.Pacifico, Almeda \& Fidanza

Microlicia polystemma Naudin 
Microlicia psammophila Wurdack

Microlicia pulchra Pataro \& R. Romero

Microlicia pusilla Cogn.

Microlicia ramosa Pilg.

Microlicia regeliana Cogn.

Microlicia reichardtiana Cogn.

Microlicia repanda R.Pacifico, Almeda \& Fidanza

Microlicia restingae R. Romero \& Woodgyer

Microlicia riedeliana Cogn.

Microlicia rotundifolia Ule

Microlicia rubra Ferreira-Alves \& R.Romero

Microlicia rugosa R. Romero \& Versiane

Microlicia sciophylla R.B. Pacifico \& Fidanza

Microlicia scoparia DC.

Microlicia semiriana Kosch. \& A.B.Martins

Microlicia serpyllifolia D. Don

Microlicia serrulata Cham.

Microlicia setosa DC.

Microlicia sickii Brade

Microlicia sincorensis Mart.

Microlicia sparsifolia R.Pacifico, Almeda \& Fidanza

Microlicia sphagnicola Gleason

Microlicia stenodonoides D.O. Diniz-Neres \& M.J. Silva

Microlicia stricta Cogn.

Microlicia subaequalis Wurdack

Microlicia subalata Wurdack

Microlicia suborbicularifolia Hoehne

Microlicia subsetosa DC.

Microlicia sulfurea Hoehne

Microlicia taxifolia Naudin

Microlicia tenuifolia R. Romero

Microlicia tetrasticha Cogn.

Microlicia tomentella Naudin

Microlicia torrendii Brade

Microlicia trembleyiformis Naudin

Microlicia trichocalycina DC.

Microlicia urceolata J. Coelho \& R. Romero

Microlicia veadeirana D.O.Diniz-Neres \& M. J. Silva

Microlicia vernicosa (A. Barreto ex Pedersoli) A.B. Martins \& Almeda

Microlicia vestita DC.

Microlicia viminalis Triana

Microlicia viscida R. Romero \& Versiane

Microlicia warmingiana Cogn.

Microlicia weddellii Naudin

Microlicia windischii Versiane, D.Nunes \& R.Romero

Microlicia woodii R. Pacifico, Almeda \& Fidanza

Microlicia wurdackiana Almeda \& A.B. Martins

Monochaetum alpestre Naudin

Monochaetum amabile Almeda

Monochaetum amistadense Almeda

Monochaetum bonplandii (Kunth) Naudin

Monochaetum brachyurum Naudin

Monochaetum calcaratum (DC.) Triana

Monochaetum candollei Cogn.

Monochaetum carbonoi Alvear \& Almeda

Monochaetum ciliatum Gleason 
Monochaetum cinereum Gleason

Monochaetum compactum Almeda

Monochaetum cordatum Almeda

Monochaetum deppeanum (Schltdl. \& Cham.) Naudin

Monochaetum dicranantherum Naudin

Monochaetum discolor H. Karst. ex Triana

Monochaetum ecaudatum Gleason

Monochaetum exaltatum Almeda

Monochaetum expansum (Gleason) Alvear \& Almeda

Monochaetum floribundum (Schltdl.) Naudin

Monochaetum glanduliferum Triana

Monochaetum gleasonianum Wurdack

Monochaetum hartwegianum Naudin

Monochaetum humboldtianum Walp.

Monochaetum laxifolium Gleason

Monochaetum lindeneanum Naudin

Monochaetum linearifolium Almeda

Monochaetum lineatum (D. Don) Naudin

Monochaetum longicaudatum Alvear \& Almeda

Monochaetum macrantherum Gleason

Monochaetum magdalenense Wurdack

Monochaetum mariae Wurdack

Monochaetum meridense Naudin

Monochaetum multiflorum (Bonpl.) Naudin

Monochaetum myrtoideum (Bonpl.) Naudin

Monochaetum neglectum Almeda

Monochaetum perijense Alvear \& Almeda

Monochaetum pulchrum Decne.

Monochaetum rodriguezii Wurdack

Monochaetum rotundifolium Cogn. ex Gleason

Monochaetum rubescens Gleason

Monochaetum stellulatum Naudin

Monochaetum strigosum Cogn.

Monochaetum subditivum J.F. Macbr.

Monochaetum subglabrum Gleason

Monochaetum tachirense Wurdack

Monochaetum talamancense Almeda

Monochaetum tenellum Naudin

Monochaetum tonaense Alvear \& Almeda

Monochaetum trichophyllum Almeda

Monochaetum uberrimum Sandwith

Monochaetum uribei Wurdack

Monochaetum vestitum Almeda, Al. Rodr. \& Garita

Monochaetum vulcanicum Cogn.

Monolena bracteata Gleason

Monolena cordifolia Triana

Monolena coriacea Triana

Monolena dressleri R.H. Warner

Monolena elliptica L. Uribe

Monolena grandiloba R.H. Warner

Monolena guatemalensis Donn. Sm.

Monolena lanceolata L. Uribe

Monolena morleyi R.H. Warner

Monolena multiflora R.H. Warner

Monolena ovata Cogn.

Monolena panamensis R.H. Warner 
Monolena pilosiuscula L. Uribe Monolena primuliflora Hook. f. Monolena pygmaea L. Uribe

Monolena trichopoda R.H. Warner

Mouriri acutiflora Naudin

Mouriri ambiconvexa Morley

Mouriri angulicosta Morley

Mouriri angustifolia Spruce ex Triana

Mouriri apiranga Spruce ex Triana

Mouriri arborea Gardner

Mouriri arenicola Morley

Mouriri bahiensis Morley

Mouriri barinensis (Morley) Morley

Mouriri brachyanthera Ducke

Mouriri brevipes Hook.

Mouriri cauliflora Mart. ex DC.

Mouriri cearensis Huber

Mouriri chamissoana Cogn.

Mouriri collocarpa Ducke

Mouriri colombiana Morley

Mouriri completens (Pittier) Burret

Mouriri crassifolia Sagot

Mouriri crassisepala Morley

Mouriri cyphocarpa Standl.

Mouriri densifoliata Ducke

Mouriri dimorphandra Morley

Mouriri domingensis (Tussac) Spach

Mouriri doriana Saldanha ex Cogn.

Mouriri duckeana Morley

Mouriri duckeanoides Morley

Mouriri dumetosa Cogn.

Mouriri elliptica Mart.

Mouriri emarginata Griseb.

Mouriri eugeniifolia Spruce ex Triana

Mouriri exadenia Morley

Mouriri exilis Gleason

Mouriri ficoides Morley

Mouriri floribunda Markgr.

Mouriri francavillana Cogn.

Mouriri froesii Morley

Mouriri gardneri Triana

Mouriri glazioviana Cogn.

Mouriri gleasoniana Standl.

Mouriri gonavensis Urb. \& Ekman

Mouriri grandiflora DC.

Mouriri guianensis Aubl.

Mouriri helleri Britton

Mouriri huberi Cogn.

Mouriri impressinerva Morley

Mouriri lancifolia Urb. \& Ekman

Mouriri latihila Morley

Mouriri laxiflora Morley

Mouriri longifolia (Kunth) Morley

Mouriri lunatanthera Morley

Mouriri megasperma Morley

Mouriri micradenia Ducke 
Mouriri micranthera Morley

Mouriri monopora Morley

Mouriri morleyi R. Goldenb. \& Meirelles

Mouriri muelleri Cogn.

Mouriri myrtifolia Spruce ex Triana

Mouriri myrtilloides (Sw.) Poir.

Mouriri nervosa Pilg.

Mouriri nigra (DC.) Morley

Mouriri obtusiloba Morley

Mouriri oligantha Pilg.

Mouriri osaensis Morley

Mouriri pachyphylla Burret

Mouriri panamensis Morley

Mouriri papillosa Morley

Mouriri pauciflora Spruce ex Cogn.

Mouriri peruviana Morley

Mouriri pranceana Morley

Mouriri pseudogeminata Pittier

Mouriri pusa Gardner ex Hook.

Mouriri regeliana Cogn.

Mouriri retentipetala Morley

Mouriri rhizophorifolia (DC.) Triana

Mouriri sagotiana Triana

Mouriri sellowiana (O. Berg) Burret

Mouriri sideroxylon Sagot ex Triana

Mouriri spathulata Griseb.

Mouriri spruceana Morley

Mouriri steyermarkii Standl.

Mouriri subumbellata Triana

Mouriri tessmannii Markgr.

Mouriri torquata Morley

Mouriri trunciflora Ducke

Mouriri tuberculata Morley \& K. Thomsen

Mouriri uncitheca Morley \& Wurdack

Mouriri valenzuelana A. Rich.

Mouriri vernicosa Naudin

Mouriri viridicosta Morley

Neblinanthera cumbrensis Wurdack

Nepsera aquatica (Aubl.) Naudin

Noterophila bivalvis (Aubl.) Kriebel \& M.J.R.Rocha

Noterophila crassipes (Naudin) Kriebel \& M.J.R.Rocha

Noterophila genliseoides (Hoehne) Kriebel \& M.J.R.Rocha

Noterophila limnobios (DC.) Mart.

Noterophila nana (Ule) Kriebel \& M.J.R.Rocha

Noterophila rosulans (Huber) Kriebel \& M.J.R.Rocha

Ochthephilus repentinus Wurdack

Opisthocentra clidemioides Benth. \& Hook. f.

Pachyloma coriaceum DC.

Pachyloma huberioides (Naudin) Triana

Pachyloma pusillum Wurdack

Pachyloma setosum Wurdack

Phainantha laxiflora (Triana) Gleason

Phainantha maguirei Wurdack

Phainantha myrteoloides Wurdack

Phainantha shuariorum C. Ulloa \& D.A. Neill

Phainantha steyermarkii Wurdack 
Physeterostemon aonae Amorim, Michelang. \& R. Goldenb.

Physeterostemon fiaschii R. Goldenb. \& Amorim

Physeterostemon gomesii Amorim \& R. Goldenb.

Physeterostemon jardimii R. Goldenb. \& Amorim

Physeterostemon thomasii Amorim, Michelang. \& R. Goldenb.

Pilocosta campanensis (Almeda \& Whiffin) Almeda

Pilocosta erythrophylla (Gleason) Almeda \& Whiffin

Pilocosta nana (Standl.) Almeda \& Whiffin

Pilocosta nubicola Almeda

Pilocosta oerstedii (Triana) Almeda \& Whiffin

Pleroma ackermannii (Cogn.) P. J. F. Guim. \& Michelang.

Pleroma ademarii (P.J.F.Guim., R.Romero \& Leoni) P. J. F. Guim. \& Michelang.

Pleroma aemulum (Schrank et Mart ex DC.) Triana

Pleroma alatum (Cogn.) P. J. F. Guim. \& Michelang.

Pleroma amoenum (Herzog) P. J. F. Guim. \& Michelang.

Pleroma andersregnellii P.J.F.Guim. \& Michelang.

Pleroma angustifolium (Naud.) Triana

Pleroma apparicioi (Brade) P. J. F. Guim. \& Michelang.

Pleroma arboreum Gardner

Pleroma arenarium (Cogn.) P. J. F. Guim. \& Michelang.

Pleroma asperius (Cham.) Triana

Pleroma australe Triana

Pleroma axillare (Cogn.) P. J. F. Guim. \& Michelang.

Pleroma bahiense (Wurdack) P. J. F. Guim. \& Michelang.

Pleroma bandeirae P.J.F. Guim. \& Michelang.

Pleroma barnebyanum (Wurdack) P. J. F. Guim. \& Michelang.

Pleroma benthamianum Gardner

Pleroma bergianum (Cogn.) P. J. F. Guim. \& Michelang.

Pleroma blanchetianum (Cogn.) P. J. F. Guim. \& Michelang.

Pleroma boraceiensis (Brade) P. J. F. Guim. \& Michelang.

Pleroma boudetii (P.J.F.Guim. \& R.Goldenb.) P. J. F. Guim. \& Michelang.

Pleroma bracteolatum (J. G. Freitas, A. K. A.Santos \& R. P.

Pleroma caissarum F.S. Mey.

Pleroma candolleanum (Mart. ex. DC.) Triana

Pleroma canescens (D.Don) P. J. F. Guim. \& Michelang.

Pleroma carajasense K. Rocha, R. Goldenb. \& F.S. Mey.

Pleroma cardinale (Bonpl.) Triana

Pleroma carvalhoi (Wurdack) P. J. F. Guim. \& Michelang.

Pleroma castellense (Brade) P. J. F. Guim. \& Michelang.

Pleroma cecilianum P.J.F.Guim. \& Oliveira da Silva

Pleroma cinereum (Cogn.) P. J. F. Guim. \& Michelang.

Pleroma claussenii (Naud.) Triana

Pleroma clavatum (Persoon) P. J. F. Guim. \& Michelang.

Pleroma cleistoflorum (Ule) P.J.F.Guim., Oliveira da Silva \& Michelang.

Pleroma clidemioides Berg. ex Trianna

Pleroma collinum (Naudin) Triana

Pleroma comosum (J. G. Freitas, A. K. A.Santos \& R. P.

Pleroma cordifolium (Cogn.) P. J. F. Guim. \& Michelang.

Pleroma costatocalyx F.S. Mey., L. Kollmann \& R. Goldenb.

Pleroma crassirame (Cogn.) P. J. F. Guim. \& Michelang.

Pleroma cristatum (Brade) P. J. F. Guim. \& Michelang.

Pleroma cryptadenum (Gleason) P. J. F. Guim. \& Michelang.

Pleroma cucullatum F.S. Mey., Fraga \& R. Goldenb.

Pleroma decemcostatum (Cogn.) P. J. F. Guim. \& Michelang.

Pleroma dendroides (Naud.) Triana

Pleroma discolor (Brade) P. J. F. Guim. \& Michelang. 
Pleroma divaricatum (Cogn.) P. J. F. Guim. \& Michelang. Pleroma dubium (Cham) P. J. F. Guim. \& Michelang. Pleroma dusenii (Cogn.) P. J. F. Guim. \& Michelang. Pleroma echinatum Gardner Pleroma edmundoi (Brade \& Markgraf) P.J.F. Guim. \& Michelang. Pleroma eichleri (Cogn.) P. J. F. Guim. \& Michelang. Pleroma elegans Gardner Pleroma estrellense (Raddi) P.J.F.Guim. \& Michelang. Pleroma ferricolum A.L.F.Oliveira, R.Romero \& P.J.F. Guim. Pleroma fissinervium (Schrank \& Mart. ex DC.) Gardner Pleroma floribundum (Cogn.) P. J. F. Guim. \& Michelang. Pleroma fontanae F.S. Mey., L. Kollmann \& R. Goldenb. Pleroma formosum (Cogn.) P. J. F. Guim. \& Michelang. Pleroma fornograndense F.S. Mey., R. Goldenb. \& L. Kollmann Pleroma fothergillae (Schrank et Mat. ex DC.) Triana Pleroma foveolatum (Naud.) Triana Pleroma fragae L. Kollmann \& R. Goldenb. Pleroma francavillanum (Cogn.) P. J. F. Guim. \& Michelang. Pleroma frigidulum (Schrank et Mart. ex DC.) Triana Pleroma gardneri (Naud.) P. J. F. Guim. \& Michelang. Pleroma gaudichaudianum (DC.) A. Gray Pleroma gertii P.J.F. Guim. \& Michelang. Pleroma glutinosum (Markgr.) P. J. F. Guim. \& Michelang. Pleroma goldenbergii (F.S. Mey., P.J.F. Guim. \& Kozera) P. J. F. Guim. \& Michelang. Pleroma granulosum (Desr.) D. Don Pleroma guartelaense F.S.Mey. \& R.Goldenb.

Pleroma hatschbachii (Wurdack) P. J. F. Guim. \& Michelang. Pleroma heteromallum (D.Don) D.Don Pleroma hirsutissimum (Cogn.) P. J. F. Guim. \& Michelang. Pleroma hospitum (Schrank et Mart. ex DC.) Triana Pleroma integerrima (R.Romero \& A.B.Martins) P.J.F. Guim. \& Michelang. Pleroma itatiaiae (Wawra) P. J. F. Guim. \& Michelang. Pleroma kleinii (Wurdack) P. J. F. Guim. \& Michelang. Pleroma kollmannianum F.S.Mey. \& R.Goldenb. Pleroma kuhlmannii (Brade) P. J. F. Guim. \& Michelang. Pleroma laevicaule (Cogn. ex Wurdack) P. J. F. Guim. \& Michelang. Pleroma langsdorffianum (Bonpl.) Triana Pleroma leopodinense L.Kollmann \& R.Goldenb. Pleroma lhotzkyana (C. Presl.) Triana Pleroma lilacinum (Cogn.) P. J. F. Guim. \& Michelang. Pleroma limae (Brade) P. J. F. Guim. \& Michelang. Pleroma lithophilum (Wurdack) P. J. F. Guim. \& Michelang. Pleroma luetzelburgii (Markgr.) P. J. F. Guim. \& Michelang. Pleroma lutzii (Brade) P. J. F. Guim. \& Michelang.

Pleroma macrochiton (Mart. ex DC.) Triana Pleroma manicatum (Cogn.) P. J. F. Guim. \& Michelang. Pleroma marinarum P.J.F.Guim. \& Fraga

Pleroma martiale (Cham.) Triana

Pleroma martiusianum (DC.) P. J. F. Guim. \& Michelang. Pleroma marumbiense (Wurdack) P. J. F. Guim. \& Michelang. Pleroma maximilianum (DC.) Triana Pleroma melanocalyx (R.Romero, P.J.F.Guim. \& Leoni) P. J. F. Guim. \& Michelang. Pleroma mellobarretoi (Brade) P. J. F. Guim. \& Michelang. Pleroma michelangelii P.J.F. Guim. \& J.G. Freitas Pleroma microphyllum (Cogn. ex Schwacke) P. J. F. Guim. \& Michelang. Pleroma minus (R.Romero \& A.B.Martins) P.J.F. Guim. \& Michelang. 
Pleroma minutiflorum (Cogn.) P. J. F. Guim. \& Michelang. Pleroma mirabile (Brade \& Markgr.) P. J. F. Guim. \& Michelang. Pleroma molle (Cham.) Triana Pleroma mosenii (Cogn.) P. J. F. Guim. \& Michelang. Pleroma mourae (Cogn.) P. J. F. Guim. \& Michelang. Pleroma mutabile (Vell.) Triana Pleroma noblickii (Wurdack) P. J. F. Guim. \& Michelang. Pleroma nodosum (Wurdack ex Cogn.) P. J. F. Guim. \& Michelang. Pleroma ochypetala (Ruiz et Pavon) D. Don Pleroma oleifolium (DC.) R. Romero \& Versiane Pleroma oreophilum (Wurdack) P. J. F. Guim. \& Michelang. Pleroma pallidum (Cogn.) P. J. F. Guim. \& Michelang. Pleroma pauloalvinii (Vinha) P. J. F. Guim. \& Michelang. Pleroma penduliflorum Fraga \& P.J.F.Guim.

Pleroma pereirae (Brade \& Markgr.) P. J. F. Guim. \& Michelang. Pleroma petiolatum (R.Romero \& A.B.Martins) P.J.F. Guim. \& Michelang. Pleroma pilosum (Cogn.) P. J. F. Guim. \& Michelang. Pleroma quartzophilum (Brade) P. J. F. Guim. \& Michelang. Pleroma raddianum (DC.) Gardner Pleroma radula (Markgr.) P. J. F. Guim. \& Michelang. Pleroma ramboi (Brade) P. J. F. Guim. \& Michelang. Pleroma redivivum O.Berg ex Triana Pleroma regelianum (Cogn.) P. J. F. Guim. \& Michelang. Pleroma reitzii (Brade) P. J. F. Guim. \& Michelang. Pleroma riedelianum (Cogn.) P. J. F. Guim. \& Michelang. Pleroma rigidulum ((Naudin) Wurdack) P. J. F. Guim. \& Michelang. Pleroma riparium (Markgr.) P. J. F. Guim. \& Michelang. Pleroma robustm (Cogn.) P. J. F. Guim. \& Michelang. Pleroma rubrobracteatum (R.Romero \& P.J.F.Guim.) P. J. F. Guim. \& Michelang. Pleroma rubrum J.G.Freitas

Pleroma rupicola (Hoehne) P. J. F. Guim. \& Michelang. Pleroma salviaefolium (Cham.) Triana Pleroma scaberrimum Triana Pleroma schenckii (Cogn.) P. J. F. Guim. \& Michelang. Pleroma schwackei (Cogn.) P. J. F. Guim. \& Michelang. Pleroma sellowianum (Cham) P. J. F. Guim. \& Michelang. Pleroma semidecandrum (Schrank et Mart. ex DC.) Triana Pleroma setosociliatum (Cogn.) F.S.Mey. \& F.B.Matos Pleroma stellipile (Wurdack) P. J. F. Guim. \& Michelang. Pleroma stenocarpa (Schrank et Mart. ex DC.) Triana Pleroma stipulaceum (Guimarães da Vinha) P. J. F. Guim. \& Michelang. Pleroma subglabrum (Wurdack) P. J. F. Guim. \& Michelang. Pleroma subsessilis F.S. Mey. \& L. Kollmann Pleroma taperoense (Wurdack) P. J. F. Guim. \& Michelang. Pleroma tedescoi (Meirelles, L.Kollmann \& R.Goldenb.) P.J.F. Guim. \& Michelang. Pleroma thereminianum (DC.) Triana Pleroma tomentulosum (Wurdack) P. J. F. Guim. \& Michelang.

Pleroma trichopodum DC.

Pleroma trinervium P.J.F. Guim.

Pleroma tuberosum Gardner ex Triana

Pleroma urceolare (Schrank \& Mart. ex DC.) Triana

Pleroma ursinum (Cham.) Triana

Pleroma urvilleanum (DC.) P.J.F.Guim. \& Michelang.

Pleroma velutinum (Naud.) Triana

Pleroma venetiense F.S. Mey., L. Kollmann \& R. Goldenb.

Pleroma villosissimum Mart. ex.Triana 
Pleroma vimineum (D. Don) D. Don

Pleroma virgatum Gardner

Pleroma wurdackianum (R.Romero \& A.B.Martins) P.J.F. Guim. \& Michelang.

Poteranthera annectans Wurdack

Poteranthera leptalea (Almeda) M. J. Rocha, P.J.F. Guim. \& R. Romero

Poteranthera pusilla Bong.

Poteranthera warmingii (Cogn.) Almeda \& R.Pacifico

Poteranthera windischii Kriebel

Pseudoernestia cordifolia (O.Berg ex Triana) Krasser

Pseudoernestia glandulosa (Gleason) M.J.R.Rocha \& P.J.F.Guim.

Pterogastra divaricata (Bonpl.) Naudin

Pterogastra major Triana

Pterogastra minor Naudin

Pterolepis alpestris Triana

Pterolepis buraeavii Cogn.

Pterolepis cataphracta (Cham.) Triana

Pterolepis cearensis Huber

Pterolepis glomerata (Rottb.) Miq.

Pterolepis haplostemona Almeda \& A.B. Martins

Pterolepis parnassiifolia (DC.) Triana

Pterolepis perpusilla Cogn.

Pterolepis piatensis (S.S. Renner) J.G. Freitas \& A.K.A. Santos

Pterolepis picorondonica S.S. Renner

Pterolepis polygonoides Triana

Pterolepis repanda (DC.) Triana

Pterolepis riedeliana Cogn.

Pterolepis rotundifolia Wurdack

Pterolepis stenophylla Gleason

Pterolepis trichotoma (Rottb.) Cogn.

Quipuanthus epipetricus Michelang. \& C. Ulloa

Rhexia alifanus Walter

Rhexia aristosa Britton

Rhexia cubensis Griseb.

Rhexia interior Pennell

Rhexia lutea Walter

Rhexia mariana L.

Rhexia nashii Small

Rhexia nuttallii C.W. James

Rhexia parviflora Chapm.

Rhexia petiolata Walter

Rhexia salicifolia Kral \& Bostick

Rhexia ventricosa Fernald \& Griscom

Rhexia virginica L.

Rhynchanthera acuminata Benth.

Rhynchanthera apurensis Wurdack

Rhynchanthera brachyrhyncha Cham.

Rhynchanthera bracteata Triana

Rhynchanthera cordata DC.

Rhynchanthera dichotoma (Desr.) DC.

Rhynchanthera gardneri Naudin

Rhynchanthera grandiflora (Aubl.) DC.

Rhynchanthera hassleriana Kraenzl.

Rhynchanthera hispida Naudin

Rhynchanthera latifolia Cogn.

Rhynchanthera mexicana DC.

Rhynchanthera novemnervia DC. 
Rhynchanthera paludicola (Donn. Sm.) Gleason

Rhynchanthera rosea Cogn.

Rhynchanthera secundiflora Naudin

Rhynchanthera serrulata (Rich.) DC.

Rhynchanthera ternata Cogn.

Rhynchanthera ursina Naudin

Rhynchanthera verbenoides Cham.

Rostranthera tetraptera (Cogn.) M.J.R.Rocha \& P.J.F.Guim.

Rupestrea carvalhoana (Baumgratz \& D'El Rei Souza) Almeda, Michelang. \& R. Goldenb.

Rupestrea johnwurdakiana (Baumgratz \& D'El Rei Souza) Michelang., Almeda \& R. Goldenb.

Salpinga ciliata Pilg.

Salpinga dimorpha (Gleason) Wurdack

Salpinga glandulosa (Gleason) Wurdack

Salpinga longifolia (Cham.) Triana

Salpinga maguirei Gleason

Salpinga maranonensis Wurdack

Salpinga paleacea Wurdack

Salpinga peruviana (Cogn.) Wurdack

Salpinga pusilla (Gleason) Wurdack

Salpinga secunda Schrank \& Mart. ex DC.

Sandemania hoehnei (Cogn.) Wurdack

Schwackaea cupheoides (Benth.) Cogn.

Siphanthera arenaria (DC.) Cogn.

Siphanthera cordata Pohl ex DC.

Siphanthera cordifolia (Benth.) Gleason

Siphanthera cowanii Wurdack

Siphanthera dawsonii Wurdack

Siphanthera duidae (Gleason) Wurdack

Siphanthera fasciculata (Gleason) Almeda \& O.R. Rob.

Siphanthera foliosa (Naudin) Wurdack

Siphanthera gracillima (Naudin) Wurdack

Siphanthera hostmannii Cogn.

Siphanthera paludosa (DC.) Cogn.

Siphanthera subtilis Pohl ex DC.

Siphanthera todziae Almeda \& O.R. Rob.

Siphanthera vaupesana Wurdack

Siphanthera wurdakii Almeda \& O.R. Rob.

Stanmarkia medialis (Standl. \& Steyerm.) Almeda

Stanmarkia spectabilis Almeda

Stenodon gracilis O. Berg ex Triana

Stenodon suberosus Naudin

Tateanthus duidae Gleason

Tessmannianthus calcaratus (Gleason) Wurdack

Tessmannianthus carinatus Almeda

Tessmannianthus cenepensis Wurdack

Tessmannianthus cereifolius Almeda

Tessmannianthus gordonii Almeda

Tessmannianthus heterostemon Markgr.

Tessmannianthus quadridomius Wurdack

Tibouchina aegopogon (Naud.) Cogn.

Tibouchina alata (Wurdack ex Cogn.) P.J.F. Guim. \& Michelang.

Tibouchina albescens Cogn. ex P.J.F. Guim., A.L.F. Oliveira \& R. Romero

Tibouchina araguaiensis P.J.F.Guim.

Tibouchina aspera Aubl.

Tibouchina barbigera (Naudin) Baill.

Tibouchina bicolor (Naudin) Cogn. 
Tibouchina bipenicillata (Naudin) Cogn.

Tibouchina brevisepala Cogn.

Tibouchina bruniana P.J.F.Guim.

Tibouchina caatingae J.G. Freitas

Tibouchina calycina Cogn.

Tibouchina catharinae Pittier

Tibouchina cubense (A.Rich.) M.Gómez

Tibouchina cujabensis Cogn.

Tibouchina dissitiflora Wurdack

Tibouchina duidae Gleason

Tibouchina edmundoi Brade

Tibouchina exasperata (Naudin) Cogn.

Tibouchina fraterna N.E.Br.

Tibouchina fraterna subsp. paruana Wurdack

Tibouchina huberi Wurdack

Tibouchina johnwurdackiana Todzia

Tibouchina karstenii Cogn.

Tibouchina llanorum Wurdack

Tibouchina mathaei Cogn.

Tibouchina melastomoides (Naudin) Cogn.

Tibouchina nigricans Cogn. ex P.J.F.Guim., A.L.F. Oliveira \& R.Romero

Tibouchina octopetala Cogn. ex Britton

Tibouchina papyrus (Pohl) Toledo

Tibouchina pogonanthera (Naudin) Cogn .

Tibouchina rosanae P.J.F.Guim. \& Woodgyer

Tibouchina sickii Brade

Tibouchina sipapoana Gleason

Tibouchina spruceana Cogn.

Tibouchina steyermarkii Wurdack

Tibouchina striphnocalyx (DC.) Pittier

Tibouchina verticillaris Cogn.

Tibouchina xochiatencana de Santiago

Trembleya acuminata R.Pacifico \& Fidanza

Trembleya altoparaisensis R. Pacifico, Almeda \& Fidanza

Trembleya calycina Cham.

Trembleya chamissoana Naudin ex Cogn.

Trembleya elegans (Cogn.) Almeda \& A.B. Martins

Trembleya hatschbachii Wurdack \& E.Martins

Trembleya laniflora (D. Don) Cogn.

Trembleya neopyrenaica Naudin

Trembleya parviflora (D. Don) Cogn.

Trembleya pentagona Naudin

Trembleya phlogiformis DC.

Trembleya pithyoides Cham.

Trembleya pradosiana Netto

Trembleya rosmarinoides DC.

Trembleya thomazii R.B. Pacifico \& Fidanza

Trembleya tridentata Naudin

Triolena agrimonioides (Triana) Almeda \& Alvear

Triolena allardii (Wurdack) Wurdack

Triolena amazonica (Pilg.) Wurdack

Triolena asplundii Wurdack

Triolena auriculata (Triana) Almeda \& Alvear

Triolena barbeyana Cogn.

Triolena calciphila (Standl. \& Steyerm.) Standl. \& L.O. Williams

Triolena campii (Wurdack) Wurdack 
Triolena dressleri Wurdack

Triolena hirsuta (Benth.) Triana

Triolena hygrophylla (Naudin) L.O. Williams

Triolena izabalensis Standl. \& Steyerm.

Triolena lanceolata (Gleason) Almeda \& Alvear

Triolena obliqua (Triana) Wurdack

Triolena paleacea (Triana) Almeda \& Alvear

Triolena paleolata Donn. Sm.

Triolena pedemontana Wurdack

Triolena pileoides (Triana) Wurdack

Triolena pluvialis (Wurdack) Wurdack

Triolena pumila Umaña \& Almeda

Triolena purpurea (Gleason) Almeda \& Alvear

Triolena pustulata Triana

Triolena rojasae Michelang. \& R. Goldenb.

Triolena scorpioides Naudin

Triolena spicata (Triana) L.O. Williams

Triolena stenophylla (Standl. \& Steyerm.) Standl. \& L.O. Williams

Triolena vasquezii Michelang. \& R.Goldenb.

Tryssophyton merumense Wurdack

Tryssophyton quadrifolius K.Wurdack \& Michelang.

Votomita cupuliformis Morley \& Almeda

Votomita guianensis Aubl.

Votomita monadelpha (Ducke) Morley

Votomita monantha (Urb.) Morley

Votomita orbinaxia Morley

Votomita orinocensis Morley

Votomita plerocarpa (Morley) Morley

Votomita pubescens Morley

Votomita roraimensis Morley

Votomita ventuarensis Morley

Wurdastom bullata (Wurdack) B. Walln.

Wurdastom cuatrecasasii (Wurdack) B. Walln.

Wurdastom dorrii (Wurdack) B. Walln.

Wurdastom dudleyi (Wurdack) B. Walln.

Wurdastom ecuadorense (Wurdack) B. Walln.

Wurdastom hexamera (Wurdack) B. Walln.

Wurdastom lateriflora Humberto Mend.

Wurdastom sneidernii (Wurdack) B. Walln.

Wurdastom subglabra (Wurdack) B. Walln. 